Summer 2019

\title{
A theory of constitutive tropes
}

Anthony Parisi

University of lowa

Follow this and additional works at: https://ir.uiowa.edu/etd

Part of the Philosophy Commons

Copyright ( 2019 Anthony Salvatore Parisi

This dissertation is available at lowa Research Online: https://ir.uiowa.edu/etd/7009

\section{Recommended Citation}

Parisi, Anthony. "A theory of constitutive tropes." PhD (Doctor of Philosophy) thesis, University of lowa, 2019.

https://doi.org/10.17077/etd.tvc3-ly02

Follow this and additional works at: https://ir.uiowa.edu/etd

Part of the Philosophy Commons 


\section{A THEORY OF CONSTITUTIVE TROPES}

by

Anthony Parisi

A thesis submitted in partial fulfillment

of the requirements for the Doctor of Philosophy

degree in Philosophy in the

Graduate College of

The University of Iowa

August 2019

Thesis Supervisor: Professor Evan Fales 
Copyright by

Anthony Parisi

August 2019

All Rights Reserved 


\section{Acknowledgements}

I would like to thank my committee for supporting me and helping me through this process, along with a special thanks to my thesis supervisor Evan Fales, without whom this project would not be what it is today. His help through being an interlocutor and an honest opponent in many cases was vital to the production of this work. I would also like to thank

my partner, Dr. Jane Rosen, for her continuous help and support, as well as my family and my many friends for their support in what has been at times a rough and difficult process. 


\begin{abstract}
The purpose of this work is to provide a metaphysical theory of properties and scientific laws. This sentence will require some unpacking. By a 'metaphysical' theory here, I mean a theory of what exists in the world. In this investigation I am primarily concerned with a theory of what properties there are in the world and the role they play in scientific laws. This may be contrasted with a linguistic or epistemic project, as it is not primarily about our language, ideas, or theorizing but rather about what is in the world itself. Properties are what we may pre-philosophically think of as the characteristics of an object: such as its height, weight, color, etc. Investigation may cause us to doubt whether some of these pre-philosophical properties are genuine in a metaphysical sense: whether or not the property is actually present as a feature of the world. By scientific laws, I mean the statements we make in the form of exceptionless generalities about the world within the sciences. Here I do not mean to evaluate how science comes about these generalizations, only that they do and how these generalizations may be grounded in a metaphysical theory of properties. The link between these two things: properties and scientific laws, comes about because our scientific laws prominently feature properties: velocity, temperature, charge, viscosity, etc.
\end{abstract}

After a review of some of the theories currently proposed in the philosophical literature along with a treatment of some of the problems that arise out of these theories, I will propose a new theory. This theory, entitled 'Constitutive Trope Theory' is a form of a 'bundle trope theory' as it proposes that objects in the world are composed entirely out of particularized properties. However, rather than proposing a primitive relation that does the bundling, this theory will propose that, at least for the objects we are familiar with, 
properties arise out of relations between lower-level properties that instantiate them. For example, a mammal may exist because of the relationship between the organs that lead to its unique properties as a mammal. Those organs in turn exist because of relationships between their cells that lead to their unique properties as a particular kind of organ. And so on down the chain until we hit 'foundational properties': properties which are not dependent on any other properties for their existence. I will say very little about these properties as I do not believe that any such properties have currently been found and will argue that such theorizing is premature before such properties have been empirically identified. Following this account, I will consider some of the problems that must be overcome and some final considerations in favor of this theory over other competing theories of properties and scientific laws. 


\section{Public Abstract}

The purpose of this work is to provide an explanation of properties and scientific laws. The key goal is to understand what there is in the world in terms of objects and their properties, and what it is about the world that makes scientific laws true. I start with reviewing some prior attempts to provide such explanations and showing some of their inadequacies before proposing a new explanation. This explanation, entitled 'Constitutive Trope Theory' says that objects are composed of their properties, but reliant on lower-level objects for their existence. For instance, a water of molecule will be identical to the properties it possesses, but it will have those properties in virtue of the atoms and bonds that form that particular water molecule. These properties are thought of as being "powerful properties," as they respond to the presence of other properties and conditions in particular ways. That these properties are powerful properties is then taken to be the basis of scientific laws, as these powers can be described in terms of laws. 


\section{Table of Contents}

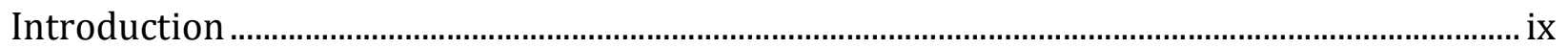

Chapter 1: Motivation, Method, and Desiderata ............................................................................ 1

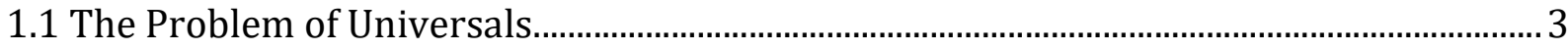

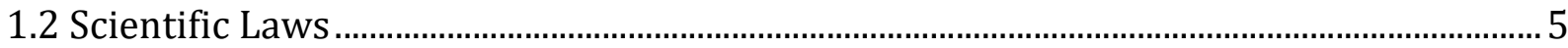

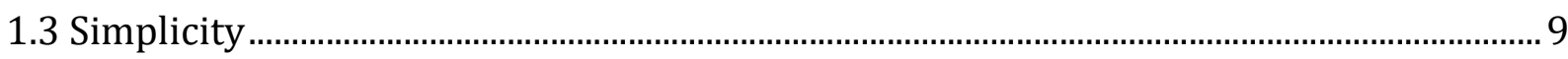

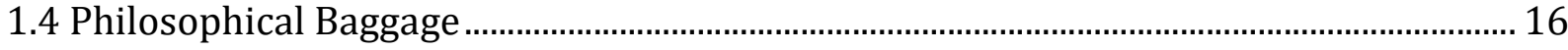

1.5 Desiderata of an Account.................................................................................................... 20

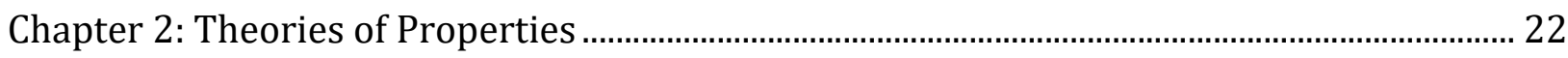

2. 1 Realist Theories ............................................................................................................... 24

2.1.1 Armstrong's Theory of Universals .............................................................................. 24

2.1.2 Other Realisms ……………………………………………………………………….... 31

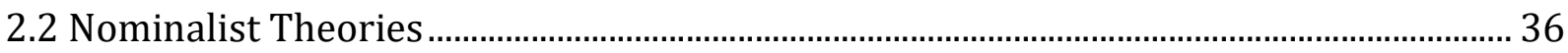

2.2.1 Lewisian Objects and Possible Worlds ....................................................................... 38

2.2.2 Problems of Nominalism ................................................................................................ 45

2.3 Trope Theories ............................................................................................................... 50

2.3.1 Campbell's Bundle Theory …………………………................................................... 50

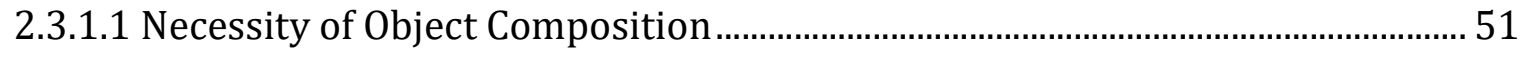

2.3.1.2 Resemblance and the Trope Theory …………………………………………..... 53

2.3.1.3 Scientific Laws and the Bundle Theory …………………………………………. 56

2.3.1.4 Field Theory of Tropes ......................................................................................... 59

2.3.2 Martin's Substratum Theory …………………………………………………………... 64

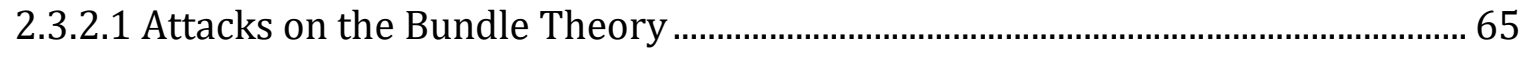

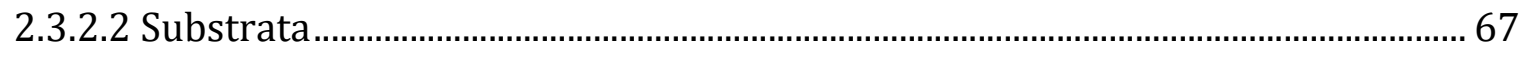

2.3.2.3 Resemblance for Martin........................................................................................ 70

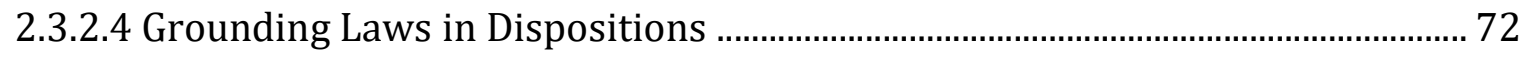

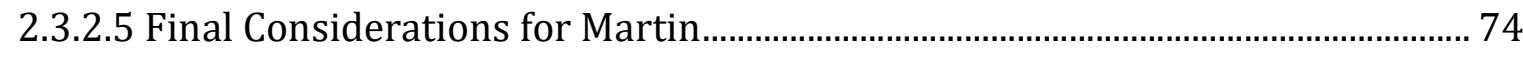

2.3.3 Conclusions for Theories of Properties ........................................................................ 76 
Chapter 3: Constitutive Trope Theory ………………….......................................................... 79

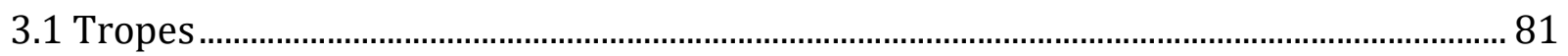

3.1.1 A Theory of Constitutive Parts ..................................................................................... 81

3.1.2 Aggregates and Emergent Tropes.................................................................................. 88

3.1.3 Eliminable Properties .................................................................................................... 91

3.1.4 Determinables and Determinants...................................................................................... 94

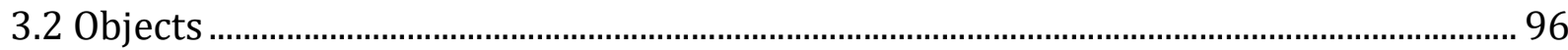

3.2.1 Change and Persistence .............................................................................................. 97

3.2.2 Identity of Objects ....................................................................................................... 98

3.2.3 Pseudo-objects ........................................................................................................101

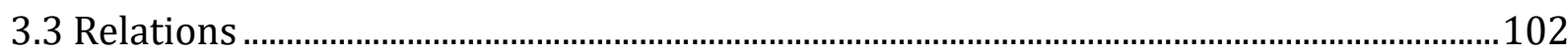

3.3.1 Relata ....................................................................................................................105

3.3.2 Compresence Relations and Objects.........................................................................108

3.4 The Problem of Universals.....................................................................................................109

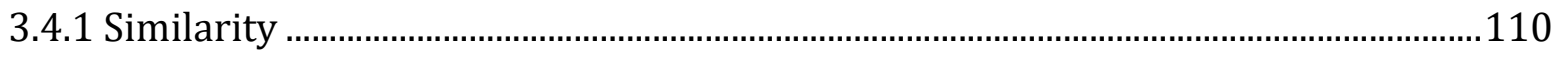

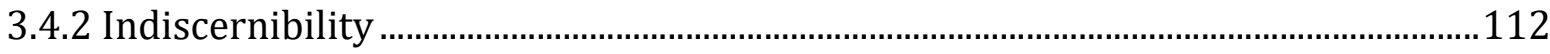

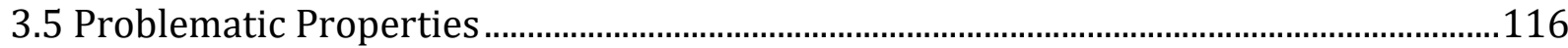

3.5.1 Accidental and Essential Tropes..............................................................................117

3.5.2 Spatiotemporal Properties ...........................................................................................118

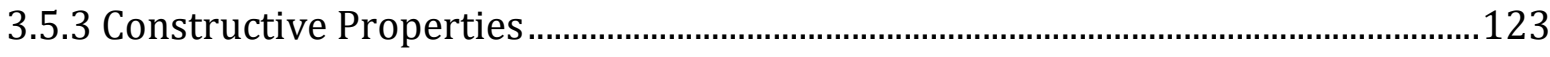

3.5.4 Foundational Tropes...............................................................................................125

Chapter 4: Scientific Laws and Constitutive Trope Theory …………………………………...131

4.1 Deterministic Laws ..........................................................................................................131

4.1.1 Dependent Laws .....................................................................................................132

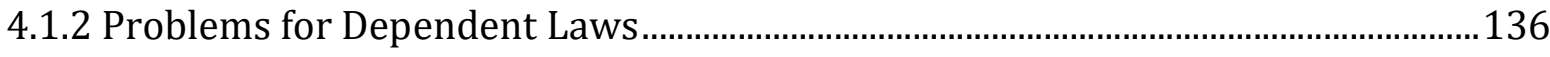

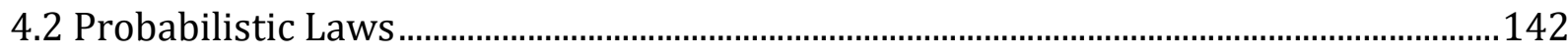

4.2.1 Irreducibly Probabilistic Laws ........................................................................................144

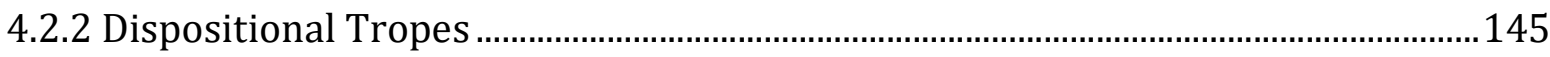

4.2.3 Indiscernibility and Dispositional Probabilistic Tropes ...........................................152

4.2.4 Reducibly Probabilistic Laws........................................................................................155 
4.3 Dispositional Essentialism.................................................................................................

4.3.1 Nomic Generalizations .............................................................................................

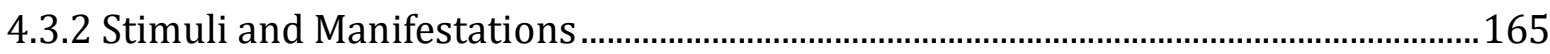

4.3.3 Finks and Antidotes..................................................................................................167

4.3.4 Ceteris Paribus Laws...................................................................................................170

4.3.5 Weak Realism About Laws .......................................................................................177

4.3.6 Problems for Dispositional Tropes..............................................................................180

Chapter 5: Problems and Final Considerations.........................................................................185

5.1 Problems for the Account......................................................................................................186

5.1.1 Foundational Tropes..............................................................................................187

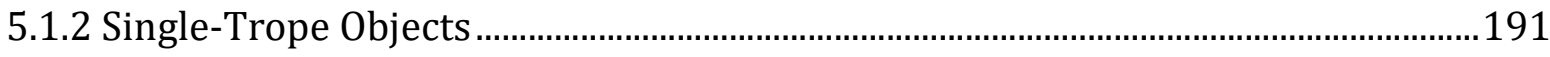

5.1.3 Dispositions vs. Universals ..........................................................................................194

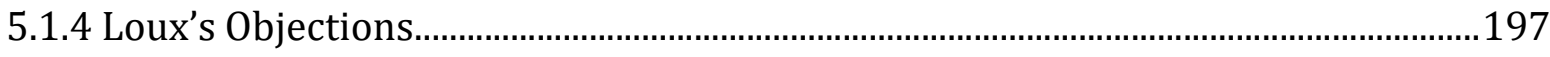

5.1.5 Justifying Commonality ..................................................................................................201

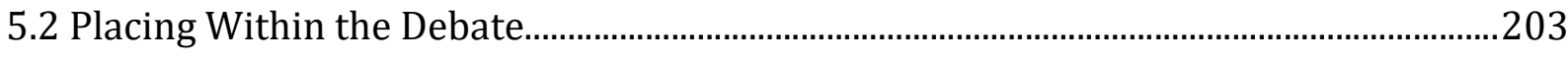

5.2.1 Number of Properties ...............................................................................................205

5.3 Falsification of Constitutive Trope Theory.......................................................................207

5.3.1 Falsifying the Constitutive .......................................................................................215

5.3.2 Falsifying via Foundations .....................................................................................218

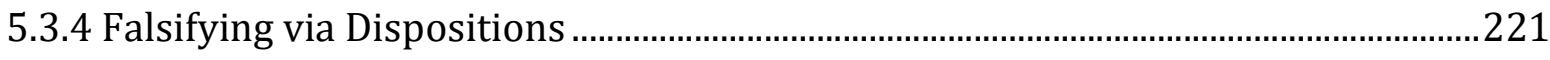

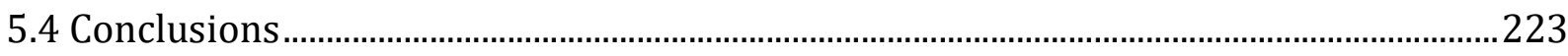

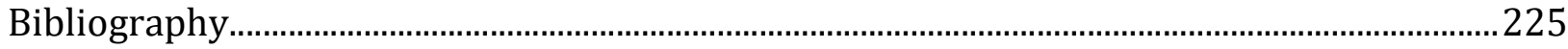




\section{Introduction}

The success of the sciences in describing the world is, if not undeniable to the philosopher, at least something that every philosopher must contend with. Some have gone so far as to claim that the need for philosophical analysis is unnecessary, to be replaced with scientific investigation, although how successful such projects are is not unironically a subject of intense philosophical debate. But even such projects must answer a few key philosophical questions that we might then rely on scientific evidence, whether we do so in addition to or in replacement of philosophical analysis. For metaphysics, one such question is the question of how the world is such that scientific theories can describe it accurately. It is this question that is the primary question of this work: what is it about the world that makes it capable of being described successfully by scientific theories.

Aside from satisfying one's curiosity, an answer to this question may further prove useful. If scientific theories are successful in describing the world then this may provide both a useful window for solving a number of problems related to the objects of scientific theories and provide a criterion for evaluating the success of such answers. And there is no shortage of such problems. Philosophical puzzles of object composition, on the nature of minds, on the nature of time and space, and other such deep puzzles are either about objects of scientific investigation or involve those objects heavily. Thus, answering the question of what it is about the world that makes it capable of being described by science is not only an intellectual puzzle but also a fruitful avenue for investigating a number of other problems. 
This question is often answered by answering the question of what grounds the truth of (successful) scientific theories. If there is something about the world such that scientific theories constitute accurate descriptions of the world, then the fact of their success is less mysterious, at least with respect to how they relate to the world. If one believes that describing the world at this ontological level is impossible on epistemic or methodological grounds, then other avenues may be investigated but even this constitutes an answer to this question if only in saying that there is nothing that does this, or that the truth of scientific theories are grounded in some other way.

This means that to answer the question of how it is that the world is such that scientific theories can accurately describe it requires some theorizing on the nature of the things that are in the world. And I am not alone in this. David Armstrong, C.B. Martin, and others who have attempted to answer this question provide answers that come attached to theories about the nature of the entities in the world. Examining these theories is useful for two reasons. First, if such a theory is successful than there is no need for further investigation. However, this may not be as easy as it sounds as deciding what makes one theory of metaphysics successful or not is itself a debate: one that I will discuss in Chapter 1. As it will turn out, there are many different dimensions of success to consider, and so a theory may prove to meet some of our criteria and not others. Deciding the best theory from amidst multiple divergent criteria is no easy task, but we might hope that one account proves far and away better than its competitors. The actual examination of these theories will take place in Chapters 2 of this work. Due to the number of theories that have been advanced by philosophers, a full accounting of all such theories is neither possible nor desirable in this space. This brings me to the second reason for examining extant theories. 
That even if no theories are successful or successful enough than examining those theories may prove useful in the creation of a successful theory. This second criterion is largely responsible for the choice in theories that I have examined here, as the more influential and developed theories will provide more material both for determining whether the theory is successful and for showing potential pitfalls to avoid in the creation of a new answer. As I do not find the current accounts in the literature satisfactory, rather than defend one of those accounts I will be advancing a theory of object properties and how they ground scientific laws. This will be the project of Chapters 3 and 4 of this work. Chapter 5 will address some problems that arise in Chapters 3 and 4 that were not addressed at those times, as well as bring in one advantage of the theory that I believe it has over its competitors: its falsifiable nature.

Before continuing, I would like to include a brief index of certain terms that will become useful in the coming chapters. These terms and their associated definitions have been chosen to reflect the terms used in current debates regarding a metaphysics of properties and have largely been taken from Armstrong's usage, which has become standard among many philosophers studying this issue. Where possible, I also explain these terms in my work. However, by including them here in a sort of index I hope to make it easier for those referencing my work to find terms that may be confusing or technical.

Property- An aspect or characteristic of an object, such as its color, height, charge, etc. Property is meant in the most philosophically neutral sense possible and is not meant to imply the objects of a particular theory or even the existence of a metaphysically real entity. 
Object- Like property, object is meant in the most philosophically neutral sense possible. While providing an exact definition of an object is difficult without referencing a particular theory of properties, objects can broadly be described as those things which bear properties. Physical objects such as tables, beds, and philosophy papers are common examples of objects, as are entities such as electrons and quarks, which may not be detectable without some special apparatus or tool.

Universal- A property that is or can be present across distinct objects and/or spatiotemporal locations while being the same property. Theories which accept the existence of universals are called realist theories, as this is the term used in the literature.

Trope- A property that can only be present in a single particular instance. Unlike a universal, a trope cannot be present in different objects or locations. Theories which accept the existence of tropes but not universals are called trope theories.

Nominalism- Nominalist theories are those which accept neither the existence of universals nor tropes, though they may accept the existence of objects. Nominalist theories will as a rule try to explain the appearance of properties but will not accept metaphysically real properties. 


\section{Chapter 1: Motivation, Method, and Desiderata}

On the question of properties, there are two major questions that one may ask. One is the question, "What sort of properties are there?" and the other is the question of "What does it mean for something to be (or have) a property?" This second question may seem to be more of a linguistic issue than an ontological one: a matter of the meaning of a word rather than a matter of metaphysics. One might wish to replace it with a more obvious metaphysical question of "Are there properties?" But there is no one theory of what properties are and attempting to work from a mere linguistic definition runs the risk of missing the point. If one defines properties as multi-present entities, for instance, then even if one shows that there are no such multi-present entities one has failed to show that there is nothing in the world that corresponds to our pre-theoretic notion of a property. As such, before we can answer the question of whether or not properties exist, we must find an answer to the question of what it means for there to be a property. Thus, despite using the word 'mean' in the above question, this is not primarily a linguistic question but an ontological one. The question is about what sort of thing a property is, and if there are no such things in the world as properties, how it is that objects appear to have properties.

The former can be asked in either a scientific or a philosophical sense. The (perhaps more common) empirical response to the question is an enumeration of qualities that an object may be supposed to possess: height, length, charge, color, emotional charge, etc. However, there is also a philosophical response to the question, as it may turn out that some properties are not legitimate. For instance, many philosophers have supposed that 
something having a color or being emotionally charged is not a genuine sort of property. There is a reflective equilibrium between the empirical and philosophical senses: if a property is found to be explanatory in empirical investigation, philosophers then are more inclined to count it among the 'genuine' properties or their critics will at least demand of them to explain the explanatory power of the property given that they think it is nongenuine.

To further separate out these questions and make them transparent, we can look at a particular instance. We will start with a philosophically naive or commonsense view of properties: while this may not end up being what philosophers are after, it provides us with a starting point with minimal theoretical commitments. Take my desk: it is 30" tall, brown in color, and its surface has a rectangular shape. This list, along with a list of other properties the desk has, forms an answer to the empirical question, "What are the properties of the desk?" The question, "What does it mean for the desk to have a property?" will then ask what it means for a desk to possess properties at all. And in this investigation, it may turn out that some properties that are discovered empirically, such as the desk's color, are ontologically suspect. The desk may appear to have a different color under different lighting, or to a color-blind person, or in other circumstances that make us question whether the desk truly has one property that is its 'color' or if the observed color should be explained in some other way. Locke draws the distinction between primary and secondary qualities in this way, where primary qualities are those properties an object has in and of itself, and secondary qualities are those properties an object appears to have in 
virtue of its primary qualities and the object's relationship to other objects. ${ }^{1}$. Using this distinction, we may ask whether certain properties of the desk are primary or secondary qualities. As the primary qualities of the object provide the ontological ground of the secondary qualities, we may use this distinction to answer the question of what sorts of things properties are and how objects possess them gives us philosophical grounds to eliminate or replace certain empirical properties with ones that are less-suspect. This process is also often informed by empirical investigation: for instance, we might think that the light-reflecting properties of the molecules of the desk ought to be examined as primary qualities in lieu of what we colloquially think of as the 'color' of the desk for scientific as well as philosophical reasons.

It is this latter question of what it means for an object to have a property that is the primary subject of investigation here. However, as seen in the above example, this will inform the earlier question of what sorts of properties there actually are. There will be other theoretical consequence of the acceptance of the theory being developed, and some of these consequences will be discussed in more depth. For now, it is enough to have an idea of the question we are investigating.

\subsection{The Problem of Universals}

One theoretical consequence of a theory of properties that philosophers seek out is an answer to a question known as 'the problem of universals.' While it has been stated in many different forms, the problem of universals is a question about the similarity we see in

\footnotetext{
${ }^{1}$ John Locke, An Essay Concerning Human Understanding, ed. Kenneth P. Winkler, (Indianapolis, Hackett, 1996)
} 
the world around us. Following Armstrong and Peirce, philosophers generally examine this in terms of types and tokens. Take a case where we have two apples in an otherwise empty box. We might ask how many things are inside that box. Armstrong points out that there are two answers: ${ }^{2}$ there are two different objects in that box, but there is only one type of thing in the box. This sort of apparent similarity in the world around us is what an answer to the problem of universals attempts to give us. If we turn our attention to properties, we may examine a case of two apples that are the same shade of red or two particles with the same charge. Each has a different token-property of color or charge, but the same type of property. This problem also arises for properties which do not perfectly resemble one another. For instance, take the property of being 6 ' tall and the property of being $7^{\prime}$ tall. These are certainly not 'the same' property in the sense above, but each is more similar to the other than to the property of weighing $200 \mathrm{lbs}$. or the property of being purple. In terms of types and tokens, each shares the type of being a height, which it does not share with the mass or color properties later mentioned. Using the type-token distinction, the problem may be put in these terms: in virtue of what do two objects belong to the same type?

This question is striking for two reasons. The first is that in practice we categorize the world in terms of similarity. That two objects are identical with respect to some type is an underlying fact that is used almost without consideration in everything from scientific theories to everyday interactions. And theories of properties such as Armstrong's have been used to at least attempt an answer to this question. As such, any competing theory that does not provide some answer to the similarity problem is going to lack explanatory power in comparison to a theory like Armstrong's. This provides some impetus for anyone

\footnotetext{
2 David Armstrong, Universals: An Opinionated Introduction, (Boulder, Westview Press, 2010)
} 
concerned with questions about properties to at least attempt an answer to the problem of universals. As such, this will be one desideratum for such investigations that we will use in evaluating such theories.

\subsection{Scientific Laws}

While not all philosophers who analyze what it means to have a property are concerned with scientific laws, many have taken it as advantageous that their account of properties can give us an account of scientific laws. Armstrong's theory of properties as universals, for instance, attempts to use his theory of universals to analyze what the ontological basis is of scientific laws. Like our earlier question about the nature of properties, there is a corresponding question regarding scientific laws; namely, what is it for something to be a scientific law? And like our earlier question on properties, there are many ways this question might be answered. A linguistic answer, for instance, will say something to the effect that scientific laws are statements about reality made by scientists that are purported to be accurate descriptions of phenomena in the world. While perhaps true, this sort of answer is of little interest to an ontological project. To rephrase the question in an ontologically interesting way, we may ask, "What feature of the world makes scientific law-statements true?" If one doesn't like 'true' here, they may substitute for 'accurate' or even 'useful.' It would be strange, and perhaps a mark against ontology as a discipline, if in studying what it is for the world to be as it is that we cannot provide any reason scientific law-statements are so successful in describing the world around us.

One might think that this is a false worry, and some philosophers have questioned the real place of laws in science. Russell himself said that many laws of nature are either 
mere human conventions or simply statistical averages that do not require special ontological explanation. ${ }^{3}$ Nancy Cartwright has pushed for an even stronger criticism. Cartwright is critical of what she terms 'theoretical laws' in sciences: laws that cover phenomenon that are not directly observable. Cartwright believes that scientific lawstatements, where they are not mere descriptions of observable phenomenon, ought to be treated as models of reality and says that no one model can capture all aspects of a phenomenon and thus, no one model- that is, no one set of laws- is better than any other. ${ }^{4}$ This is not to say that there are not certain models that are better at describing a phenomenon than others, but rather that there is no single, universal set of laws that will consistently be best for all phenomena or even every aspect of a particular phenomenon. On this view, there is no reason to grant any special ontological status to scientific laws. For Cartwright, these laws are simply postulates that have proven effective at capturing some aspect of a phenomenon but there is no special reason to think these models are true of the world: there are other, contrary models that may serve to describe a phenomenon just as well..$^{5}$

A related worry may come about with respect to sciences other than physics. ${ }^{6}$ If one is attempting to give an ontological grounding for all of the sciences and not merely physics, laws may not be of great importance. For while physics, and perhaps chemistry, deal largely in laws, in many other sciences they hold little significance whatsoever. So, an ontological

\footnotetext{
${ }^{3}$ Bertrand Russell, Why I am not a Christian and Other Essays on Religion and Related Topics, (London, George Allen \& Unwin Ltd, 1957).

${ }^{4}$ Nancy Cartwright, How the Laws of Physics Lie, (Oxford, Oxford University Press, 1983)

${ }^{5}$ Cartwright notes that these might be the same aspects of the phenomenon in question: one model might be effective at capturing some aspects of a phenomenon while another might capture some other aspect, leading to competing theories that are nonetheless accurate.

${ }^{6}$ I am indebted to Carrie Figdor for making this objection explicit.
} 
analysis of laws may not be the answer to providing an ontological ground is for the success of scientific practice. One might respond, and it seems many philosophers studying laws have implicitly responded by either ignoring 'higher level' sciences in favor of studying chemistry and physics, or by arguing that regularities and success in the higher-level sciences reduces to interactions in chemistry and physics which are accurately reported in law-statements.

To address Russell's arguments: in the case of 'human conventions' such as a yard being equal to 3 feet, I agree ${ }^{7}$. Any such conventions need no specific ontological grounding, as they are merely analytic of arbitrary conventions. However, in the case of reducing laws to probabilities I must argue that there needs to be a lawlike relation between the probability in question and the properties to which the probability applies. Take the case of rolling an unweighted die with 6 sides. It would be unsurprising to find that it lands on a particular face 1 in 6 times, on average. But this is only the case once we know something of its weight distribution and the number of sides it has and how these influence the probability of it landing on a particular face. If the die has 8 sides, or was weighted very heavily in a particular direction, these facts would alter the probability. So, one cannot get lawlike probability relations without some reference to the properties of the object in question, meaning that one still needs an ontological basis for those probabilities.

In response to Cartwright's arguments, I agree that scientific law-statements are explanatory models of the world. What I find suspect is the idea that there is no one model that could capture all aspects of a phenomenon. Cartwright points to difficulties of

\footnotetext{
${ }^{7}$ We might wonder if this ought to be considered a scientific law-statement at all, given that it is merely a convention of measuring. Russell refers to this case in dismissing certain laws of nature, and so I am including it for completeness.
} 
capturing all aspects of phenomena described by theoretical physics. For instance, relativity theory and quantum mechanics have proven notoriously difficult to bridge. But the mere fact that such a bridge currently evades us is not evidence that they are incommensurable. Cartwright could respond that it is the best that we can do: there is a common argument that "one cannot prove a negative." That in the absence of such bridge-principles being known, that the burden of proof lies entirely on one who wishes to posit them. But such a proof that there is no one, overarching model that describes physical phenomenon accurately is not too high a bar: we have cases where we can show such limitations in other fields, such as Gödel's proof that mathematics cannot be derived solely from logic. And success in some fields, such as quantum field theory, may give us reason to think that such an overarching model may be forthcoming. Further, for some phenomena the relevant scientific laws are well-known. The law of definite proportions in chemistry has no known counter-example and no model of chemical composition would be accurate without it or something like it: any competing theory which says that how a chemical compound is made (aside from the elements that make it) has an effect on the nature of the compound or which was quiet on this subject would not be as accurate as one that includes the law. That at the frontiers of scientific inquiry we find difficult questions to answer only tells us that scientific inquiry is not instantly resolved. It tells us nothing about what answers or lack thereof we may find.

Finally, in response to the above line of reasoning: it is true that laws find their use more in the physical and chemical sciences than in biology, psychology, economics, or other 'higher level' sciences. However, this does not mean that there are not laws in these higherorder sciences. While there is philosophical work that needs to be done before one can 
commit to which statements of science are laws, there are many such statements in these higher-order sciences that qualify. Biology features Rubner's Law, Bergmann's rule, Kleiber's law, and dozens of others. Psychology features Weber's law, Stevens' power law, the Matching law, and other principles that may well fit. Economics features the Supply Law, the Law of Diminishing Returns, and other candidates for ontologically significant laws. And it is the same way in other sciences. There is debate about whether or not these ought to be counted as laws in the same sense as laws of physics or chemistry. An ontological grounding of laws serves two purposes with respect to such a debate. First, if the laws in question cannot fit into such a framework, they may be suspect. Second, if the laws in question do not fit into such a framework, we may delineate between sciences that study laws and those that do not, at the very least. Additionally, if an ontological framework cannot give us an acceptable reason that we ought to reject these as laws, such a framework may be found suspect. But given the sheer number of candidates for laws in the 'higher level' sciences, it seems that the objection that these sciences do not deal in laws has few teeth.

\subsection{Simplicity}

We have now examined three things that we hope an account of properties will be able to accomplish: to answer what it is for an object to have a property, to give an answer to the problem of universals, and to give an ontological grounding for scientific laws. But not all accounts that set out to perform these tasks will be equal. This is not surprising: in every discipline, philosophers do not merely advance new accounts but debate the relative merits of accounts that have been advanced. There are three concerns that I believe are 
most central to evaluating an account here, and an examination of these is in order before we continue. These issues involve simplicity, primitives, and what I will term "philosophical baggage."

One concern advanced in the literature is that of ontological simplicity. But this is not a simple matter. Yet it is a concern among philosophers and we will thus discuss it. Armstrong gives it as the primary critique that the nominalist advances against the realist. ${ }^{8}$ This is usually advanced in an Occamist manner, with the rule being stated as "all things being equal, one should not multiply entities beyond necessity." To put it another way: one should not postulate more entities than one needs to explain a given phenomenon. While giving a full defense of an account of simplicity is beyond the scope of this work, there are at least two primary issues here that need to be addressed: what counts as being equal and what constitutes necessity for a theory of properties, and when we talk about multiplying entities are we talking about types or tokens?

The first issue comes down to explanation. The reason we give an account of properties is that we hope that it will give an accurate explanation that will adequately answer certain problems. In the course of this, a philosopher may postulate the existence of certain kinds of entities to do that work: classes, universals, relations, or others. If successful, this explanation will adequately answer the problems that the philosopher sought out to give an account of. The philosopher must postulate some entities or kinds of entities to give an explanation, they have excuse to do so provided that those entities are necessary for the explanation given. Given that this is required for an account, one can think of conditions such as "all things being equal" as granting some leeway to the

\footnotetext{
${ }^{8}$ Armstrong, Universals: An Opinionated Introduction.
} 
philosopher. But not all theories will be equal. Take a theory that requires there to be four distinct kinds of entities: say, transcendental universals, particulars, relations, and minds. Further, let's say that on this account none of these are a "kind" of the other: relations and minds are not to be understood as a kind of particular but as fundamental to the theory in question. Now consider an opposing theory that performs all of the same explanatory work, but only postulates the existence of transcendental universals and particulars. Relations and minds, where they factor into the theory, are analyzed as kinds of particulars or transcendental universals. If the two theories perform the same explanatory work, there appears to be no reason to prefer the former to the latter, and a good deal of reason to prefer the latter to the former. For one, any philosophical problems attached to accepting the additional entities as primitives of the theory will attach themselves to the former theory but not the latter. But additionally, there seems to be no reason that we need accept the additional entities as we have an equally explanatory theory that does not utilize them. While the idea that a simplicity criterion ought to be applied to philosophical debate is not particularly controversial, which simplicity criteria are relevant to philosophical discourse is a difficult matter. The criterion defended is a traditionally Ockhamist one, and is taken by Huemer, ${ }^{9}$ Lewis $^{10}$, and Nolan ${ }^{11}$ to be a quintessential criterion for simplicity. Even Lewis and Nolan, who wish to modify or alter the principle in some way, do not question the principle itself, instead making the more modest claim that there are other relevant kinds of similarity (quantitative simplicity for Nolan) or modifications to the basic principle (for

\footnotetext{
9 Michael Huemer, “When is Parsimony a Virtue?” Philosophical Quarterly 59, No. 203, (Apr 2009): 217.

${ }^{10}$ David Lewis, Counterfactuals, (Blackwell, Oxford, 1986): 76.

${ }^{11}$ David Nolan, "Quantitative Parsimony." The British Journal for the Philosophy of Science 48, No. 3 (Sept 1997): 330-1.
} 
Lewis) that need to be considered. Aside from those who wish to modify or add to the principle, there are competing notions of simplicity that one might defend, such as Sober's 'minimum available information' criterion of theoretical simplicity. ${ }^{12}$ Defending the Occamist notion of simplicity would be a work unto itself, and so I will do something more modest and attempt to show why this version of simplicity is particularly relevant to metaphysics of properties.

Unlike other debates where simplicity is relevant in philosophy or the sciences, in metaphysics of properties we are focused on one question: what it is for an object to have a property? This constrains the notions of simplicity that are relevant. Consider Nolan's quantitative simplicity ${ }^{13}$, which states that in addition to assuming only the minimal number of theoretical entities, we should favor theories that assume only the minimum number of entities full-stop. ${ }^{14}$ But for the question of what it is for an object to have a property, this distinction is less relevant. A theory of properties ought to be valid in a world whether the world contains one entity, or two billion, or two googleplex. So even granting Nolan's criterion to be valid generally, it is not relevant to the current question at hand. Similarly, when postulating particular kinds of properties in the world, a criterion such as Sober's 'inductivist formulation' 15 may well be relevant to deciding between two theories regarding those entities. But while this may be relevant for theory choice between theories which utilize properties, it is not relevant for theory choice between theories which

\footnotetext{
12 Ibid: $330-1$.

13 Ibid: 340-1.

${ }^{14}$ This distinction will be explored later in this section.

${ }^{15}$ Eliot Sober, "The Principle of Parsimony." The British Journal for the Philosophy of Science 32, No. 2 (June 1981): 153.
} 
describe what it is to be a property, as the discussion is itself about the kinds of entities that exist, rather than about detailing the nature of those specific entities.

And this is why the Occamist criterion is relevant here. The question of 'what properties are' is specifically about what kinds of properties we need to postulate for a theory. As such, assuming only the minimal number of theoretical entities is particularly relevant for the project. If two theories can do the same explanatory work, with one needing to postulate 5 kinds of entities to do the work that another theory can do with just one, the latter is preferable as we need only commit ourselves to one kind of thing. As our current question is specifically about what kinds of entities we ought to be committed to, this particular criterion of parsimony is well-suited to the question at hand, far more so than its rivals. As such, even if we acknowledge the usefulness and even primacy of other kinds of parsimony for other investigations, this Ockhamist notion of parsimony is most relevant here.

To spell out this notion of parsimony more distinctly, I will distinguish here between two kinds of entities: observational entities and theoretical entities. Observational entities are just the entities observable by the unaided senses. Their justification is entirely a posteriori: we know that these objects exist through observation alone. One might bring up classic Cartesian worries here: the objects that we know could be the products of an evil demon, a hallucination, or otherwise be false. But even in the Cartesian case, the existence of the thing as an image is not itself an illusion: even if a tree is only an appearing, there is still an appearing, even if not a 'tree.' There is still the appearance of an object. We may have different theories about why we have this appearing instead of that one, but the appearance of an object is still present. However, observational entities grant us a 
completely unexamined view of the world: we cannot explain how a tree grows by mere observation, or even that a tree grows, or even that it belongs to a kind 'tree.' At best, we can identify that at a certain space and time there was an object of one size and at another space and time there was a similar object of a different size, but even this explanation is rife with theoretical terms: similar, object, different, space, time, etc. This I take to be a facet of our language: language exists in part to impart ideas, and implanting ideas requires some amount of explanation. But before language, there is an object of the observation. ${ }^{16}$

But we cannot explain the world that we see by positing only those objects that are picked out by direct observation. Understanding how a tree manages to grow requires knowledge of photons, cells, chlorophyll, and photosynthetic processes, among other things. When I look at a tree, I do not observe any of this: I might be forgiven in my observation, if not my education, for not recognizing any of this when I look at a tree as it grows. So, we posit other entities that explain the behavior of the observed entities or posit some facet of the entity we observe that remains hidden from us. These are theoretical entities.

In the sciences, when a theoretical entity is posited, there is an expectation that some future experiment may show that the entity exists or does not exist: that the assumption of the entity carries with it observational consequences that we may test. For entities assumed in philosophy, in particular in metaphysics, we are not usually so fortunate. It is not clear what, if any, are the consequences of assuming that there are

\footnotetext{
16 There is an interesting question here about which observations are direct and how much of our observation is theory laden. This is important philosophical work, but outside the scope of this project. All I require for this distinction is that there are some entities which we know through observation and others which we know only or primarily through their role in explanation.
} 
uninstantiated universals, or tropes, or qualia, or a number of other entities as opposed to theories which do not assume them. However, we can rule against assuming unnecessarily that such entities exist. If we can do all of the philosophical work we need to do by assuming one theoretical entity rather than two, then there is no reason to assume the existence of the extra entity.

There is a further complication, here as there are still many questions to be asked about simplicity. Is it better to assume many distinct theoretical entities of a kind, or fewer theoretical entities of many distinct kinds? What of kinds of entities that are distinct, but fall under some more general category? What should we sacrifice when simplicity conflicts with the other desiderata of this account? These are tough questions, and there will be many penumbral cases that we can imagine where answers to these questions may be necessary. However, the following general rules can be given as conforming to what are generally taken to be guidelines of simplicity, both in and out of philosophy. First, positing one kind of entity with many sub-kinds is less egregious than positing many distinct kinds of entities. We can see the examples of this in classification schemes in biology, chemistry, and physics, where the best explanations recognize general kinds such as 'atoms' and then sub-kinds such as 'hydrogen' and 'oxygen' rather than positing each particle as its own unique kind. Second, that in general, positing many theoretical entities of a kind is less egregious than positing multiple kinds of theoretical entities. This rule is less hard-and-fast than other rules, particularly in cases where we are asked to add an infinite number of entities as opposed to admitting a new kind. ${ }^{17}$ These cases are harder to adjudicate, but I

\footnotetext{
${ }^{17}$ I have in mind here views such as Lewis' 'possible worlds' view or certain mereological views of the nature of matter.
} 
think there is enough commonality between thinkers to allow each case ought to be argued individually on its merits and where no one systematized set of rules will adequately capture simplicity in its entirety. We can hope that some account will prove to be far and away better than others in this regard, especially when combined with other desiderata, but there is no guarantee that we will have as easy a time as that.

\subsection{Philosophical Baggage}

Other issues involve those of philosophical primitives and what I will call philosophical baggage. As I take these issues to be tightly linked, I will be examining them together. But first we must understand what it means to be a primitive. This comes back to understanding explanation. A theory will attempt to provide an explanation or account of some phenomenon. To give this sort of account, certain terms will need to be used. These terms may themselves admit of an account or explanation, but then the terms used in those secondary explanations will also need an account. There seem to be only three ways this can end for an account. The first is for there to be an infinite descent of terms, each explaining the other. The chief problem with this sort of explanation is that it is not clear that it can do the job of explaining. If $A$ is understood in terms of $B$, and $B$ in terms of $C$, and so on ad infinitum, then each term is reliant on some other term and no term is adequately explained. The second is to eventually (perhaps immediately) provide an explanation using some previous established term. To give an example, one might explain objects in terms of properties, and then explain properties in terms of objects. But this sort of explanation is generally regarded as unsatisfactory. If my explanation of objects is entirely in terms of properties, and my explanation of properties is entirely in terms of objects, then it seems 
neither is actually explained. The final strategy is to use some set of primitives: entities which the theory does not attempt to give an analysis of but uses to explain other entities described in the theory.

To be clear, I believe that in order to avoid circularity or an infinite descent in explanation an account must have some primitives. As such, philosophers are free to choose whatever primitives they like for their account. But I believe it is important to note that merely calling something a primitive does not explain that thing. To use a relevant example, if I purport to answer the problem of universals, which is a question of how two token properties or objects can be similar with respect to type, if I declare similarity to be a primitive of my theory then I have not succeeded in giving an analysis of similarity. I might still be able to perform some work with the theory by showing how, once similarity is accepted as a primitive, one can account for similarities between properties or objects. And this might still be an adequate solution to the problem of universals. But I cannot then claim to have explained what it is that makes two things similar. In fact, a philosopher who does this must then accept that they have no such explanation, at least not in the account given. And this will make their account weaker than one that can provide such an explanation.

But different philosophers use different notions of primitives, and I believe there is an important distinction to be made between two kinds of primitives. The first I will refer to as unanalyzed primitives, the second as unanalyzable primitives. The distinction here can be a subtle one and it is not always clear which of these a philosopher is using. An unanalyzed primitive is an entity that the theory does not explain but uses in its explanations. But when a philosopher postulates an unanalyzable primitive, he goes further than this and says that there can be no explanation for the entity in question: the entity can 
admit of no explanation. Putting my cards on the table, while I accept that unanalyzed primitives will be necessary to avoid an infinite descent or a circular account, I am highly suspicious of unanalyzable primitives and do not believe that they succeed in explaining the phenomena they purport to explain.

My argument for this is as follows. Imagine that you have a theory that explains in terms of an unanalyzable primitive $\mathrm{X}$. As $\mathrm{X}$ is unanalyzable, we cannot say anything about its internal nature: any such statement would be to give an essence to $\mathrm{X}$ which would allow for some analysis. But this then infects all explanations regarding X. As X cannot be analyzed, those things which are explained in terms of X have the mere appearance of explanation. If I explain causal phenomena in terms of widgets but then tell you that not only will I not provide an explanation for what widgets are, but that widgets are inherently unanalyzable, then it's not clear how widgets can do any explanatory work at all. At least if I make the more modest claim and say that I have not provided an analysis of widget-hood but allow for the possibility of such an explanation then I have opened the door for a meaningful analysis in terms of widgets.

I think this critique of unanalyzable primitives is clear enough when talking about widgets or a variable such as X. I think where philosophers lean in to unanalyzable primitives is when they give their primitive a name that is meant to evoke intuitions about the nature of the thing despite us having been told that it is unanalyzable. If I don't call the unanalyzed primitive 'widget' but instead refer to it as a class, a concept, a universal, or by some other word then I invite the reader to use their intuition to fill in the nature of the thing while on-paper being committed to it not only lacking definition in my work, but in it in-principle being indefinable. In addition, as the unanalyzable primitive admits of no 
further analysis it allows one to "bottom out" their ontology (or other philosophical project) in a primitive and thus avoid charges that the account may lead to an infinite descent or circularity.

But aside from the earlier issue of primitives and their lack of internal explanatory value, I do believe that philosophers may choose whatever unanalyzed primitives suit their project. However, the more unanalyzed primitives one has, the more that will need to be explained in future accounts. And this falls into a general category of what I like to call 'philosophical baggage.' Generally speaking, I use philosophical baggage to refer to a variety of problems that a theory either does not solve or which contradict either our intuitions or accepted theories about the world. Unanalyzed primitives fall into the former, as they provide work that future supporters of the theory (if there are any) will need to perform in order to provide a more detailed account. The latter, however, are also common in philosophy. There are a variety of concepts in ontology which carry this sort of baggage: possible worlds, atomless gunk, essences, and others have all, at various points, been challenged on account of the philosophical baggage that accompanies them and theories that utilize these concepts often find criticism from those who don't believe that baggage ought to be brought along. Similarly, if a theory implies that objects do not exist, that time is an illusion, or some other theory that drastically contradicts our perception of the world then many will criticize a theory for failing to adhere to our intuitive understanding of the world around us.

Now, the mere fact that such criticisms exist is not itself decisive. Many philosophers simply choose to bite the bullet and accept that their theory has some unintuitive consequence or admits of a world radically different than what we thought. And most 
theories will carry at least some philosophical baggage in the form of unanalyzed primitives if nothing else. So, criticisms regarding the philosophical baggage that accompanies a theory are more about which things we are willing to accept as work for a theory and consequences of accepting a theory and which things we are not. Unfortunately, there is no clear way to weigh the baggage of a theory under consideration. If we are lucky and have two nearly identical theories save that one has an extra unanalyzed primitive, then we might prefer the theory that does not carry that baggage. But if one theory requires that we accept atomless gunk and another requires we accept monism and yet another requires that we accept possible worlds then it is not immediately clear which theory would win out given these criticisms. All one can do is attempt to show that their theory does not have the problems of other theories and do their best to minimize the baggage that the theory they defend brings with it.

\subsection{Desiderata of an Account}

The above sections lead to a defense of five desiderata for an account:

1) Answer what it is for an object to have a property;

2) Answer how it is that two different objects can have similar properties, that is, the problem of universals;

3) Give an ontological basis for scientific laws;

4) Assume only the minimal number of theoretical entities needed to provide an account; and

5) Offer a comparative less philosophically problematic theory compared to rival accounts of 1-3. 
I would like to call special attention to the third desideratum at this point, as it will be relevant in my choice of which theories I will be examining. Certain theories of properties do not give an ontological basis for scientific laws. In some cases, this is because they are solely concerned with the first two desiderata and do not see the third as a part of their work. These theories might admit of an ontological basis for scientific laws, if someone were to take on that extra work. I will not be examining these as it would be unfair to attempt to weave whole-cloth what another philosopher might be committed to if they were to provide an answer to the third desideratum. Thus, while such theories may certainly be worthy of consideration they will not be examined in this work.

There is another reason theory of properties would fail to provide an answer to the third desideratum. This is that they do not take there to be an ontological ground for scientific law statements and so do not think it is the work of a theory of properties to do so. I have discussed such views briefly in my defense of the third desideratum above. But given my commitment to the third desideratum, I will not be examining views that do not attempt in some manner to give an ontological basis for scientific laws. As most of the views which take this approach are nominalist in nature, this means that realists and trope theorists will be given more consideration than nominalists. I find this side effect to be acceptable and hope it will be for my reader as well. 


\section{Chapter 2: Theories of Properties}

There are already numerous theories of properties, so to begin with we must investigate which of these, if any, are adequate for our needs. While it would be impossible to do a full recounting of every theory of properties here, I will attempt to show in the broad strokes some of these accounts and their advantages and problems. To begin with, we ought to describe what is meant by a property. Philosophers vary on how they identify properties with specific distinctions often related to the project pursued. For instance, Armstrong makes use of the type:token distinction with properties being grounded in the "relativity of identity of types,"18 while Campbell identifies them as "abstract particulars," although his use of the term 'abstract' here is idiosyncratic. ${ }^{19}$ Here I will attempt to be as philosophically neutral as possible and say that properties are naively identified as characteristics of an object capable of overlapping one another in a spatiotemporal area. For instance, a single object may have a mass, area, color, texture, etc. I do not intend for this to do any philosophical heavy lifting. Properties so defined may be universals, tropes, transcendent, imminent, or even non-existent. My use of the term properties is also not meant to convey anything we must be committed to in our ontology. Rather, it is meant to capture our pre-philosophical notions of what it means to be an attribute of an object.

\footnotetext{
${ }^{18}$ Armstrong, Universals: An Opinionated Introduction.

${ }^{19}$ Keith Campbell, Properties, (Oxford, Blackwell, 1990).
} 
While there is some disagreement, most notably with trope theory, philosophers doing work in this field generally recognize three kinds of theories of properties. These are:

- Realism: The view that properties exist, and that different objects can share a property. That is, that a single property can be multiply instantiated. These multiply instantiable properties are termed universals.

- Trope Theory: The view that properties exist but are never multiply instantiated. These singularly instantiated properties are termed tropes.

- Nominalism: The view that there are no properties. The fact that objects appear to possess them is explained without positing actual properties.

The term 'realism' is then here used for a realism about universals, rather than a realism about properties. Again, here I am only trying to utilize the terminology of others working in this same field. It is notable that a theory of properties cannot belong to more than one of these classifications. On this taxonomy, any theory that posits the existence of multiplypresent properties is a form of realism, regardless of whether there are few or many such multiply-present entities. By contrast, any theory which denies the existence of properties is a form of nominalism. While most philosophers today use this taxonomy, trope theory is a sticking point on this. Many philosophers categorize trope theories as a sort of realism or nominalism as they either make the divide between theories that posit any sort of properties at all, or those that posit universals or not. ${ }^{20}$

\footnotetext{
${ }^{20}$ This means that on some views, trope theorists are nominalists while in others, trope theorists are realists. I find making trope theories their own category to be more useful as it removes the ambiguity.
} 


\section{1 Realist Theories}

We can now examine theories of properties to look for candidates which meet our desiderata. For realism, I will use the view put forward by David Armstrong as a starting point. While his is certainly not the only realist theory available, Armstrong's has the distinction of being one of the most referenced and one of the more fleshed out. Further, Armstrong makes clear attempts to answer the questions of the desiderata given in Chapter 1: Armstrong says what it means for an object to have a property, explains similarity, and explains scientific laws under his account. Thus, his account provides a good baseline for evaluation and we may use it as a springboard to examine other realist theories.

\subsubsection{Armstrong's Theory of Universals}

Armstrong recognizes two classes of entity ${ }^{21}$ : states of affairs and universals. A state of affairs for Armstrong is something like " $a$ is F" or " $b$ is $\mathrm{R}$ to $c$." The former is a description of the having of a property by a particular and the latter is of a relation between particulars, but for Armstrong both are states of affairs. To focus on the former case for a moment, Armstrong holds that there are universals: multiply-present entities which objects can share. This solves the first part of the problem of universals when we consider properties that vary only by spatiotemporal location. That any two electrons have matching charges is because they literally have the same property of charge, with that property being instantiated in two different locations. The 'is' in " $a$ is F" is what Armstrong calls 'the is of

\footnotetext{
21 'Entity' is a term used by Armstrong, intended to indicate something which exists but otherwise be philosophically neutral with respect to the kind of thing in question.
} 
instantiation.' To say that " $a$ is F" is not to say that $a$ is identical to $\mathrm{F}$, but rather that $a$ is a particular that has F.

But this appears to introduce a new kind of entity: a particular. That is to say, whatever it is that bears $\mathrm{F}$ in the state of affairs. We will consider potential problems with this story later, but Armstrong wants to draw a distinction between two kinds of particular here: a thin particular and a thick particular. The thin particular, he says, is some propertyless bearer of properties. This is what we normally think of as the $a$ in " $a$ is F." A thick particular, by contrast, is $a$ with all of $a$ 's non-relational properties. Armstrong says that this thick particular is itself the state of affairs, and it is this that he is committed to existing as an entity. He is less-clear on the existence of thin particulars as distinct entities. At times, he seems to want to dismiss them in favor of only talking about thick particulars. At other times, he says thin particulars are one of the constituents of a state of affairs. Again, we will look at this in more depth when we consider criticisms of Armstrong later in this chapter. For now, I believe it is best to leave him committed only to universals and states of affairs, or 'thick' particulars.

Having discussed the $a$ in " $a$ is F," we can now say more about the 'is': instantiation. Instantiation is vital to Armstrong's story: Armstrong does not believe that uninstantiated or 'platonic' universals exist. In other words, on Armstrong's theory, the universal exists only if there exists some state of affairs that instantiates it. Armstrong does not give an analysis of what it is to be a state of affairs, taking it to be a primitive of his theory. Armstrong uses states of affairs to answer where the universal is located. For Armstrong, the universal is wholly present in the states of affairs that instantiate it. 
This may lead to a problem for Armstrong. Critics have noted ${ }^{22}$ that universals existing only in the states of affairs which instantiate them could lead to universals 'popping in and out of existence,' as there may be times when a universal has no present states of affairs which instantiate it. Armstrong defends his view against this criticism by saying that as long as a state of affairs instantiating a universal has existed, there is no real problem here. ${ }^{23}$ The defense is that as long as some state of affairs instantiating that universal has existed then that is sufficient for the existence of that universal. Interestingly, this provides a defense against universals leaving existence but not against their entering existence, as it does not preclude new universals from coming into existence. I see two potential paths for Armstrong on this, neither of which he commits to but either of which could work for him here. The first would be to accept a sort of four-dimensionalism where the future is as real as the present or the past. This would handily tie up the problem of universals popping into existence, as all universals would then exist timelessly. But if he were to commit to four-dimensionalism, there would be no problem of instantiated universals entering or leaving existence at all: as future and past states of affairs would exist in an ontological sense, albeit not presently, the universals would thus be instantiated and the problem would dissolve. As he does make a defense of universals entering existence, I am not sure this is palatable for Armstrong himself, although it might be available to those with similar ontologies. Another path may be to accept a 'growing block' theory of time, where the past and present are both ontologically grounded but the future is

\footnotetext{
22 Evan Fales, "Are Causal Laws Contingent?" in Ontology, Causality, and Mind: Essays in Honour of D.M. Armstrong, ed. By John Bacon, Keith Campbell, and Lloyd Reinhardt, (Cambridge University Press, Cambridge 1993): 132-4.

${ }^{23}$ D.M Armstrong, "Reply to Fales," in Ontology, Causality, and Mind: 147.
} 
not. ${ }^{24}$ This solves the problem of new universals coming into existence: as universals are wholly present in states of affairs and new states of affairs come into existence, it is no surprise then that new universals may come into existence. ${ }^{25}$ Some evidence that this may be a path for Armstrong is present in the work of Michael Tooley, who accepts a theory of universals highly similar to Armstrong's and also a growing block theory of time. So, accepting a growing block theory of time may solve the problem of universals coming into existence and leaving existence when combined with Armstrong's thesis that a universal need only be instantiated in a state of affairs to exist.

As stated, there is no problem of similarity where objects share a universal for Armstrong, as there is no problem in a property being similar to itself. ${ }^{26}$ But by itself, this does not explain cases of similarity between distinct properties. For example, red is more similar to orange than to blue, and any two colors are more similar to each other than they are to any property of mass. Armstrong solves this problem by stating that resemblance is an internal relation between universals in virtue of their essential character. ${ }^{27}$ That red is more similar to orange than blue is a fact about the characters of red, orange, and blue and does not require an external relation between the universals to do said work. Armstrong contrasts this with cases of laws of nature, where he believes that a genuine external relation is necessary to do the work of the theory.

\footnotetext{
${ }^{24}$ C.D. Broad (1927) proposed such a theory of time.

${ }^{25}$ One may note that there may still be a problem of new states of affairs coming into existence. However, this is a general worry for growing block theories of time and not one special to Armstrong's theory of universals and as such, for now it can be omitted.

${ }^{26}$ While it is true that for Armstrong, the object is not an entity but an abstraction, the problem still arises for states of affairs as the bearers of universals. Talk of objects will still be used in this chapter for Armstrong, as part of our motivation is to view realist theories more generally through the lens of his theory, and not all realist theories reject objects in favor of states of affairs.

${ }^{27}$ Armstrong, Universals: An Opinionated Introduction.
} 
In the case of laws of nature, Armstrong tells us that a law of nature is a kind of external second-order relation between universals, and it is this law of nature that provides a grounding for scientific law-statements. Take the classic Newtonian law " $\mathrm{F}=\mathrm{ma}$ " as an example. On Armstrong's view if this is a law of nature then magnitudes of force, mass, and acceleration are all universals. The law-statement describes an actual set of relations between these universals. In this way, accurate scientific-law statements are grounded in describing something that really exists, which is perhaps the default way to ground the truth of statements about the world. Hence, Armstrong is able to provide an answer to our third desideratum.

Problems arise for this answer, however. First among these is a problem raised by David Lewis, ${ }^{28}$ in response to a problem that Armstrong addresses in his work. ${ }^{29}$ One facet of laws is that it is taken that if there is a law of nature, then the behavior of objects cannot be other than in accord with this law. In other words, the law of nature necessitates certain behavior among the particulars that instantiate it. Armstrong shies away from identifying this as metaphysical necessitation as he wishes to preserve the contingency of laws. If laws of nature are metaphysically necessary, then the intuition that laws "could have been otherwise" loses its footing. So, Armstrong says that there is a sui generis necessity present unique to laws of nature that necessitates the behavior of the objects governed by the law of nature without necessitating that those laws of nature exist. In other words, the law of nature's existence is not necessary, but given that the law of nature is present the behavior that it dictates is. Lewis points out that Armstrong needs to spell out why we ought to

\footnotetext{
${ }^{28}$ David Lewis. "New Work for a Theory of Universals" Australasian Journal of Philosophy 61, No. 4 (Dec 1983).

${ }^{29}$ Armstrong, $A$ Theory of Universals: 151.
} 
believe that this necessity exists: there must be some reason to think that the behavior of objects must be in accord with the law. As Lewis says:

But I say that $\mathrm{N}$ deserves the name of 'necessitation' only if, somehow, it really can enter into the requisite necessary connections. It can't enter into them just by bearing a name, any more than one can have mighty biceps just by being called 'Armstrong'30

Expanding on this criticism, there is a question of what laws of nature do to ensure the behavior of the phenomena to which they apply. Armstrong answers this insofar as he claims that laws of nature are both second-order universals and, simultaneously, first-order universals. ${ }^{31}$ It is notable that this is a reversal of an earlier position Armstrong took, where laws of nature were to be understood as second-order universals and second-order particulars. In being a second-order universal, the nomic relation of being a law relates the universals present in a given instantiation of a law. But Armstrong also wants to say that laws of nature are first-order universals that, like other universals, are wholly present in certain states of affairs. Namely, the states of affairs in which the law is instantiated. This means that laws are partially identical to their instances, as is true of other first-order universals. This explains how laws ensure the behavior of their instances, as if laws of nature are identical to their instances, the states-of-affairs that exemplify the laws are identical to the laws of nature, as identity is transitive. As the law is then literally a part of the state of affairs, this partial identity can ground the necessity of the law. But this creates a problem in that it is not clear what, if anything, is identical to the law in the state of affairs. Unlike universals such as the mass or shape of the object, the 'law' is only observable as a

\footnotetext{
${ }^{30}$ Lewis, "New Work for a Theory of Universals": 366.

${ }^{31}$ David Armstrong, What is a Law of Nature, (Cambridge, Cambridge University Press, 1983).
} 
regularity. If the observed regularity is the 'part' that is identical, then this is no different than other regularity theories, which Armstrong rejects. ${ }^{32}$ If something else about the state of affairs is the 'part' that is identical to the law, Armstrong needs to say more about what this part is to distinguish it from the regularity.

Armstrong may be able to provide an answer insofar as he says that causation is a relation between particulars. If all law-statements describe causal laws regarding states of affairs where one event causes another event, and if particular causal events are what ground laws of nature as first-order universals, then causal events can ground laws of nature much as particular 'mass' properties ground the universal 'mass.' I see three problems with this. First, while Armstrong does talk about causation in connection to laws of nature, he does not explicitly say that causal events are the particulars which ground first-order laws. Second, Armstrong does not state that all laws are cases of one thing causing another. His formula for a law of nature is N(F. G) where all F's are G's. This can be applied to instances which are not causal, such as F's being water and G's being H2O. Armstrong might still use causal events as a partial answer, as there might be multiple sorts of states of affairs that can ground laws of nature, with causal laws being grounded by causal events. However, this would make it more imperative to say what, beyond a regularity, makes some event or another a causal event. Lastly, Armstrong considers that there may be causal events with no associated law. ${ }^{33}$ If laws are grounded in causal events, it is hard to see why Armstrong would allow for this in his ontology, especially as he says he does not like the implications of what he terms 'singular causal events.' But he does allow

\footnotetext{
${ }^{32}$ A more thorough examination of regularity theories and their flaws will occur in the discussion of nominalism later in this chapter.

${ }^{33}$ Armstrong, "What is a Law of Nature?".
} 
that there could be a causal event without a corresponding law. This is contrasted with what he says of properties and universals, where he asserts both that for every property there is a universal and for every universal there is a property. As he does not assert this for laws of nature and causal events, and at least allows for its denial in causal events without associated laws, it does not appear that he actually asserts that laws are grounded in causal events. Which once again raises the question of what grounds causal events and brings back the earlier problems raised for his theory of how laws necessitate the behavior of their instantiations.

\subsubsection{Other Realisms}

Armstrong's is not the only form of realist answer to these problems, however. While examining his account provides us with a basis for understanding realist answers regarding properties, it may be that other views do better, or at least overcome some of the problems for the Armstrongean view. Rather than go through these views in full detail, I will attempt to contrast them with the Armstrongean view and show how they may overcome the problems in Armstrong's account as well as raise new objections that arise from accepting such accounts. ${ }^{34}$

Dretske and Tooley provide accounts of Laws of Nature in line with the Armstrongean picture, to the degree that many authors refer to this as the "DretskeArmstrong-Tooley view" or some variant on that. ${ }^{35}$ Dretske does not argue for the existence

\footnotetext{
${ }^{34}$ It should be noted that there are differences between some of these accounts that I am omitting, given the summary nature of this presentation of universals. Nonetheless, I will try to show what I take to be significant differences given the presentation of Armstrong in the earlier section.

${ }^{35}$ Notably, Armstrong (1983), Dretske (1977), and Fales (1993), among others.
} 
of universals but only that if scientific law-statements are to be anything more than mere generalizations, universals and laws of nature are needed. ${ }^{36}$ His argument is that nominalist solutions, where a law of nature is a universal truth combined with some special function that the truth serves, such as explanatory power, predictiveness, or a high degree of confirmation, is "epistemologically and functionally hopeless," and that laws as relations between universals are a viable alternative. But while this may serve to show that laws as relations between universals is a viable alternative to nominalism, it does not address alternatives that are not nominalist, such as trope theoric solutions or competing theories (which may involve universals) but do not ground laws in relations between universals, such as Bird's dispositional essentialism. $\cdot{ }^{37}$ However, it may be taken as a good critique of nominalism from a realist perspective.

Tooley's thesis is similar to Armstrong's as well, but Tooley argues for universals being transcendent rather than immanent. ${ }^{38}$ Tooley brings in this thesis to solve the problem of universals being created or destroyed. Unlike Armstrong, Tooley seems to think there is a problem with universals coming in and out of existence as long as universals are meant to be identical with their instances. If this can happen, there is nothing grounding the causal laws and relations that the property has to other properties. Tooley uses the following sort of argument for the plausibility of transcendent universals. Assume that there is some universal, $\mathrm{U}$, that has not been instantiated but which could be instantiated under the correct causal conditions. ${ }^{39}$ Describing these conditions accurately would require

\footnotetext{
${ }^{36}$ Fred Dretske, "Laws of Nature," Philosophy of Science, Vol 44, No. 2 (Jun 1977)

${ }^{37}$ Alexander Bird, "The Dispositionalist Conception of Laws", Foundations of Science, Vol 10 (2005)

${ }^{38}$ Michael Tooley. "The Nature of Laws", Canadian Journal of Philosophy, Vol 7 (1977)

${ }^{39}$ This is a plausible modal claim even on a four-dimensionalist picture, as there does not seem to be any guarantee in physics that each property will necessarily be instantiated somewhere at some time.
} 
referring to $\mathrm{U}$, and presumably any law regarding the instantiation of $\mathrm{U}$ is a true law of nature that is reflected in however we ground causal relations. Yet, as U is never instantiated, there is no object in the world to enter into the causal relations in question if $U$ only exists where it is instantiated. However, if U (and other universals) are transcendent, this problem abates. Transcendent universals may solve these problems but come with their own baggage. Recall that Armstrong solved the problem of how relations between universals govern the behavior of states of affairs by the imminence of those universals: the fact that the universal is fully present in the states of affairs that instantiate it is what explains how laws regarding that universal govern those states of affairs, and why relations between universals hold for their respective particulars. As Tooley's universals are transcendent, they are not wholly present in the states of affairs which instantiate them as are Armstrong's. Instead, Tooley says that there is a relationship between universals and their particulars wherein facts about universals logically necessitate generalizations about the particulars to which they correspond. ${ }^{40}$ As Tooley says:

"This idea of a statement about particulars being entailed by a statement about a relation among universals is familiar enough in another context, since some philosophers have maintained that analytical statements are true in virtue of relations among universals."

But I would argue that this is insufficient: that an idea is familiar is not enough to argue for its truth. Nor is it sufficient that 'some philosophers' have maintained it. But even if we take the statement that 'analytical statements are true in virtue of relations among universals' as given, this still does not show how or why relations between transcendent universals

\footnotetext{
40 Tooley, "The Nature of Laws."
} 
would influence the behavior of particularized instances of those universals. In Armstrong's account the need for such a mechanism by which relations between universals inform the behavior of states of affairs which instantiate them is mitigated by virtue of the fact that those universals are wholly present where they are instantiated. But as Tooley's universals are transcendent, they cannot govern the behavior of particulars in this way. Tooley might want to make the stronger claim that he seems to imply here that there is a relationship of necessity akin to the truth of analytical statements between relations between universals and the behavior of particulars. He could make the argument that as analytical statements can be made true in virtue of relations between universals because of the essential character of those universal the behavior of particulars can be explained by the essential character of those universals which are present in those particular instances. But this sort of necessity would require a decisive argument rather than a mere suggestion, which I have not been able to find in Tooley's work. At minimum, such an argument for a necessary connection of relations between universals governing the behavior of the particulars which instantiate those universals would need to be made for this theory to be viable.

Like Tooley, Evan Fales argues for universals existing as transcendent entities. However, Fales takes the argument a step further. Tooley does not explicitly argue for the existence of uninstantiated transcendent universals although his argument certainly implies their possibility. Fales, by contrast, argues that if uninstantiated universals are plausible they may be likely given the potential likelihood of unrealized causal relations. ${ }^{41}$ Fales further differs from Armstrong's view as Fales takes laws of nature not to be grounded in relations between higher-order universals, but in dispositions that are

${ }^{41}$ Evan Fales, Causation and Universals, (Routledge, New York, 1990): 148-65. 
inherent to physical universals. As the disposition is a part of the essential nature of the universal, Fales holds that the necessity of laws is metaphysical necessity. This further differentiates him from Armstrong, whose view is that laws have a sui generis necessity. This is meant to solve the problem Lewis brought against Armstrong by more tightly linking the necessity of causal laws to the properties to which they apply. But this results in two problematic consequences. The first is that it runs counter to intuitions about the contingency of laws: namely, that they could have been otherwise. Given that laws are grounded in dispositions of universals and that those dispositions are essential to that universal being the kind of thing that it is, there could not be different causal laws without different universals. To use the example of " $\mathrm{F}=\mathrm{ma}$ " as a law again, if $\mathrm{F}=\mathrm{ma}$ describes a law of nature than, on Fales' view, " $\mathrm{F}=\mathrm{ma}^{2}$ " is impossible. For Fales, causal laws describe causal relations. These are second-order relations, the relata of which are types of events. To be a type of event is to be a universal, and it is inherent in the nature of that universal that it has the causal relations that it does. So, $\mathrm{F}=$ ma describes a relation between universals, and it is a necessary feature of those universals that they have the relations that they do. Fales, for his part, seems unperturbed by this, stating in many places that he finds the intuition about the contingency of laws suspect and that he believes, in the end, that this intuition ought to be abandoned. ${ }^{42}$

There are other realist accounts which, while they admit universals into their ontology, differ from Armstrong's more significantly. For instance, Lowe's 4-category

42 Ibid. 
ontology ${ }^{43}$ admits several kinds which Armstrong's does not while Bird ${ }^{44}$ attempts to ground laws of nature in a universal's having a disposition rather than in external secondorder relations between universals. While such theories are relevant for the current debate, I do not have space to describe them here. I have focused on Armstrong's account and those that have built upon it both because of its influence in the current philosophical debate and because I take it to be a plausible realist account. However, I acknowledge that there are non-Armstrongean realist accounts that I have not done justice to in this work.

\subsection{Nominalist Theories}

Having looked at realist theories, we can turn our attention to their chief opponent historically speaking: nominalist theories. But there are several difficulties in analyzing nominalist accounts. The first is in disputes about what qualifies as a nominalist theory. For instance, Armstrong claimed (and others have repeated) that Quine held a nominalist theory. ${ }^{45}$ But Quine himself disputed this, saying not only that he believed in universals but that, "I see no way of meeting the needs of scientific theory, let alone everyday discourse, without admitting universals irreducibly into our ontology." 46 Armstrong rejects this because he does not think that Quine's classes are genuine universals. For myself, I tend to take philosophers at their word for what they think they are committed to and if Quine says he is committed to the existence of 'platonic entities,' as he does, then I am inclined to take him at his word that his is not a nominalist. A further difficulty lies in the fact that

\footnotetext{
${ }^{43}$ E.J. Lowe, The Four-category Ontology: A Metaphysical Foundation for Natural Science, (Oxford, Clarendon Press, 2005).

${ }^{44}$ Alexander Bird, Nature's Metaphysics: Laws and Properties, (Oxford, Clarendon Press, 2007).

45 David Armstrong, "Against Ostrich Nominalism," Pacific Philosophical Quarterly 61 (1980).

${ }^{46}$ W.V. Quine, "Soft Impeachment Disowned," Pacific Philosophical Quarterly 61 (1980): 450-1.
} 
nominalisms vary more widely than realist theories do. While realists have a unifying posit in that they accept the existence of multi-present properties, nominalists only have in common that they reject the existence of properties in favor of other ways of solving the problem of universals and accounting for the appearance of properties. Armstrong himself attempts a taxonomy of nominalisms and comes up with five categories: predicate nominalism, concept nominalism, class nominalism, mereological nominalism, and resemblance nominalism. ${ }^{47}$ Thus, finding ways to properly criticize a wide diversity of nominalist views and properly addressing the individual philosophers who hold them would take a much larger work than this. Finally, while nominalists do give accounts of how to ground scientific laws, many (if not most) reject that these laws are grounded ontologically, instead preferring a pragmatic or epistemic ground for scientific laws. Thus, as many nominalists reject the third desideratum defended here, examining how successful they are in answering that desideratum is problematic.

So, while acknowledging the problems with representing a diverse array of theories with a single instance as I did with Armstrong, I will attempt to do so and hope to come to some more general criticisms which may be applied to a nominalist theory. Thus, I will examine David Lewis' nominalist account. The reasons for choosing Lewis are several. First, he regularly engages with Armstrong and other realists about laws of nature and directly answers the desiderata defended at the end of Chapter 1 . Second, his account is detailed and well-developed and broadly influential. Lastly, while it is certainly the case that his possible worlds realism is considered somewhat idiosyncratic, the best-systems analysis

\footnotetext{
${ }^{47}$ David Armstrong. Nominalism and Realism: Universals and Scientific Realism Volume 1, (Cambridge, Cambridge University Press, 1980).
} 
that follows from it gives us an idea of what a nominalist account which ontologically grounds the truth of scientific laws would look like. As such it shows that a nominalist may still ground scientific laws ontologically.

\subsubsection{Lewisian Objects and Possible Worlds}

Unlike Carnap, Lewis' commitment to nominalism is at best half-hearted. Lewis does not take it that universals would be a bad admission to his ontology, and even considered publicly whether he ought to admit them. ${ }^{48}$ Still there are reasons to take his ontology to be nominalist in nature. For one, while he notes the he could solve problems for his theory by admitting universals ${ }^{49}$ he does not go so far as to actually accept them. Further, the problems he takes universals to have are significant. He thinks that structural universals are so difficult as to be untenable, while recognizing that a theory of universals requires them to explain the similarity of things with common structures. ${ }^{50}$ Moreover, Lewis claims that his realism about possible worlds, combined with accepting set-theoretic constructions of individuals, gives one all they need to explain the nature of properties. ${ }^{51} \mathrm{By}$ accepting realism about possible worlds, one commits to the word 'actual' being merely indexical: possible worlds are just as real as the actual world on this view, and the only thing that distinguishes the actual world is that it is the one in which we find ourselves. So even if Lewis himself was not a pledged nominalist, his view is best described as a nominalist view.

\footnotetext{
48 Lewis, "New Work for a Theory of Universals."

49 Ibid.

50 David Lewis, “Against Structural Universals,” Australasian Journal of Philosophy 64, No. 1, (March 1986)

51 David Lewis, On the Plurality of Worlds, (Oxford, Blackwell Publishing, 1986).
} 
To begin with, we will grant Lewis his theory of possible worlds. While this theory is contentious, Lewis' claim is that if we give him this theory he can explain the nature of properties. So, we will grant him this theory for the sake of argument. Lewis says that once one accepts possible worlds, properties can be analyzed in terms of classes of objects wherein to have some property is then to be a member of a particular set. A problem for this sort of theory noted by Armstrong and others is that classes may be coextensive. In other words, more than one property may be identified by the set. ${ }^{22}$ For instance, take the set of living organisms with hearts (cordates) and the set of living organisms with kidneys. (renates) If every creature with a heart also has a kidney and vice versa, then the set of renates and cordates are identical. Yet to have a heart is not to have a kidney. Lewis is aware of this, however, and says that by including not merely the actual objects but the possible ones, no such overlaps will occur unless there is a necessary connection between the properties. To see how this solves the problem, imagine it is necessary ${ }^{53}$ that every creature who has a heart has a kidney and vice versa. Then to be a renate is necessarily to also be a cordate. But if it is not necessary that two predicates be coextensive, then it is possible $^{54}$ that some creature is a renate and not a cordate or vice versa. As possibilities are all actual at some world for Lewis and as our classes extend over all possible worlds, there would then be a member of the one set that is not a member of the other, meaning they are not coextensive. In this way, Lewis gets past this difficulty for class nominalism: for all coextensive predicates, either they are necessarily coextensive or they fail at some possible

\footnotetext{
${ }^{52}$ Armstrong, Nominalism \& Realism: Vol. 1.

53 The species of necessity, for Lewis, will impact the 'closeness' of the possible worlds in the set. If we wish for it to be the total set of possible worlds, imagine that the necessity is logical necessity.

${ }^{54}$ Again, the species of possibility will depend, for Lewis, on the closeness of the world: it might be physically possible for something to be a renate but not a cordate, but not biologically possible, for instance.
} 
world. ${ }^{55}$ This also provides an answer to our second desideratum, as similarity between objects is then explained in terms of class membership: two objects are similar with respect to some property if they are a member of a shared class.

But while the classes that define properties for this Lewisian picture exist across worlds, the grounding of scientific law statements is not. Lewis defends a view of Humean supervenience wherein laws are present at a world ${ }^{56}$. Lewis takes his view from Ramsey, who put it in these terms:

"I, therefore, put up a different theory by which causal laws were consequences of those propositions which we should take as axioms if we knew everything and organized it as simply as possible in a deductive system." 57

This view, termed the 'Best Systems Analysis' in the literature, is that laws of nature are neither governing entities nor mere descriptions. Imagine we set out to make a series of statements that if taken as axioms would describe all events within the world, either directly or by implication. There exists an infinite number of classes of statements we could consider for this purpose. For each set there are three relevant, competing criteria we may consider: the simplicity of the system in question, the completeness of the system in question, and the accuracy of the system in question. As Lewis explains it:

"Take all deductive systems whose theorems are true. Some are simpler, better systematized than others. Some are stronger, more informative, than others. These virtues compete: an uninformative system can be very simple, an unsystematized compendium of miscellaneous information can be very informative. The best system is the one that strikes as good a balance as truth will allow between simplicity and strength.

\footnotetext{
55 This is Armstrong's term, not Lewis', but the classification is useful for our purposes.

${ }^{56}$ David Lewis, "Humean Supervenience Debugged," Mind, Vol 103, No. 412 (1994)

${ }^{57}$ Frank Ramsey, The Foundations of Mathematics and other Logical Essays, (London, Routledge, 1931).
} 
How good a balance that is will depend on how kind nature is. A regularity is a law iff it is a theorem of the best system." ${ }^{58}$

The best systems analysis (BSA) view is that at a world, there is some set of statements that will provide the best balance of simplicity, completeness, and accuracy such that it may serve as the laws of that world.

A question then arises with respect to our third desideratum: what is the ontological grounding of laws? If laws are merely classes of propositions, the most obvious answer is that thinking beings ground such laws as the entities thinking of the statements. But Lewis rejects this. ${ }^{59}$ For Lewis it is not required that a being at a world think of the laws of a world for those laws to exist. It is instead a modal claim: if a being were to form a set of axioms at a given world with the greatest combination of simplicity, completeness, and accuracy then that would be the set of laws. As Lewis is a possible worlds realist this means that he believes that there is in fact a world where someone has done just this. Lewis likewise rejects the notion that completeness, accuracy, or simplicity are merely features of our psychology: there is a reason, he insists, that we find one system of axioms to be simpler than another: that it is actually simpler. ${ }^{60}$ The ontological basis for scientific law-statements is then two-fold. First, the actual objects of the universe form the basis for which system forms the best axioms at that world, and second, that there is a being, at that world or at

\footnotetext{
${ }^{58}$ Lewis, "Humean Supervenience Debugged."

59 Ibid.

${ }^{60}$ Lewis does not say whether he thinks these standards are rooted in logic or something else. If rooted in logic, they will be the same across worlds while if they are not, they will vary by possible world. I take the former to be kinder to Lewis, so I will use that from here on out.
} 
some other world, that describes the world using that system of axioms in a way that is objectively better than any other set.

With respect to our fourth and fifth desiderata one might intuitively think that Lewis' story does very well: after all, simplicity and strength are already a part of the best systems analysis of laws. However, that Lewis applies similar criteria in grounding scientific law-statements does not mean that his analysis does well by our desiderata. And one may think that his method does not do well. For one, Lewis does not explicitly rule out universals and even considers that accepting at least some universals may solve problems for his theory. ${ }^{61}$ This would make his account accept at least as many kinds of entities as Armstrong's does. Additionally, there is the problematic addition of possible worlds to Lewis' ontology. Lewis is aware of this objection and claims that this does not add complexity to his account. ${ }^{62}$ Lewis says that while there are quantitatively more entities in his account these are the same kinds of entities: individuals and worlds. For Lewis' account, actual and possible entities are of the same sort, with 'actual' serving merely as an indexical. So he claims there is no problem of increased complexity.

There is an interesting issue here with respect to whether or not worlds are individual entities akin to the objects that inhabit those worlds or if they are another kind of entity. If they are a wholly different kind of entity, this may be another mark against Lewis, as there are other views do not explicitly make use of worlds in their ontology. I believe Lewis can answer this by showing that neither horn of the dilemma is a problem for him. If worlds are individuals he has a one-category ontology. If worlds are not individuals

\footnotetext{
${ }^{61}$ Lewis, "Against Structural Universals."

${ }^{62}$ David Lewis. Counterfactuals, (Oxford, Blackwell, 1973).
} 
but a wholly different kind of entity then other views need to make use of at least one world existing: the actual world. The only alternative would be to present an ontology without the actual world, but without an actual world it is not even clear what there is for ontology to explain. If every view needs to assume the existence of worlds to get off the ground then Lewis' view is no better or worse than any other in this regard.

Here I disagree with Lewis, as in most other views the assumption of a world does little to no work for the theory. Realists and even nominalists do not have the existence of a world do any heavy philosophical lifting. For Lewis possible worlds do almost all of the philosophical lifting. There is a significant difference in theory evaluation between a theory that does not deny the existence of an entity and one that makes use of an entity. If a metaphysical view admits that dogs exist, this assumption may be harmless. If it puts all of its weight on the existence of dogs, suddenly the assumption of the existence of dogs is of great importance. Similarly, if possible worlds pulled no real philosophical weight we might not have reason to admit them into our ontology but neither would anything ride on rejecting or accepting them. But given the weight that is put on them in Lewis' account there is more at stake in assuming their existence than other philosophers have with respect to the assumption of an actual world.

Further, I find Lewis' method of parsing simplicity suspect. Rather than talk of qualitative or quantitative simplicity, I think the distinction is between theoretical and observational entities and kinds, as I defended in the earlier discussion of desiderata. Lewis makes this same division, perhaps without meaning to, in his own justification for his qualitative vs. quantitative simplicity: 
"A doctrine is qualitatively parsimonious if it keeps down the number of fundamentally different kinds of entity: if it posits sets alone rather than sets and unreduced numbers, or particles alone rather than particles and fields, or bodies alone or spirits alone rather than both bodies and spirits. A doctrine is quantitatively parsimonious if it keeps down the number of instances of kinds it posits; if it posits $10^{29}$ electrons rather than $10^{37}$, or spirits only for people rather than spirits for all animals." 63

While these examples show his qualitative vs. quantitative simplicity, they also show a division between theoretical and empirical entities. Positing sets alone rather than sets and unreduced numbers posits fewer theoretical entities. Positing spirits and bodies posits more kinds than either alone but restricting spirits to people does not. To show why this is, take the existence of Earth. We need not worry about whether or not we have theoretical justification to assume Earth exists as this is a matter of observation and not merely one of theory. Now take the theory that on the opposite end of the sun, carefully hidden from our view, is an alternate Earth. Without empirical confirmation we may reject this theory on the grounds of parsimony: while we might not be able to observe such a planet, we can reject the theory on its lack of simplicity: it adds an entity where we need not assume one exists. In fact, theories such as hollow earth theories, extraterrestrial abductions, and 'Planet X' theories are rejected on this basis. While the theories may not posit any entities that are of a different kind than the entities we are aware of, they unnecessarily posit additional entities to explain a phenomenon that can be explained without them, and these theories may still be rejected as unparsimonious. We accept the existence of theoretical entities, particularly in the sciences, when accepting them gives us a more robust explanation of some phenomenon. In typical cases, merely quantitatively different entities are

${ }^{63}$ Ibid. 
presumably observational: there is some difference in the world between a world that has those entities and one that does not which could, in principle, be verified. Lewis' possible worlds are not like this. While they and the entities that reside in them are quantitatively similar to our own world, they are not empirical entities: they are posited only to fill a theoretical gap. As such they are only to be accepted if we cannot explain some phenomenon without either postulating them or postulating some yet-more complex explanation.

There are significant issues that arise with Lewis' account with respect to the fifth desideratum as well. But as what we are after is a more general critique of nominalist accounts, rather than to enumerate those issues which may be idiosyncratic to him, I will look for common threads that may then be extended to other nominalist accounts.

\subsubsection{Problems of Nominalism}

Before getting into the difficulties that arise from accepting a nominalist account like Lewis provides, we should examine how well they have done with respect to our other desiderata and bring some general worries to the table. Right away, we might worry that they have failed with respect to the first desideratum Lewis does not preserve properties as entities at all. If the goal was to explain how an object can have a property, one may presume that properties are a necessary part of the account. But Lewis tries to explain away properties: that is, he tries to reduce or eliminate properties from his ontology by explaining why it is that objects appear to have properties. This is, I think, acceptable. When we explain heat as being mean kinetic energy, we affirm that there is no independent 'heat' in the world: we say that when we discuss heat, we are discussing mean kinetic energy. 
Similarly, when Lewis or other nominalists talk about properties they explain them away by reducing or eliminating them. We may rightfully ask how well they do with this, but the strategy is in general a sound one provided that properties are not somehow snuck back in.

For our second desideratum, Lewis does an adequate job of explaining similarity, which should be unsurprising. Even opponents of Nominalism such as Armstrong acknowledge that a class nominalism such as Lewis' is a strong candidate for handling the problem of universals. Further, his use of possible world realism allows Lewis to overcome some difficulties a nominalist might otherwise have. Take the following criticism of Armstrong's: that classes and types are not one to one. Armstrong gives this example: "First, the Class Nominalist requires that corresponding to each type (kind, sort, property), there is a class. That seems fairly right... A bit more worrying are types to which no instances correspond: unicornhood and centaurhood, for example. Set theory does recognize the null class. But the trouble is that there is only one null class. If being a unicorn is identical with being a member of the null class and being a centaur is identical with being a member of the null class, then, by the transitivity and symmetry of identity, being a unicorn and being a centaur are the very same type. This seems absurd."

So, a problem for the class nominalist involves type instances that belong to a null class. But for Lewis, the only classes which correspond to the null class will be those classes which are null across all possible worlds. Unicorns and centaurs are not impossible creatures, even if they are not actual at our world, and so Lewis will not have these classes correspond to the null class. There will be classes which correspond to the null class: those which include only impossible things: round squares, true falsehoods, and the like. While it may still seem absurd that different impossible types are identical with each other, the effect of this criticism is at least diminished for Lewis since all of the things mentioned cannot be 
realized at any world. Unlike centaurs or unicorns, there isn't a character of a round square that we can make sense of. So, it is more plausible for these classes to be identical while answering the problem of universals adequately.

But Lewis may have problems with respect to our third desideratum and his best systems analysis of laws of nature. This problem is that his laws of nature ended up grounded not in the state of the world but in the mental states of thinking beings. This makes his account fall into a sort of psychologism about laws of nature. Lewis attempts to avoid this by using Ramsey's conditional, 'if we knew everything and organized it as simply as possible in a deductive system.' However, this runs afoul of Lewis' modal realism: if it is possible for something to fulfill a conditional, then in some world, it does. So, the account appears to be psychologically grounded after all, as the possibility is grounded in the existence of an entity which at some world conceives of the theory, rather than grounding it in the entities the theory references. Lewis may choose to accept the psychological account here, or he may reject it. If he rejects it, he must declare such a being impossible as if it is possible it will exist at some world. Further, in choosing this route, Lewis has no possible ontological ground for a best systems analysis. If a best systems analysis is literally impossible but also determines how we are to understand laws of nature, then it seems we must accept nihilism about laws. I think that presented with these paths, the former is initially easier for Lewis, but this route has its own difficulties.

So, Lewis might want to avoid this by taking the psychological approach. As I think this is a route that may be attractive to nominalists more generally, I want to address it here in more detail. The key problem I see with this is error: conscious beings can make judgements about reality that are false. I can think my keys are at home when I left them in 
my car, I can think that heat is phlogiston when it is really mean kinetic energy, and I can think the world is flat when it is actually round. Whether it is a matter of memory, perception, or judgement, conscious beings err. By making one's ontological criteria revolve around the psychology of conscious beings, one invites errors of judgement into one's ontology. Referring to a 'typical' observer or person or agent does not help here: typical observers make errors regularly. A typical person at Houdini's show may see him make an elephant disappear, but we do not think this in and of itself means that he made an elephant cease to exist. It seems that what is needed is an ideal conscious person but defining such a person creates problems.

Lewis may be able to avoid this by something like the following. We know from experience that a world may contain agents within it. Those agents can form propositions and take them to be axioms in a deductive system. One of these deductive systems, when applied to a world, will constitute a best systems analysis of the laws of that world. Ergo, for each world there is a best systems analysis of that world. Even if no agent at that world (or our world) forms the best systems analysis for a particular world, as it is possible for an agent to do so, we may say that there is such an analysis in the same way that an unsolved problem may still have an answer. Of note, if this strategy works and gets Lewis past this problem, it might also be available to other forms of nominalism. This would have the interesting consequence of nominalists who fall into some form of psychologism being able to save their view by means of possible world realism.

There is at least one reason to doubt the possible worlds solution for the nominalist with respect to psychologism, which I will bring to bear here. In order for BSA to work at a given world, there must be some system with the best mix of accuracy, simplicity, and 
strength for that world. This means that the world must in some sense be conceivable: it must be possible for some entity to conceive of the laws of that world. As the possible/actual distinction is merely indexical for Lewis, if BSA works at every world then this means that for every world there exists some conscious being (in some world) who is both capable of and in-fact has conceived of a BSA for that world. This means that there cannot be any world such that its laws cannot be conceived of by some agent in some world. The possible worlds solution to psychologism thereby requires that there be no 'inconceivable' worlds. So do any such worlds exist? I know of no proof that says such worlds exist or that they do not. But it seems an extraordinary claim that every possible world has a BSA that is conceivable by some being. At minimum, this requires a proof.

I believe there are also reasons of simplicity to be suspicious of possible worlds, as established earlier. Given that our primary concern here is general criticisms of nominalism rather than possible worlds realism, this may seem to be a bit of a digression. However, as shown above, one way of avoiding the problems for psychologism about laws of nature for the nominalist is to accept possible worlds realism. If a nominalist is to accept a psychological ground for scientific laws then an answer such as possible worlds realism may be required. For the nominalist who does not want to accept possible worlds, an answer which does not run into problems of simplicity or undue philosophical baggage will be required. ${ }^{64}$ Lewis' possible worlds realism, for many philosophers, constitutes a burden on their ontology that they are unwilling to bear while creating problems with respect to a simplicity criterion, and nominalists who wish to advance theories which do similar work

\footnotetext{
${ }^{64}$ As stated earlier, this is not sufficient to show that all forms of nominalism have this problem, but such an investigation is beyond the scope of this work.
} 
will need to find something to take the place of possible worlds in their ontology to avoid the problems of psychologism in their ontology.

\subsection{Trope Theories}

Having examined realist and nominalist accounts of properties and the ontological grounding of scientific law-statements, we will move on to trope theories. A trope theory admits the existence of properties but does not admit them as multi-present objects. Given that the theory I will ultimately come to defend is a sort of trope theory, I will pay special attention to the trope theories examined so as to better explain the benefits and problems

for the trope theorist. I will be examining two trope theories: Keith Campbell's bundle theory and C.B. Martin's substrata theory. I chose these for two reasons. First, each explicitly answers the questions posed in our first three desideratum. Second, for the sake of examining trope theories I find it useful to examine both a bundle theory and a substratum theory, as most trope theories will be one or the other. While both have their advantages, I will argue that Campbell's theory does not adequately account for scientific laws, while Martin's theory brings in substratum unnecessarily. Ultimately, the theory I will propose will use elements of both theories to provide a single-category ontology that can ground scientific law statements with little in the way of excess philosophical baggage.

\subsubsection{Campbell's Bundle Theory}

Campbell holds a bundle theory of tropes. This means that all objects in his ontology consists of bundles of tropes, and that tropes make up the only category in his ontology. Relations, events, causes, etc. will all be either tropes, or not admitted into his ontology. 
Campbell holds that objects are nothing over and above spatio-temporally co-located (compresent) tropes. ${ }^{65}$ Before moving forward, I would like to examine one untoward consequence of the bundle theory, how Campbell falls prey to this consequence, and something of how he may be trying to resolve this dilemma.

\subsubsection{Necessity of Object Composition}

For bundle theories such as Campbell's, being an object is nothing over and above being a collection of compresent tropes. As Campbell says:

Think of a diamond. The trope theory of diamonds is a bundle theory. This diamond is a compresent bundle of tropes, i.e. of particular cases of qualities. It combines in a compresent collection hardness, transparency, brilliance, many-facetedness, a carbon constitution, an inner crystal lattice, inner electro-magnet and other sub-atomic forces, mass, solidity, temperature, and so on. ${ }^{66}$

This answer of what it is to be an object is typical of bundle theories. But it carries with it an untoward consequence. If each object is just a collection of individualized tropes, then it is necessarily true of that object, if not the kind in general, that it has the collection of tropes that it does. Take the shape of a particular diamond. Call this trope S. S is a particular trope in the bundle of its particular diamond. To be that diamond is nothing over and above being the particular collection of compresent tropes that includes S. As such, it is an analytic truth that the diamond in question possesses each of its tropes, including its shape. It could not have a different shape, or it would be a different collection of compresent tropes and hence, a different object. One might think this is a problem only if we have the diamond change properties, prompting questions such as, "If the diamond's shape changes, is it the same

\footnotetext{
${ }^{65}$ Keith Campbell, Properties.

${ }^{66}$ Campbell, Properties, p. 20.
} 
diamond?"67 But it runs deeper than this, because it grants an analyticity to the propertyobject relationship that we do not generally uphold in other contexts. It makes no sense to even talk about the diamond having another shape on this view. If it had another shapetrope, it would be a different diamond altogether. One might attempt to ameliorate this by moving to something more akin to Simon's nuclear theory of tropes, ${ }^{68}$ or to otherwise distinguish between those tropes held accidentally and those tropes held essentially by an object. This ameliorates the problem but does not entirely eliminate it. For even on the nuclear theory an object has certain essential tropes, and that a particular object has the particular essential tropes that it has is a necessary truth. If the hardness of a particular diamond is one of its essential tropes, then it could not be the same diamond or even the same object unless it had that same hardness trope. Any 'swapping' of tropes, ${ }^{69}$ including indistinguishable tropes, would produce a different diamond. This may be unobjectionable as one may argue that this is what it means for a property to be an essential property for an object. However, Campbell not only does not make this distinction but explicitly uses the argument that a change in indiscernible tropes would produce different objects in his response to objections to the trope theory..$^{70}$ Yet even in the less objectionable case, rendering any true statement of the form "Object X possesses property Y" necessary is still a bullet to be bitten, and one that Campbell seems ready to bite. Campbell does still attempt

\footnotetext{
${ }^{67}$ As we will see, Martin raises the problem in this way.

68 Peter Simons, "Particulars in Particular Clothing," Philosophy and Phenomenological Research 54, No. 3 (1994): 576.

${ }^{69}$ Whether or not tropes can be 'swapped,' as well as the modal status of the 'can' here depends largely on one's account of change in a trope ontology.

${ }^{70}$ Campbell, Properties, p. 72.
} 
to provide an account for change in properties, although these properties will not turn out to be true tropes, which I will examine when we get to his field theory.

\subsubsection{Resemblance and the Trope Theory}

Campbell begins his account of similarity by giving us an analysis of predication under a trope theory. ${ }^{71}$ Take the phrases, "This electron has a negative charge," and "All electrons have a negative charge." Williams ${ }^{72}$ analyzes such phrases in the trope theory as translating to, "This electron is a case of a thing with a negative charge," and "Every electron is a case of having a negative charge." Campbell claims that using this sort of translation, all issues of predication are issues of what he terms 'self-predication.' But how is it that these different charges resemble? Campbell makes the case that even for the realist, two items can resemble in ways other than by possessing a common universal. He does this by introduction of the Third Man problem. Take the universal 'humanity'73 and an individual in whom this universal inheres, Socrates. Socrates and humanity must resemble, so the problem goes, as Socrates is a case of humanity. ${ }^{74}$ If that which instantiates a universal fails to resemble that universal, it is not clear how there could be any resemblance regarding universals at all. But if resemblance is always due to sharing some universal, then the universal humanity and the individual Socrates must share some third universal, which then itself must resemble humanity and Socrates, spawning an infinite

\footnotetext{
${ }^{71}$ Ibid:. 42.

72 D.C. Williams, The Ground of Induction, (Cambridge, Harvard University Press, 1947).

${ }^{73}$ This problem does not depend on the realist accepting 'humanity' as a universal and can be created using other universals a realist is more prone to accept.

${ }^{74}$ Campbell does not tell us how it is that humanity and Socrates resemble, but instead argues that it must be so: that any coherent version of resemblance must admit that there is some manner in which Socrates resembles the universal 'humanity' if Socrates is to somehow instantiate it.
} 
regress. Campbell says that a promising path is to say that part of Socrates shares partial identity with humanity. ${ }^{75}$ In other words, Socrates' individual instance of the property 'humanity' is identical with the universal humanity in part. This part is humanity as it exists in the instance of Socrates. This 'partial identity' here means that it is not the case that the entirety of the universal is present in each of its instances. Instead, it means that there is a property which the object possesses, and that this property is shared commonly between the object and the universal. The universal, in virtue of having instances, has 'parts.' This accounts for the similarity between Socrates and humanity without creating a 'third man.' I do not find this especially persuasive as realists such as Armstrong have shown that it is possible for a realist to avoid this problem by avoiding the issue of 'partial identity' of universals entirely. If the universal is wholly present in its instances, then there is no distinction between the 'humanity' of Socrates and the universal 'humanity': the two are one and the same property. I believe this neatly avoids the regress, as I take the fact that a property exactly resembles itself to be an ontological free lunch. I do believe it is a good reason to reject a theory of universals which postulates partial identity between a universal and its instances, but these sorts of considerations are why Armstrong himself rejects this position. ${ }^{76}$

Nevertheless, Campbell uses this objection to open the door for a trope theory of resemblance. Campbell does not provide an analysis of resemblance in terms of partial identity or any other, more basic phenomenon. Instead Campbell takes resemblance to be a

\footnotetext{
${ }^{75}$ As we have seen, Armstrong and certain other realists have taken a different approach and have instead said that the universal is wholly present in each instance, rather than merely partially identical to each instance.

${ }^{76}$ Armstrong, Universals: An Opinionated Introduction.
} 
primitive of his theory. Particularly in comparison to the realist, who does give an account of what it means for two objects to resemble, this creates a problem for Campbell's theory. If part of the problem of universals is to explain similarity between objects in the world, and Campbell recognizes that it is, ${ }^{77}$ then taking resemblance as a primitive causes his theory to fall short of that goal. Resemblance and similarity are mere synonyms and if Campbell takes resemblance to be a primitive he is not offering an explanation for how it is that two things resemble. While philosophers are free to choose their primitives, calling something a primitive is not the same as providing an explanation. To explain how a bird flies is different than simply taking it as a fact that they fly. As the realist has an explanation of resemblance in terms of partial identity and even certain nominalist theories can explain resemblance between objects without resorting to using resemblance as a primitive, Campbell's theory cannot rely on their being no such alternatives. As part of what we set out to explain is how it is that two things resemble, this means that Campbell's explanation falls short. Perhaps Campbell could claim that realist and nominalist theories carry with them too many collective problems in attempting to analyze resemblance and so taking it as a primitive is our only recourse. But while Campbell presents many problems for realists and nominalists alike he fails to give a demonstration that shows that any analysis of resemblance is to be problematic. And so with respect to our second desideratum, Campbell's view is weaker than others presented.

${ }^{77}$ Campbell, Properties, p. 28 


\subsubsection{Scientific Laws and the Bundle Theory}

So what of our third desideratum then? Campbell denies that truthmakers of scientific-law statements provide any form of necessitation. Rather, his path is entirely Humean. Campbell claims that laws of nature only provide an account of "what is immune from human manipulation." 78 Laws of nature are important on his view: they state something about the basic, inner workings of an object given some set of its properties. But those basic, inner workings might have been different, he argues, and so there is no special necessitation required to explain them.

But this leaves an open question for Campbell: could the laws of nature be different and yet the properties of objects be the same? This is difficult to settle for Campbell, but we can do so if we ignore the case of probabilistic laws and consider two worlds whose objects only possess deterministic laws. Consider two worlds, w1 and w2, such that all causal processes that occur within those worlds are deterministic. At times $t_{n w 1}$ and $t_{k w 2}$, respectively, $\mathrm{w}_{1}$ and $\mathrm{w}_{2}$ exactly resemble, meaning that for each world there is a counterpart in the other that exactly resembles it and all relations between counterparts in those worlds also resemble, along with any other sundry metaphysical parts that may need to resemble each other. After $t_{n}$ and $t_{k}$, respectively, can $w_{1}$ and $w_{2}$ not resemble? If so, it can only be because of different causal results in $w_{1}$ and $w_{2}$ which happened in spite of

\footnotetext{
${ }^{78}$ Campbell, Properties, p. 75.
} 
having deterministic causal processes and all of their objects resembling exactly. ${ }^{79}$ Recall that for someone like Lewis, this result is possible. ${ }^{80}$

For Campbell this is not possible. Campbell examines the case of a soporific having the power to induce drowsiness. ${ }^{81}$ Campbell says that to understand how a thing has the causal powers that it has, we must examine its inner workings. Causal powers thought of in this way are not bare facts: a soporific does not induce drowsiness by some opaque 'causing drowsiness' power, but by having complex, contingent powers that involve the interactions of its molecules with the human nervous system. The 'contingency' is presented in two different ways. First, different objects which share a natural kind may not have the same causal powers. Campbell offers the example of a breed of opium that is non-soporific. The second way is that causal powers only furnish the 'causal tie' under appropriate circumstances. If someone's neural structure is not appropriately receptive to opium, for instance, it will not act as a soporific when given to them. In this way Campbell distinguishes between the basis for a causal power and the power itself. The causal power itself is only present when it is manifested, but the basis for that causal power exists throughout. As a bundle theorist, the basis for this causal power will always be some trope. Provided that tropes that form the appropriate causal basis are in the proper circumstances, then they will produce their causal effects. But given the number of

\footnotetext{
${ }^{79}$ This is why the qualifier of deterministic causal laws was necessary for the case. Campbell allows for probabilistic causation: causal processes whose results only manifest probabilistically. This makes the case substantially more difficult to formalize in a Humean manner without specifying what it means to be a probabilistic causal manifestation in a Humean system. The deterministic case is easier to understand and was thus utilized here.

${ }^{80}$ David Lewis, On the Plurality of Worlds.

${ }^{81}$ Campbell, Properties: 119.
} 
potentially intervening factors, Campbell refuses to grant any sort of necessity to this fact. It is these causal powers that ground laws of nature, for Campbell. ${ }^{82}$

I agree with Campbell on much of this. Yet it seems to me there is a hidden form of necessitation in his account. Take the case of a trope that acts as the basis for a causal power in an object. If that trope is in a circumstance appropriate for activation of its causal power, and given no intervening circumstances that would prevent the enacting of this power, will it be the case that the causal power manifests? If there is no necessitation, it is not clear why we ought to think that the causal power will manifest: without necessitation, it is consistent for the circumstances and the causal powers to be present with no intervening circumstances and yet the effect does not manifest. Campbell might object here that it could be otherwise: the circumstances that the basis finds itself in could have been different. But again, we have ontologies such as Lewis,' where even if the objects and their configurations had exactly resembled between instances, the results of their interactions could be different. Campbell's account does not allow for this. So he is at least letting in something enough akin to necessitation that other Humeans ought to deny his basis for causation, whether he wishes to call it necessitation or not. There is a further problem to be addressed in precisely how it is that tropes act as causes and what it is about a trope that allows it to serve as a causal basis. Campbell does not answer these questions with any level of precision, taking the only real answer to be based in scientific inquiry. To see how such an answer plays out, consider the case of a soporific again. That a soporific chemical causes drowsiness is based in the interactions of the chemical with chemicals already present in an animal. These interactions are what ground the soporific property, for

82 Ibid:123. 
Campbell. Generalizing from this, science can tell us how the underlying interactions cause the disposition to manifest. But he does not say in detail what it is about a trope that allows it to serve as a causal basis in this manner. While I think such an answer is possible, I will not speculate as to how Campbell might solve this problem, so that we might examine how he has done with respect to other desiderata.

\subsubsection{Field Theory of Tropes}

On our fourth desideratum, it seems that Campbell does better than any account thus examined in giving a true one-category ontology. However, this is upset in Campbell's account of change and gradation. As Campbell notes, many qualities such as hue and temperature either change by degrees or have fuzzy boundaries: the boundary between purple and violet in a rainbow being an example he uses. ${ }^{83}$ To deal with this problem, as well as problems with the boundary conditions of spatio-temporal parts, Campbell commits to what he calls a 'field theory.' This is an extremely sparse theory of tropes in which there are only a few actual tropes. These tropes are fields that exist across space-time without true spatio-temporal parts, and are things like the gravitational force, the strong nuclear force, etc. Particles, for Campbell, are merely, "A zone in which several fields all sharply increase their intensity." 84 The existence of particles and their features, such as spin and momentum, are then only accepted instrumentally on his theory. This is true for ordinary objects as well, such as tables, chairs, and persons. So, on this theory ordinary objects do not actually possess tropes but something that arises out of the true tropes of the world:

\footnotetext{
83 Ibid: 154.

84 Ibid: 148.
} 
fields. Campbell claims that the properties we ordinarily take to exist only exist as 'quasitropes.' He denies that these things are unreal: there are real variations in the field-tropes that account for the existence of these quasi-tropes. But he says the boundaries of the quasi-trope are is partially mind-dependent, as we create arbitrary but not random boundaries between objects with our judgment. ${ }^{85}$ This is enough for Campbell to claim that quasi-tropes are not genuine objects of ontology.

Notably, this turns Campbell's theory into a sort of monism. As objects only exist as a compresence of tropes, and as the only tropes are fields with variations across the entirety of space-time, he is committed to there being only one object: the universe as a whole. The fields are the tropes of the bundle, and since they all extend across all of space-time, the entire universe is a single object: a bundle of compresent tropes. Campbell does allow divisions of the field, but only as 'pseudo-objects' and 'pseudo-tropes,' not as genuine entities of his theory. ${ }^{86}$ The merits and demerits of monism have been greatly debated in philosophy ${ }^{87}$ and are beyond the scope of this investigation, but we may add it to the conclusions of and philosophical commitments of this theory.

This creates problems for Campbell with respect to our third desideratum. If Campbell is right, most of the properties and objects we take to exist do not actually exist aside from being 'well-founded appearances.' And yet, science has laws regarding particle spin, chemical bonds, and heat: all properties that Campbell denies as being anything more than well-founded appearances. If laws require some form of truthmaker to be true laws, then these laws cannot be well-founded, even if the appearance of the objects is. There is

\footnotetext{
85 Ibid: 152.

86 Ibid: 151.

${ }^{87}$ In recent years, most notably by Schaffer (2001) and his opponents.
} 
nothing ontological in the world, for Campbell, that matches up to these 'quasi-tropes.' And so there cannot be ontologically-grounded laws regarding them. Campbell admits as much in declaring the value of admitting such objects to be instrumental in nature. ${ }^{88}$ This consequence will require us to abandon ontologically grounding all laws in fields such as biology, chemistry, psychology, and economics, as well as most (though not all ${ }^{89}$ ) laws in physics.

In addition, I do not believe this strategy solves the problem that Campbell means for it to solve. Even if the whole of hadronic force across space-time is a single property, as Campbell claims, we still have nothing to account for variation and similarity within this field. Why does the field vary as it does? Even if particles exist only as compresent spikes in multiple amplitudes, why should they spike compresently? This is not a question for the physicist: if there are no objects or properties in the sense we normally think of them but only these fields, there is no Humean reason why they cannot vary except in compresent amplitudes. Just as Campbell notes that any ontology needs to admit that in the actual world there is sameness, any ontology needs to admit in the actual world that there are particles. Without some explanation of why the fields vary as they do, it becomes happy coincidence that the universe is comprised of a few standard kinds of particle that vary in predictable ways. This problem is actually worse for Campbell, as he rejects any underlying mechanism for his field theory and says that the true laws of nature only exist insofar as:

\footnotetext{
${ }^{88}$ Campbell, Properties: 149.

${ }^{89}$ Laws regarding fields would be notably exempt.
} 
We sum up the patterns of these (field) interactions in basic laws of nature, which are generalizations... other more superficial generalizations may derive from these basic ways of working. Or they may be mere coincidences. ${ }^{90}$

While a common critique of Humean laws of nature lies in regularity being nothing more than happy accident, for Campbell this problem surfaces with all ordinary objects, whose existence is also a happy accident. Campbell does nothing to ameliorate this, embracing it wholeheartedly. One might defend Campbell here, taking a universe without objects to be possible and as such, it truly being a mere happy accident that there are objects at all. But this is not the same objection as I am raising here. If objects as we know them exist only due to variations in fields and those fields interacting as they do cannot be explained by some law or set of laws that holds between fields as anything more than a generalization, then the fact that objects exist with any kind of stability in our world can only be a happy accident: it is entirely possible for the fields to change at any moment, creating or destroying any or all objects by virtue of the underlying phenomenon changing. While the possibility of an empty universe carries with it the possibility that our universe being one of objects is accidental, this is different than an ontology allowing that at each moment within our universe the existence of objects is accidental.

As a final objection, this field theory only works if we assume that the level of fields is the most basic, fundamental level. That is, that the fields themselves do not depend on some further substrata as ordinary objects depend on the fields of Campbell's ontology. This leaves Campbell's account open to destruction by any future science that calls into question the basic nature of his fields. Campbell may claim that the fields are ontologically

90 Campbell, Properties: 149. 
basic and nothing could act as their substratum. But I find it unlikely that scientists doing the work to understand why the quantum level operates in the manner that it does will be terribly persuaded on his mere say-so, and many are already theorizing about what might be occurring 'beneath' the quantum level. This is a problem because if the fields he relies on are themselves dependent on some other properties or objects, his solution to problems of change and gradation may no longer work at the more fundamental level.

A more modest objection would be that fields may yet depend on other fields, making Campbell's account accurate save for having misidentified the exact nature of the fundamental tropes. On this account, the fields currently known to physics of are really just more quasi-tropes, and the real tropes are fields of some more fundamental level. However, this line of reasoning is highly speculative. If fields depend on something else for their existence, there is no prima facie reason to expect that those things will then also be fields. In fact, Campbell's theory that ordinary objects rely on something as different as fields gives us reason to doubt this picture, as it allows for quasi-tropes to be wildly different than the tropes on which they depend. Many properties of ordinary objects are quite dissimilar from fields, and so if fields are yet another sort of quasi-trope, that the tropes on which they depend are fields requires investigation and arguments that have not at this point been provided.

One looking to rescue Campbell's theory from this objection might attempt to admit quasi-tropes as genuine ontological entities of the theory. But Campbell makes it clear that these are different in kind than tropes, although he does not recognize them as genuine entities. This would require reworking his theory of quasi-tropes. But even after this work it would only serve to make his ontology a two-category ontology: one of fields and quasi- 
tropes. That would render his theory no better than many realist theories or other twocategory ontologies in this regard. And even without this, the field theory he presents us with creates significant philosophical baggage. Namely: abandoning the ontological basis for all but the most basic physical laws, and only those respecting fields; accepting monism; accepting the hypothesis that there can be nothing more fundamental than these fields; and accepting the controversial claim that these fields are all that exist and do so independently of the particles which we normally ascribe them to. Further, there are open questions about whether or not his Humeanism about laws can balance with his other claims regarding them and problems both with accepting resemblance as a primitive of his theory and the analyticity of object properties even if we ignore the elements that his field theory brings in. While I think that Campbell's theory thus has several advantages, there are numerous problems that I believe his theory has introduced that need to be corrected for it to be a viable trope theory.

\subsubsection{Martin's Substratum Theory}

While Campbell's bundle theory may have its problems, it is not the only game in town for trope theories. Opposed to bundle theories we have substrata theories, which admit of properties but, rather than have them bundled in a relation of compresence, posit some underlying substratum in which the properties inhere. While there are historical examples of bundle theories such as the theory offered by Locke, ${ }^{91}$ there are few modern examples of substratum theory. One of these which I will examine is offered by C.B. Martin.

\footnotetext{
${ }^{91}$ John Locke, Essays Concerning Human Understanding, (London, Everyman, 1961).
} 
Before delving into the specifics of Martin's theory, I will examine one reason for substratum trope theory's lack of popularity in an attempt to give the substratum theorist their best due. This is that in terms of simplicity, it fares no better than two-category ontologies such as Armstrong's. Given the work one can accomplish with universals, it might seem silly to admit a two-category ontology but not admit universals into it. However, one might find universals suspect for other reasons, such as their multipresent or perhaps transcendent nature. If one is disposed to be skeptical of universals for these reasons and not reasons of parsimony then one might be sympathetic to a substratum theory despite its lack of advantage in comparative metaphysical economy.

\subsubsection{Attacks on the Bundle Theory}

Martin begins his defense of a substratum trope theory with an attack on the bundle theory. ${ }^{92}$ Martin attacks the idea that a bundle of properties can replace objects in one's ontology. Martin brings to this attack the fact that properties are always properties of something. A property cannot exist on its own, free-floating in the world: it must belong to an object. Martin compares a bundle of properties to a collection of objects. He points out that the collection is dependent on the individual objects that make up the collection: it cannot exist without them. He claims this is not so for properties. He does not deny that each object must have some properties, but that the object does not depend on its properties for its existence the way the collection depends on its individuals. Martin claims that the chain of dependency goes the other way: properties require their objects in order to exist.

${ }^{92}$ C.B. Martin, "Substance Substantiated," Australaisan Journal of Philosophy 58, No. 1. (1980): p. 7. 
A bundle theorist may, of course, deny this line of reasoning and give some argument that the chain of dependency doesn't go that way. But it is worth noting that it mirrors other problems for the bundle theorist. Bundle theories have difficulties with property change in a given object, ${ }^{93}$ in large part because if an object is merely a colocation of tropes, then eliminating a trope from the bundle or adding a new one makes it a different bundle. Elsewhere, Martin puts this problem even more strongly:

One must avoid a use of the term "identity" such that any entity over time can be said to lack continuing identity simply because it has changed properties or has lost some parts or added or had substituted some parts. ${ }^{94}$

This is also reflected in our earlier criticism of Campbell, which noted that his bundle theory rendered an object's having its properties necessarily. ${ }^{95}$ While not the same criticism as Martin's, both share a common thread: if an object is a bundle of tropes, the tropes cannot change and yet the object remain the same. This not only runs contrary to our intuitions, it runs into problems with our third desideratum. Scientific laws often feature change in some property of an object in their description. If objects do not truly change, at minimum a bundle theorist must explain how we can ground scientific laws that feature object change if they are to satisfy this desideratum.

Martin considers an alternative to substrata: linking properties to a spatiotemporal location for individuation. ${ }^{96}$ Ultimately, he finds this wanting for reasons similar to his problems with the bundle. If properties are not of an object but of a spatiotemporal location, then any motion in the bundle of properties will cause it to be a different object. In

\footnotetext{
${ }^{93}$ Simons (1994) notes this problem.

94 C.B. Martin, “On the Need for Properties" Synthese 112, No. 2. (1997): 198.

${ }^{95}$ As noted earlier (p. 74-6)

${ }^{96}$ Martin, "Substance Substantiated,": 8.
} 
fact, the mere passage of time will reconstrue what was a single object as innumerable objects existing at different times. He ultimately does not reject that view entirely: he admits that one can accept an ontology with objects such as these. But he notes that there is theoretical work to do both for the philosopher and for the scientist should this theory be accepted and that it is strongly anti-intuitive.

\subsubsection{Substrata}

So what is a substratum? Martin notes that a common criticism of substratum theories is that the substratum seems to be a mysterious, 'I-know-not-what.' ${ }^{97}$ To make substrata less mysterious, Martin defines substrata as, "that about an object that is the bearer of properties." This he says is what a substratum is qua substratum. But this raises the question of whether or not this is itself a property. If it is, then how does the substratum bear THIS property. If it is not a property, then how are we to understand substrata?

If a substratum is really just "that about an object that is the bearer of properties," then this cannot itself be a property for Martin. Martin says that all genuine properties are tropes. Thus, while this elocution of what it is to be a substratum is similar to how we may talk about a property and is a genuine entity of his ontology, it cannot be a trope. If it is, then Martin is back to having a bundle theory with one theoretical addition: the property of being a collection of properties. ${ }^{98}$ But this would render objects 'mere collections' as he would have it and would thus fall prey to his own criticisms of bundle theories. An alternative would be for the substratum to be a relation between the properties. While

\footnotetext{
${ }^{97}$ Martin, "Substance Substantiated,": 4.

${ }^{98}$ Not all bundle theories accept the existence of such a property, but if that which about an object is the bearer of properties is a trope, then the theory would be a bundle theory which accepts such a trope.
} 
Martin clearly commits himself to the existence of both properties and relations, ${ }^{99}$ he does not explicitly commit himself to relations being tropes, which opens the door for this strategy. ${ }^{100}$ There are a number of ways this could go for Martin, depending on the nature of the relation, but as I think Martin must reject this idea in all of its forms, I will not examine them here.

I think there are three primary reasons Martin must reject substrata as relations. First is his definition of substrata. If the substratum is a relation and also 'that about an object that is the bearer of properties', then what are the relata of the relation? Clearly the properties would be one side of the relation, but what about the other? It cannot be the object, as Martin claims the object is just the sum of its substrata and its properties. ${ }^{101}$ This would result in the definition of object being circular. And it cannot be anything else, as there is nothing to the object but its substratum and its properties. But even overcoming this, Martin cannot commit himself to substrata being a species of relation. For if it is then substrata will fall victim to his criticism of the bundle theory. Just as a collection is dependent on its members for its existence, so too is a relation dependent on its relata. If substrata are a kind of relation, then he is as vulnerable to his criticism of the bundle theory as Campbell is. If the substratum is a relation between the tropes then as an object gains and loses property what the relation relates changes. As the substratum then is merely the relating of those properties which it relates, changing those properties gives us a different relation, altering the identity of the object just as it does for the bundle theorist. Lastly, in

\footnotetext{
${ }^{99}$ In "On the Need for Properties," (p. 196) and "The Need for Ontology," (p. 514), among others.

100 Martin also does not commit to relations not being tropes, so if substrata are not a species of relation, as I will argue they cannot be for his view, then this opens the door for relations to be tropes. Should this not work, Martin may need to commit to a three-category ontology.

101 Martin, "Substance Substantiated,": 7.
} 
his replies to Martin, Armstrong notes that Martin takes relations to be a kind of trope. 102 Martin, in his reply to Armstrong's reply, does not mention this interpretation despite responding to many other criticisms Armstrong levied against him, including many of at least as technical a nature and as important to his theory. ${ }^{103}$ So we can conclude that at very least, Martin takes relations to be tropes and so substrata are not a form of relation in his eyes.

If substrata are neither properties nor relations, then how are they features of objects? Here Martin may simply have to deny that a 'feature' of an object is the same as a property. Martin denies linguisticism about properties ${ }^{104}$ and so he may deny something similar here. Our language may lack a way to describe something about an object that is not a property and so it may be difficult or impossible for him to describe an aspect of an object that is not a property without using the language of properties, but this in and of itself is not a mark against his theory. It is at most a feature of our language. But the problem still arises in this form: how can the substratum do its work of bearing properties without itself being a property or a relation? At minimum, this would require distinguishing what it is for something to be a property in a manner that excludes substrata in a principled manner, and Martin does not do this.

102 C.B. Martin, David Armstrong, and U.T. Place, ed. Tim Crane. Dispositions: A Debate, (Routledge, New York, 1996): 98. 103 Ibid.: 126-146.

104 Martin, “On the Need for Properties,": 193. 


\subsubsection{Resemblance for Martin}

Setting this problem aside, we at least have an answer to our first desideratum: what it is to be an object is just for it to be a substratum plus some number of tropes, and for an object to have a property is for some trope to be borne by a substratum. So what of our second desideratum? Here I would like to call attention to two passages in "Properties and Dispositions:"

This requires a mind-shift from the philosophers' usual emphasis upon exact or inexact similarity between objects (that need a respect in which similar) to exact or inexact similarity between a specific property or respect (of an object) that since already detected or specified needs no further respect in which the respects or properties are similar. (p. 72)

And later:

... it is the resemblance or difference between the properties of objects, events or states of affairs that is basic to the resemblance or difference between objects, events, or states of affairs. Two objects are similar to and different from one another in virtue of the similarity to and difference from different things about (properties of) the objects. (p. 93)

This, I believe, gives us Martin's answer. Like Campbell, Martin takes resemblance of properties to be a primitive. The only way to analyze similarity or difference, for Martin, is in terms of the similarities and differences between properties. I take this answer to have the same problems for Martin as I noted for Campbell, and so will not make mention of those same problems again. However, Martin may be unperturbed by this. Martin draws special attention to our ability to discern that some properties are similar and others different in his presentation 105 and we might take him to mean that the only analysis of similarity and difference is to be in terms of the actual distinctions we make in our

105 C.B. Martin, "Properties and Dispositions," in Tim Crane ed. Dispositions (Routledge, New York, 1996): 72. 
examinations of properties. Take for instance 2 mass properties. We can compare these properties: one may be of a greater magnitude than the other, or we might note that both are responsible for a gravitational force. On this reading, Martin argues that comparisons such as these are the only form of analysis that we can present regarding the similarities and differences between these properties. If this is so, the problem may be weaker for Martin than Campbell, who takes this to be a primarily philosophical problem. ${ }^{106}$ I find persuasive the argument that our second desideratum is primarily a philosophical problem distinct from the question of how particular properties are similar or different, and so I think the problem of resemblance as a primitive is still a problem for Martin. But I recognize that Martin himself may not agree.

Martin may also have the resources to avoid my criticism of Campbell entirely. While Campbell explicitly says that resemblance is unanalyzable, Martin does not make this move. It is true that Martin claims resemblance as a basic element of his theory. But it is an open question whether or not resemblance is taken as basic qua a theory of properties, or if it is taken as basic simpliciter. If the latter, Martin's theory is vulnerable to the same criticisms of unanalyzable primitives used against Campbell. If the former, then it is not subject to the same criticism. This may be the better route for Martin, as he does not take the problem of universals to be primarily what his theory is about explaining. Martin need not give an account of what it is for two objects to resemble for this to work: he need only leave the door open for theorizing. Yet if he takes this route, his theory is still weaker than one which includes an analysis of similarity, given that one accepts that it is part of the work of an account of properties to explain similarity.

106 Campbell, Properties: 28. 


\subsubsection{Grounding Laws in Dispositions}

With respect to our third desideratum, Martin grounds the truthmakers of scientific statements in dispositions. This invites the question of whether dispositions are a species of trope. Martin says they are not. Instead, he claims that all properties are dispositional: that they have a dispositional as well as categorical character. ${ }^{107}$ This dispositional character of tropes may serve as the ontological basis for laws of nature, giving some answer to our third desideratum. But this alone may not resolve the issue, as Martin also notes that properties can be the bearers of other properties. ${ }^{108}$ So one might think that dispositions are a sort of trope for him, merely a trope that is borne by other tropes. These secondary tropes would be dispositional, leaving the properties that bear them as categorical properties. But not only does Martin explicitly deny this, accepting it would create real problems for his theory. ${ }^{109}$ If all properties are dispositional, then the dispositional properties would need some further dispositional property that could be ascribed to them. This invites a regress with respect to what dispositional properties a dispositional property itself bears. But Martin does not take this route, instead claiming that it is a part of being a property that it has two distinct, ineliminable characters: one dispositional, one categorical. One may object that a single trope may only have a single character: that if there are multiple characterizations there ought to be multiple tropes. But many common properties do not have a single dispositional character, instead having

\footnotetext{
107 Martin, "Properties and Dispositions,": 74., "On the Need for Properties,": 202. (among others)

108 Martin, "Substance Substantiated,": 4.

${ }^{109}$ Martin explicitly points out that this is a difference between his theory of dispositions and that of U.T. Place in Dispositions: A Debate. (p. 80)
} 
many. As a mass increases, its gravitational pull increases but so does the object's inertia. If a trope can have multiple, different dispositional characters, it's having a categorical nature as well is no more egregious. ${ }^{110}$ It is this dispositional character of properties in which he grounds the truth of conditionals, including scientific laws. ${ }^{111}$ As such, a scientific law is true if it accurately describes a genuine disposition of properties in the world. Here I agree with Martin, and a fuller defense of this way of grounding the truth of scientific laws will be presented in Chapter 4.

But there is an issue of metaphysical economy to be raised here. Already, it is notable that Martin's view is a two-category ontology: one of tropes and substrata. But we might further worry that he has added a third item to his ontology: dispositions. I do not think this is a genuine worry but it bears mentioning. The reason I do not think this is a worry for Martin is that dispositions are not an independent theoretical entity that is assumed for the success of the theory. Instead, Martin is describing an aspect of what it is to be a trope. In other words, saying that tropes have a dispositional character is different than saying that there is a separate, independent entity (a disposition) to which we must also commit which belongs to the trope. Rather, he is presenting us with what it is to be a trope. As such, there is no real issue of metaphysical economy here, at least not with respect to the fourth desideratum defended in Chapter 1.

\footnotetext{
110 One may object here that this is precisely why dispositions ought be distinct from the tropes that bear them. The argument becomes more complicated than I can devote time to here, but as it is relevant to the account given in Chapter 4, I will address some of this argument there.

${ }^{111}$ C.B. Martin, “Dispositions and Conditionals," Philosophical Quarterly 94, No. 174 (1994): p. 2.
} 


\subsubsection{Final Considerations for Martin}

Martin's view does fall victim to the fourth desideratum, at least over singlecategory ontologies such as the bundle theory. Further, even if the bundle theory is not a workable single-category ontology and even if there are no workable single-category ontologies, the fourth desideratum still creates a tension between Martin's account and realist accounts. If both perform the same work and with the same metaphysical economy then other criteria will be needed to decide between them, if there is a rational way to decide between them at all. And there may be reasons to favor a realist account over Martin's view. For one, while Martin favors tropes for his theory he does not truly reject universals and may be willing to modify his theory to include them. ${ }^{112}$ And while both states of affairs and substrata are primitives of their respective theories, Armstrong maintains that we have experience of states of affairs while Martin admits that we can have no direct experience of substrata. This experiential element adds credence to Armstrong's theory over Martin's theory with universals rather than tropes. If Martin's theory is to be a match for a realist such as Armstrong, it will either be because one favors tropes over universals or due to an assessment of the metaphysical baggage associated with the account.

With respect to limiting unanalyzed philosophical baggage, Martin does neither especially well nor especially poorly. Resemblance is taken as an unanalyzed primitive of Martin's theory, but for reasons outlined earlier this may be less of a problem for Martin than it was for Campbell. Dispositions are similarly basic, but one can hardly call them unanalyzed: Martin spends a good deal of time outlining what it is for something to have a

112 Martin, "Properties and Dispositions.": 72-3 
disposition and how dispositions relate to tropes. Substrata are taken as basic, and while he provides some account of what it means to be a substratum qua substratum, we may find this account inadequate for reasons explicated earlier. There is also the issue of what it is to be a property. But here Martin gives us some analysis of what it is to be a property: a property is some ontological characteristic of an object both categorical and dispositional in character. This would seem to rule out abstracta, such as mathematical objects, unless they can somehow be shown to be objects.

A final worry for Martin may be presented in that he commits himself to neither a sparse nor an abundant theory of tropes. Like others in the debate, Martin uses many properties in his examples that would suggest a more abundant theory of properties. But this does not guarantee a commitment to them: many theorists use properties for the purpose of illustration that they later abandon as being non-genuine. However, some work of his suggests a theory of properties that includes more than the base properties of physics, at minimum. In his work "Identity and Exact Similarity," Martin uses his theory of properties and dispositions to analyze cases of personal identity for human individuals. If one does not admit human individuals into one's ontology, favoring a more barren metaphysical landscape, then this sort of analysis does not hold water. To give this argument its due, he at least includes human individuals and some basic physical properties in his ontology. But while his ontology would rule out some theoretical constructs such as properties without dispositions, it does not rule out entities such as numbers, absences, disjunctive properties, or other potential properties that some philosophers have found suspect. Accordingly, while we can affirm that his ontology is not sparse in the most barren sense of the term, we cannot confirm if there is an upper limit to 
the properties he can and will accept in his ontology. Many of these properties are demonstrably problematic and if Martin allows for them then this creates problems for him. Yet this may be a minor issue at best, as I see no clear reason why he must admit such problematic properties. His theory might do better for explicitly putting boundary conditions on what properties he allows, but I do not know of any reason to suspect that he must allow properties that are philosophically suspect. ${ }^{113}$

\subsubsection{Conclusions for Theories of Properties}

While both realist and nominalist theories can give responses to our first two desiderata they do so at significant cost to the fourth and fifth, albeit in different ways. Realism implicitly accepts at least two kinds of fundamental entity while nominalism makes its own tradeoffs in attempting to answer the problem of universals. With respect to the third desideratum each has its own problems. For realists assuming the existence of laws of nature as entities the question arises whether or not these laws of nature are governing or non-governing. If they are governing then by what means do they tell objects how to behave? If they are non-governing then in what sense do they participate in the particulars or states of affairs that we take them to participate in? For nominalists, addressing the third desideratum seems to either invite psychologism or force us to abandon an ontological grounding of laws of nature. It may be possible to save realism or nominalism from these problems but it is not clear it can be done without taking on even more philosophical baggage or reducing the simplicity of the accounts given.

\footnotetext{
113 This assumes that one does not find mental properties to be suspect, as he does explicitly defend these. However, if pressed I think he could even eliminate these from his ontology without concern for the rest of his work and so even this is a minor issue at best.
} 
Trope theory might then prove the high road. While theories such as Campbell's and Martin's may be problematic in their own right, each illustrates benefits of a trope theory and reasons to favor one. Campbell shows that a true one-category ontology is possible, albeit with difficulties. Given our fourth desideratum there are significant reasons to investigate if a one-category ontology is viable, as prima facie there are reasons to favor a one-category ontology over alternatives. Martin's lack of a one-category ontology shows why it is important for a trope theorist to develop one, as without it there is no clear reason to favor the trope theory over the many realist two-category ontologies that exist. At minimum, such a defense is going to be more difficult and something extra will be needed for a theory to emerge as a decisive victor..$^{114}$

But even if a bundle theory of tropes can give us a one-category ontology and thus has a prima facie advantage over other theories of object properties, Campbell's defense of such a theory and Martin's defense of substratum theory both illustrate problems that such a theory should solve. One must account for the analyticity of particular objects having their properties, either by biting the bullet or making some distinction that dispels the problem. Similarly, one must account for at least the appearance that properties depend on their objects, as a bundle theory normally puts this dependency in the other direction. While both Campbell and Martin take resemblance to be a primitive, providing some analysis of resemblance will make such an account stronger, as not doing so ran into problems with our second and third desiderata. There is real pressure to give such an

\footnotetext{
114 As I will demonstrate in Chapter 5, having something that fills this role is good for any theory. I will attempt to provide such a benefit for the theory defended in Chapters 3 and 4: that of empirical falsifiability.
} 
analysis as both realist and nominalist theories can do this without simply taking resemblance or similarity to be a primitive of their theory.

With respect to the third desideratum, dispositions can provide a solid basis for a trope ontology's grounding of the truth of scientific law statements. The trope theorist does need to account for whether dispositions are themselves tropes or, as Martin would have it, aspects of tropes. However, there may be other paths open for the trope theorist and some examination of the benefits of turning to dispositional accounts will be necessary, as we will see in Chapter 5. The final desideratum is the most complicated: there is no clear, single list of questions that allow a theory to escape metaphysical baggage. Even an honest attempt to address the problems that have arisen for other philosophers may yet result in unasked questions later proving problematic for a theory. But by knowing some of the pitfalls of other theories, both trope theories and others, we may create a theory that at least has some of these questions in mind from its inception. This is a strong advantage for the construction of a new theory. 


\section{Chapter 3: Constitutive Trope Theory}

Given our fourth desideratum of assuming the minimum number of entities for an account and theories such as Campbell's showing that a trope theory can provide an ontology with only one kind of object, there is a significant prima facie advantage to trope theories over realist theories. And given the problems demonstrated for a nominalist providing an ontological grounding for laws of nature, the trope theory has an advantage over the nominalist with respect to this, our third desideratum. These are, of course, not fully decisive. It may be that accepting a trope ontology brings with it so many problems and unanswered questions that we nonetheless are forced to turn away from it. Further, as many trope theorists take similarity as a primitive that either is not or cannot be analyzed on their view, trope theories may be weaker with respect to our second desideratum of explaining similarity between particulars than alternatives. Nonetheless, given the considerations in favor of the trope theory, it is a good starting point for our investigation.

As we are attempting to start with a one category ontology, we will begin with the commitment to there being only one fundamental kind of entity: that of trope. By fundamental, I mean that any other entities admitted must merely be a sort of construction of the more fundamental entity. While in this account it will prove necessary to talk of objects, relations, and other kinds of entities, each entity in question must either be a sort of trope or be constituted entirely of tropes. For instance, relations will be a kind of trope on this theory.

There are at least three questions that this account must answer:

1) What it is for an object to have a property; 
2) The problem of universals, or how it is that two objects can have similar properties

3) To provide an explanation for laws of nature

Any answer to these questions will, naturally, result in more questions that will require answering. However, before we get started I will need to make a terminological distinction. It will be important as the account goes on to talk about genuine properties, that is, properties that are admitted of in the ontology, and non-genuine properties that can be reduced away or eliminated entirely. This distinction is required because some properties are philosophically suspect: there are a number of predicates and concepts that while useful, may not pick out something that exists in the world. For instance, I can refer to the mystical healing properties of a unicorn's horn without thinking that unicorns, their horns, or magic exists. In the case of properties like 'being magical' it may even be such that, once properly defined, it is impossible for them to exist. There are also cases involving predicates where it is not entirely clear which properties they are meant to capture, such as baldness. Exactly how many hairs, or what sort of coverage, is necessary in order for a person to have the property of baldness? Clearly a person with no hair is bald. And even if a person has 1 hair on their head, we would still say they are bald. And a person with a full head of hair is not bald, as is that same person after a single hair is plucked from their head. But where the boundary is between being bald and not is unclear. For properties with fuzzy boundaries such as this, many philosophers respond by saying they are not genuine properties: one might have a certain number of hairs on their head, but there is no property of baldness that accurately captures our use of the term.

But at times we will need to refer to genuine properties, non-genuine properties, or both together. For sake of clarity, when I use the term 'property,' I am referring both to 
genuine properties and non-genuine properties. When I use the term 'trope,' I am referring only to the genuine properties. A single term for non-genuine properties is harder as there are many ways for a property to be non-genuine, but I will try to point such properties out as time goes along.

\subsection{Tropes}

Two standard answers to the first question, as previously examined, are bundle theories and substratum theories. That is, either what it is for an object to be an object is merely for it to be a collection of compresent tropes, or that there is some 'bare particular' that is not itself a trope but which unites the tropes. Given our fourth desideratum, rejecting bare particulars is to the benefit of the theory. Yet there are problems for a bundle theory as well. Here I will be offering an alternative that, while technically a bundle theory has many of the benefits of a substratum theory. On this theory, objects will be bundles of tropes. However, these bundles will not be present at the locations they are because of a primitive compresence relation. Instead, these bundles will be present at the locations they

are because of lower-level objects upon which they depend. In this way, something akin to a substrate will be present to ground the existence of the bundle, but this will not be a bare particular and will, in fact, entirely consist of tropes.

\subsubsection{A Theory of Constitutive Parts}

To get a single-category ontology, we will posit a bundle theory of tropes. Once we have established that an object is nothing more than a compresence of tropes, we need to account for this compresence. Take the case of an apple, which may have properties of 
being red, having a particular mass, shape, flavor, etc. Assuming that these are all tropes and if the apple is nothing other than these tropes, it bears asking why it is that these tropes compose an apple, or why it is that this particular red-trope and this particular masstrope should be tied together in such a way as to form an apple. Biological science actually gives us something of an answer here. The apple has the properties it does because it is composed of certain cells and chemical compounds. That these cells and compounds exist, and their relation to the other cells that form the apple, are what lead to the apple having the properties that it does. I theorize that this sort of relation is what binds all tropes to their object. The trope exists only because certain relations between lower-level objects exist. The redness, mass, and shape of the apple are all compresent because the same lower-level objects are responsible for each of them. These lower-level objects are themselves compresent bundles of tropes, in the same manner as their higher-level object is. In this way, some of the benefits of a substratum theory are obtained without positing bare particulars. I will refer to these lower-level objects as parts.

It is important that the notion of part here is not that of a "mereological" part. A mereological part is an arbitrary region of spacetime that is within the spacetime boundaries of an object. This is not the notion of 'part' used here. Rather, it is more similar to the use of the term part to describe the parts of an engine or the parts of a table that one is assembling. If one is assembling a table, we intuitively think of the parts as being the legs, the top, any screws or fasteners that may be used, etc. We do not generally take the parts of a table to be arbitrary regions of spacetime. The leg of a table is its own object, but it is also a part of the table. This is the notion of part we are trying to capture. So each part is itself an 
object: that is, a bundle of compresent of tropes. ${ }^{115}$ I'll refer to this notion of part as "constitutive parts" when distinguishing the two, for sake of clarity.

Each constitutive part, rather than being defined by a region of spacetime, is instead defined as being an object: a bundle of compresent tropes. But not all bundles of compresent tropes that are spatially co-located with an object will be constitutive parts on this view. This is because an object may, at least in principle, be spatially-colocated with another object but have no bearing whatsoever on the properties of that object. Instead, any object must meet two considerations to be a constitutive part. First, the object must exist within the spacetime boundaries of the object to which it is a part. Second, the object must, through relations with other objects that also fulfill our first criterion, be responsible, in part, for the existence of tropes in the higher-level object.

So what is the relationship between constitutive parts and the tropes they are responsible for? What does this 'responsibility' amount to? Here I mean it to be a supervenience relation. That is, that there is nothing to the existence of the higher-level tropes except for relations between the parts that compose them. ${ }^{116}$ The tropes exist when and where they do because the relations between the constitutive objects exist where they do. Nonetheless, this is not a reductive analysis of tropes: it is not the case that we can eliminate the higher-level tropes and talk only about the relations between the lower-level parts. There are two reasons for this.

\footnotetext{
115 One might wonder why we need objects to act as parts. One might propose that tropes instead be the constituent ground for other tropes. While this sort of account might be possible to construct, in actual cases of composition it is often the case that multiple properties of the component object are what give rise to the higher-level property. Objects as parts more accurately reflect this relationship than tropes as parts. 116 This version of emergence is the same one used by Campbell (1996)
} 
The first is that certain scientific laws will require that we discuss properties of the higher-order level and giving these laws ontological status will require that we give their terms such status. For instance, Boyle's Law requires that we talk about the volume, pressure, and temperature of a gas, even though the gas reduces to molecules in certain spatial configurations. Even if the spatial configuration of the molecules of the gas changes, under Boyle's Law, provided that the temperature and pressure remain constant, the volume of the gas will as well. If we were to eliminate properties such as volume, pressure, and temperature in favor of talking about the spatial configurations of the molecules, we would not be able to grant Boyle's Law, or any other law concerning higher-level tropes, a place in our ontology. For a law to have an ontological grounding, rather than a merely pragmatic grounding, the properties which the law-statement purports to describe must exist ontologically.

In addition, certain properties will be able to be grounded in more than one set of relations between constitutive parts, in other words, may be multiply realizable. While problems of multiple realizability are common enough in discussions of philosophy of mind, we do not need to invoke the mental to have such overdetermination in our ontology. Take the case of a white cube put in a room with monochromatic red light such that the cube appears red. If we take that same cube and apply the appropriate shade of red paint to one half, the cube will still appear to be the same shade of red on the painted and unpainted portions. This shows that the relational property ${ }^{117}$ of "appearing red" in this case is overdetermined for the painted side of the cube. If we had not painted half of the cube it would

\footnotetext{
117 This property is complicated, but in ordinary circumstances involves an object, an observer, and light acting as an intermediary.
} 
have still appeared to be the same color given its conditions. It is also the case that this same section would appear red if it was not put in that particular lighting. ${ }^{118}$ So neither the paint nor the lighting is necessary for the cube's appearing red, but each is sufficient for it. We can contrast this with the unpainted portion, where the redness property is not overdetermined. In other words, if we switch the red lighting for white lighting, one half will retain the redness property while the other will lose it. If the redness reduces to a set of relations between the properties, there are significant difficulties in providing an ontological grounding for the disjunction of states of "being in monochromatic red lighting" and "being painted" in describing the cube's redness without admitting disjunctive properties. However, these worries can be avoided entirely if the redness of the cube is a distinct property from the relations between parts that are responsible for the redness even if the redness supervenes on those relations. This allows us to spell out the supervenience relation in more detail in that the relationship between constitutive parts and their tropes is a sufficiency relation. Formally, a collection of objects P are constitutive parts of an object X iff the relations between $\mathrm{P}$ are a minimally sufficient condition for one or more of the tropes that are constitutive of X. "Minimally" is added to the above, as it is trivially true that if relations between two objects, A and B, are sufficient for the existence of a trope $\mathrm{T}$, then the addition of the relations of $\mathrm{A}$ and $\mathrm{B}$ to some other object $\mathrm{C}$ will also be sufficient for $\mathrm{T}$, provided that $\mathrm{A}$ and $\mathrm{B}$ are in an appropriate configuration and their relationship to $\mathrm{C}$ does not render the existence of $\mathrm{T}$ impossible. ${ }^{119}$ Thus, making it a

\footnotetext{
${ }^{118}$ It should be noted that, on the final version of this theory, this will not be problematic. As each set of conditions sufficient for a property's existence ontologically grounds that property, the fact that a property is overdetermined is not especially problematic.

119 Of course, there are many cases where the condition that the relationship to $\mathrm{C}$ does render $\mathrm{T}$ impossible. These will be discussed in the discussion on antidotes in Chapter 4. The important point here is that when
} 
minimal sufficiency requirement eliminates certain vicious problems of overdetermination involving parts that are irrelevant to the existence of a given trope.

The sufficiency condition here is a kind of sui generis sufficiency condition that I call 'existential sufficiency.' That is, the mere existence of the constituent parts in the relations they are in is sufficient for the existence of the trope. This is distinct from causal sufficiency, as causal relations exist in temporal sequence while the being existentially sufficient is contemporaneous. If an event is causally sufficient for another event, the former will occur before the latter. But in the case of existential sufficiency, the trope in question exists precisely as long as the relations between the relevant constitutive parts exist and no longer. For $\mathrm{P}$ to be existentially sufficient for $\mathrm{Q}$ is for it to be the case that as long as $\mathrm{P}$ exists, Q exists, and that $Q$ cannot exist independently of $P$. For instance, I am composed of organs and only exist so long as some collection of organs exists in relations appropriate for maintaining my existence. Each of my organs is composed of cells, and those organs only exist as long as some collection of cells exist in relations appropriate for maintaining their existence. This is unlike causal sufficiency as throwing a brick through a window may be sufficient for that window's breaking but removing the brick from its relation to the window after the causal interaction does not alter the window's status as broken or undo the breaking, whereas removing some of my organs may lead to my existence ceasing.

A technical problem arises when we consider emergence, however, as it must be contrasted with identity. Take a given volume of gas with a particular temperature. AIs this temperature-property emergent from the relations between the constitutive parts of the

$\mathrm{A} \& \mathrm{~B}$ are jointly sufficient for $\mathrm{T}$, it is not the case that every $\mathrm{C}$ to which they have a relation is also a constitutive part of $\mathrm{T}$. This can, of course, be made more complicated if the conjunction of $A \& C$ is also jointly sufficient for $\mathrm{T}$. 
gas or is it identical with some feature of those parts? The kinetic theory of heat tells us that temperature simply is mean kinetic energy. If this is so, there is no emergent property of 'temperature' here, but rather that temperature is merely a convenient means to describe the average kinetic energy of the molecules of the gas.

This can be contrasted with the color absorption properties of an atom. An atom absorbs and reflects the wavelengths of light that it does because of the vibrational frequency of its electrons. If these electrons have the same vibrational frequency as a given wave of light, that wave will be absorbed and transformed into vibrational motion. So is the color absorption identical to the vibrational frequency of the electrons, or is it an emergent property of the atom with the electrons being the relevant constituent parts? The answer requires us to examine why electrons in atoms absorb different vibrational frequencies: that is, why do they absorb the wavelengths of light that they do. The answer is that while all electrons have some vibrational frequency, that frequency alters based on the electron's orbital if it is a part of an atom. So it is the relationship between the electron and the nucleus of the atom that grants the electrons their particular vibrational frequency. The frequency of light that the electron absorbs is identical to its vibrational frequency, and this frequency is an emergent property of the atom based on the electron's orbital. Thus, 'color absorption' as a property is identical to vibrational frequency (in cases where the frequency matches that of some wavelength of light) and such frequencies are an emergent property as it is not merely a property of some parts of the atom but is instead grounded in the relations between those parts.

The above is the characterization of the relation between constitutive parts and the tropes that they consist of, but more can be said. While each constitutive part is itself a 
bundle of tropes, not every property of the constitutive part will be relevant to a given supervening property that is present in the object to which it belongs. Take the example of a white wall. On this analysis, the whiteness of the wall will supervene on the relations between the molecules that form the wall. But it is notable that it is the "color reflection" trope of the molecules that is relevant to the wall's being white. The molecules have other properties, such as mass, which may have no relevance to the whiteness of the wall. Similarly, the mass of the wall supervenes on the mass of the particles that make up the wall but is at most contingently related to the color absorption of those same molecules. Solving this technical detail will require us to distinguish between two sorts of supervening properties: aggregates and emergent tropes.

\subsubsection{Aggregates and Emergent Tropes}

Aggregates are not true tropes. An aggregate is a sort of pseudo-property that exists only in virtue of being a collection of some properties of the constitutive parts of the object. These pseudo-properties can be reduced to the summing of the properties of the constitutive parts of the object with no ontological loss. Further, there are both Occamist reasons to eliminate aggregates from our ontology, as well as other worries. Take the case of a white wall of a single hue and shade. Each of the molecules that make up the outer layer of that wall will absorb certain wavelengths of light, contributing to its whiteness. But the color of the wall is not a different property than the light absorbing properties of the molecules that make up its outer layer. Instead, the color of the wall is identical to the collection of relevant properties on its outer layer. This is an example of an aggregate. In an emergent trope, the lower-level objects that are responsible for the existence of the trope 
are sufficient for its existence, but in the case of the aggregate the lower-level properties, taken in total, are identical to the higher-level property. This distinguishes aggregates from emergent tropes. There is another type of aggregate that we need to consider, as best shown by the property 'velocity.' An object's velocity is nothing more than its speed and the direction it is travelling in. Thus, velocity is another aggregate, as it can be harmlessly eliminated from our trope ontology by reducing it to an aggregate of the object's speed and the direction of its travel. Most aggregates reduce to some set of tropes in this way and as such, the aggregate is not truly eliminated but is instead reduced to the tropes that constitute it. However, we can find an exception in the case of numerical properties. Take 5 apples on a table and consider the pseudo-property of "five-ness." This is not only an aggregate, it is nothing but an aggregate: it reduces to the fact that there are five apples, but there is no lower-level numerical property for the apples as there were lower-level physical properties in our earlier examples. Numerical aggregates reduce not to a lower-level set of like-properties, but to the mere existence of the objects on which they supervene. The "fiveness" reduces only to the fact that there are five objects in the aggregate, not to any numerical "one-ness" property that each bears. In this way, numerical properties are not only eliminated in specific instances as was done with mass aggregates but are instead eliminated from our ontology as a kind.

The ability to eliminate aggregates from our ontology harmlessly shows one fact about tropes. Each trope is taken to be an atomic property, in the sense that it cannot be divided into sub-properties without losing some important fact about the trope. This has a number of side-effects, especially when we consider potential tropes such as length and 
duration, where the property in question might be able to be subdivided indefinitely. This will be discussed more when we talk about such tropes.

As we have eliminated aggregates from our ontology, the only non-foundational tropes remaining will be those that arise through emergence. An emergent trope is one whose character is supervenient on the relations between its constitutive objects, but where the emergent property is non-identical to the collection of lower-level properties. Consider, for instance, the surface tension of a molecule of water. While this surface tension is due to the structure of the hydrogen and oxygen particles that make up the molecule of water, neither the hydrogen nor oxygen atoms themselves can be described as having a surface tension per se. Nor is it the case that the surface tension is merely some other collection of properties of the molecule of water: it is a new property that arises out of the parts of the water molecule and their relations to one another. As the character of the surface tension of the water cannot be wholly described as either an aggregate of some property of the parts of the molecule of water nor as a collection of some other properties of the molecule of water, it is an example of an emergent trope.

The difference between aggregates and emergent tropes can be further illustrated if we consider the light-reflecting properties of the white wall in our earlier example. The 'whiteness' of the wall is not, on this ontology, a true property. It amounts to nothing over and above the color reflecting properties of the molecules that make up the outermost edge of the wall. The color reflecting properties of those molecules, however, do not merely amount to some color reflecting property of still lower-level parts as the lower-level parts individually do not possess such a property. Thus, the whiteness of the wall is a mere 
aggregate of the color absorbing properties of each molecule of the outer surface of the wall which are themselves emergent properties.

While the tropes we are familiar with ${ }^{120}$ are emergent, there is another sort of entity that we need to consider: foundational tropes. This theory will have very little to say about such tropes for the time being and foundational tropes will prove to be a unique challenge for this theory. For now, talk of these tropes will be bracketed while the rest of the theory is constructed, as most if not all examples of tropes which mankind is presently aware of are emergent tropes.

\subsubsection{Eliminable Properties}

Having discussed the how aggregate properties are not genuine properties for constitutive trope theory, I would like to now turn our attention to three cases where we can eliminate the properties from our ontology entirely: those of negative properties, and historical properties. By showing how we can eliminate these we not only keep at bay certain potential problems that may arise from them, but also show some of how one might use this theory to simplify one's ontology. We will start with the case of negative properties. A negative property is defined in terms of a lack of existence. For instance, an object may lack a charge, or lack some other property. I think a common first-instinct is to eliminate these from one's ontology and while I think this is correct, more needs to be said if we are going to have a theory of tropes that can account for laws of nature, as many laws of nature

\footnotetext{
${ }^{120}$ I do not believe that, as of this writing, humanity has discovered any foundational tropes. More will be said on this later.
} 
include what amounts to a ceteris paribus clause. Consider the law that metal will expand as it is heated. This only turns out to be true if pressure is constant, if the metal continues to exist (which may not happen if, say, a steel tube is heated to $10,000 \mathrm{~K}$ ), and a number of other factors. We might be able to enumerate these non-extant conditions individually, but for some laws there may be an infinite number of clauses that are required to stand in for a single ceteris paribus clause if we want to avoid reference to non-entities.

Ceteris paribus clauses and how our ontology will account for them will be dealt with when we discuss laws of nature in Chapter 4. For now, it is enough to note that eliminating negative properties from our ontology comes with the burden to explain such clauses in a way that does not use negative properties.

Historical properties are not properties that an object has immediately, but instead which it only has in virtue of having had a certain history. For instance, if I take two quarters, put one in my left pocket and the other in my right pocket, and then hand them both to you, one of those quarters has the historical property of 'having been in my right pocket' while the other has the historical property of 'having been in my left pocket,' even if they are otherwise qualitatively identical. To be clear, the question is not one of whether or not two objects can be different as a result of their histories, but rather whether or not the history of an object is itself is a property we ought to admit into our ontology.

Perhaps the strongest argument for the admission of historical properties into our ontology comes from four dimensionalism, wherein the true 'object' exists not only in space, but also in time. On four dimensionalist theories, the historical properties of an object are present in it as the object is literally extended over that history. On a non-four dimensionalist theory, historical properties may be more easily eliminated from one's 
ontology as they do not exist in the present moment. If one's theory of time says that past moments are not metaphysically real, then while the properties that existed at those past moments existed at the time they were the 'present,' they do not exist as past moments. This allows one to eliminate them harmlessly. But for the four dimensionalist those past moments still exist and thus, historical properties still exist in an important sense. However, even in this case the historical properties of an object are only present at those moments at which it 'is' (in a four-dimensional sense) at those times. Take a fourdimensional object $\mathrm{O}$ which exists at times $\mathrm{t}_{1}$ and $\mathrm{t}_{2}$. Let's assume that $\mathrm{O}$ has property $\mathrm{P}$ at $\mathrm{t}_{1}$ but does not possess it at $\mathrm{t}_{2}$. It is true of $\mathrm{O}$ that it has property $\mathrm{P}$, as it has $\mathrm{P}$ at $\mathrm{t}_{1}$ and $\mathrm{t}_{1}$, as a three-dimensional timeslice of $\mathrm{O}$, is a part of $\mathrm{O}$. But it is not true that $\mathrm{O}$ has $\mathrm{P}$ at $\mathrm{t}_{2}$ in any sense. Consider an analogous three-dimensional problem. Say that my desk has a crack in one of its right legs. This would mean that the desk has a crack in it, but it does not mean that the desk has a crack in its left leg. ${ }^{121}$ That one spatial region of the object has a property does not by itself indicate that other parts of the object contain the property. Similarly, the mere fact that an object had a property at a prior time does not say anything about the properties it has now, save for whatever properties are necessary for contiguity. So while historical properties exist in some sense on a four dimensionalist picture, they exist only at their prior point in time and not in the present. This means that there are no true historical properties for the four dimensionalist: just properties indexed to different times. As such, historical properties can be eliminated, although for the four dimensionalist

\footnotetext{
${ }^{121}$ The left leg does have a relational property of belonging to a desk with a cracked leg, but this is not a fact of the left leg itself. If the left leg were removed and affixed to a new desk, it would lose this property provided that the new desk was not cracked.
} 
it is important that properties be indexed to a particular time.

\subsubsection{Determinables and Determinants}

Finally, something must be said about determinable and determinate properties. Consider the properties of 'having a negative charge' and 'having charge.' The former is a case of the latter. Yet it appears that an electron has both properties. A constitutive trope theorist ought to be committed to determinate tropes, but ought they to admit of determinable tropes as well? An argument will be presented for eliminating determinables from our ontology, or at least not giving them the status of tropes. But we may need to include determinables in our ontology, given our third desideratum. Many scientific lawstatements refer to determinable properties, not determinate properties: Newton's second law, for instance, mentions the determinable properties of force, mass, and acceleration and not the determinate properties of specific forces, masses, and accelerations. Given our third desideratum, we must account for determinables in a way that allows for ontologically grounded laws.

One version of the argument to remove determinables from one's ontology follows. An object never has only determinable properties. An object cannot merely have velocity: it must have a particular velocity. It cannot simply have color: it must be of a specific hue and shade. ${ }^{122}$ Objects possess determinate properties, and these determinate properties belong to one or more determinables, but they do not possess determinable properties simpliciter.

\footnotetext{
${ }^{122}$ For emergent tropes, this leads to many tropes of a single determinable excluding one another. Often, if the relations between constitutive parts are sufficient for trope A, and trope A and B are of a kind, trope B will not be able to emerge as a matter of contingent fact. This is why many tropes exclude each other: for instance, an object cannot be both $5^{\prime}$ long and 6' long in the same dimension.
} 
Further, a property can belong to more than one determinable. Red is a color, a primary color, and a warm color. If we eliminate determinable properties in favor of the determinate we reduce the number of properties and the number of kinds of properties. This means that given our fourth desideratum, we ought to eliminate determinables as genuine properties (tropes), all else being equal.

If we are to eliminate determinables as tropes while giving them some role in our ontology, it may help to define what makes a property a determinable property rather than a determinate property. I believe a working definition is that a determinable is related to its determinate by differing only by specificity but not by kind. For instance, both the properties of being 5 ' tall and 6 ' tall fall under the determinable of height, but while these two determinate heights differ from each other by specificity, they are not different in terms of the kind of height. In this way, the determinable property is equivalent between the two heights even though the determinate is different. Compare this to the properties of being $5^{\prime}$ tall and 100 degrees Celsius. These two differ by kind: the height-determinable of the first property and the temperature-determinable of the second property are not interchangeable. ${ }^{123}$ They may yet share a very broad determinable of 'being a property' but qua height or temperature they do not share a determinable.

We can use this to solve our earlier dilemma by eliminating determinable properties from our ontology. Determinables are not tropes, but rather categories which signify that determinate tropes within the category differ only with respect to specificity. Provided that the specific differences and similarities are then grounded ontologically, we will have a

\footnotetext{
${ }^{123}$ It may be that all properties share some determinable, such as the property of "being a property" or even "being a trope." However, even with respect to these determinables, they do not differ with respect to being a case of the determinable in question.
} 
basis for grounding scientific law-statements which feature determinables ontologically. This is not yet a full solution to the problem: I have yet to show that similarity is grounded ontologically for constitutive trope theory. But it is a provisional solution, which by the end of this chapter will be a full solution.

\subsection{Objects}

Now that we have some idea what sorts of properties are admitted into this ontology, there needs to be some discussion of what it is for something to be an object. Simply stated, an object on this theory is nothing over and above a collection of compresent ${ }^{124}$ tropes. However, because tropes are dependent on their constitutive parts, objects will be indirectly dependent on the constitutive parts of the tropes which compose them. As the existence and location of each trope is due to its constitutive parts, tropes are compresent when their constitutive parts are identical, have spatial overlap, or some combination of the two. So while an object is nothing more than a compresence of tropes, the existence of the object is not entirely dependent on the compresence of these tropes but rather is dependent on there being relations between the parts ${ }^{125}$ such that some of the emergent tropes which compose the object are still extant.

\footnotetext{
124 What compresence amounts to on this theory will be discussed in more detail once we turn our attention to relations.

125 The ontological status of relations, and questions such as their spatial location and their presence with respect to their relata, will be considered later in this chapter.
} 


\subsubsection{Change and Persistence}

Given the above, our theory allows objects to lose some of their tropes without losing the object as a whole. It can help to demonstrate this. Say that we have a particular object A which contains three tropes, X, Y, and Z. The constitutive parts of A's tropes we will call P. Each trope is then dependent on a collection of relations between its constitutive parts sufficient for the existence of the trope. For simplicity, $X$ will be dependent on $\mathrm{Rx}_{\mathrm{X}}(\mathrm{P}), \mathrm{Y}$ will be dependent on $\operatorname{Rr}(\mathrm{P})$, and $\mathrm{Z}$ will be dependent on $\mathrm{Rz}_{\mathrm{Z}}(\mathrm{P})$. Say that the set of relations between the parts changes such that Rx and Ry continue to hold, but Rz no longer does. Provided there are no other relations among $\mathrm{P}$ sufficient for $\mathrm{Rz}, \mathrm{Z}$ will cease to exist. However, by stipulation $\mathrm{Rx}$ and $\mathrm{RY}$ continue to exist and thus, $\mathrm{X}$ and $\mathrm{Y}$ continue to exist, as do all parts P among which the initial relations held. As such, A still exists despite the loss of $\mathrm{Z}$ as the component parts held together enough for some collection of A's compresent tropes to continue existing. Similarly, an object may gain properties without becoming a different object.

The question then arises as to what the necessary minimum conditions are for an object to exist and what does it mean for an object to exist over time. I will start by diving right into a tough case. Consider an object $\mathrm{X}$ at $\mathrm{t}_{1}$, which for the sake of simplicity will consist of 4 compresent tropes: A, B, C, and D. The tropes A, B, C, and D are all emergent on the relations of 4 parts: $\mathrm{p}_{1}, \mathrm{p}_{2}, \mathrm{p}_{3}$, and $\mathrm{p}_{4}$ as follows:

$\mathrm{R}_{\mathrm{AB}}\left(\mathrm{p}_{1} \& \mathrm{p}_{2}\right)$ is sufficient for the existence of $\mathrm{A}$ and $\mathrm{B}$.

$\mathrm{R}_{\mathrm{CD}}\left(\mathrm{p}_{3} \& \mathrm{p}_{4}\right)$ is sufficient for the existence of $\mathrm{C}$ and $\mathrm{D}$.

$\mathrm{RBC}_{\mathrm{B}}\left(\mathrm{p}_{2} \& \mathrm{p}_{3}\right)$ is sufficient for the existence of $\mathrm{B}$ and $\mathrm{C}$.

No other relations between $\mathrm{p} 1-4$ are sufficient for A, B, C, or D. 
Now let's assume that the relations between p1-4 change such that $\mathrm{R}_{\mathrm{AB}}$ and $\mathrm{R}_{\mathrm{CD}}$ each hold individually while $\mathrm{R}_{\mathrm{BC}}$ ceases to hold. In this transformation, A and B remain compresent with each other and $C$ and $D$ remain compresent with each other but the composite of (A\&B) is not compresent with the composite of (C\&D). As they are not compresent, we now have 2 objects, which we will call $Y$ and $Z$. The question is: which of $Y$ and $Z$ is $X$ ? It seems undecidable: both $\mathrm{Y}$ and $\mathrm{Z}$ appear to have equal claim to 'being' $\mathrm{X}$.

We might think that $\mathrm{X}$ simply does not exist anymore and worry little about any ontological consequences of this. After all, objects on this account are nothing over and above collections of tropes, so it is not surprising that if those tropes cease to be compresent that the object should cease to exist. But this becomes a problem given our earlier account of change. If an object can gain or lose tropes while still being 'the same object' then why, if it loses tropes in such a manner that those tropes go on to constitute new objects, should the object then cease to exist? Having them both be X, of course, runs into obvious problems of identity of dissimilar objects. $\mathrm{Y}$ and $\mathrm{Z}$ are non-identical with each other, so it is not clear how they could both be identical with X.

\section{$\underline{3.2 .2 \text { Identity of Objects }}$}

Answering this puzzle requires that we solve the problem of what makes an object the same object over time. In the case of objects whose tropes are emergent, we must ask what makes an object the same object over time. It cannot be merely occupying a particular spatial region: objects can change location. Nor can it be that the object retains all of its 
properties, unless we throw away the idea that an object can change. Yet compresence (a spatial relation) of tropes is all an object is.

Consider a case of change: a child growing into an adult and then later becoming a corpse. These changes may be slow, or they may be abrupt. The line between a child and an adult is fuzzier than the line between a human being and a corpse, although there may be cases, such as the brain dead, where this line becomes difficult to draw. Yet children and adults have different mental and physical characteristics, as do corpses and human beings. Whether or not an object falls into one of these categories depends on the properties it has at that time. Here I intend to utilize a strategy in line with Burke's dominant sortal theory. ${ }^{126}$ Children, adults, and human beings are all categories to which an object can belong in virtue of its properties. An object may belong to more than one sortal, and whether or not it is a member of that sortal is a matter of whether or not it has those properties at a particular time. The object then continues to be an object of that sort so long as it maintains the properties for membership in that sortal. In our earlier puzzle, this means that $\mathrm{X}$ would be an A-sort of object and a C-sort of object and an (A\&C)-sort of object, while Y would be an A-sort of object but not a C-sort of or (A\&C)-sort of object and Z would be a C-sort of object but not an A-sort of object or an (A\&C)-sort of object. Change for an object is a matter being of a sort: if an object at rest gains momentum, it becomes a moving sort of object. If it then comes to rest again, it is no longer of this sort. In the above transformation, then, $\mathrm{X}$ becomes $\mathrm{Y}$ and $\mathrm{Z}$. $\mathrm{Y}$ is an A-sort and B-sort of object and its

${ }^{126}$ Michael Burke, "Preserving the Principle of One Object to a Place: A Novel Account of the Relations Among Objects, Sorts, Sortals, and Persistence Conditions," Philosophy and Phenomenological Research, Vol. 54, No. 3 (Sep., 1994), pp. 591-624. 
constitutive parts were once a part of $\mathrm{X}$ and were what made $\mathrm{X}$ an $\mathrm{A}$ - and $\mathrm{B}$-sort of object, and similar statements can be made for $\mathrm{Z}$, and nothing more is to be said.

Let's consider another relevant case: a case where an object splits into two objects with identical properties. Assume we have an object $X$ with just 2 compresent tropes, $A$ and $\mathrm{B}$ and parts $\mathrm{p}_{1}, \mathrm{p}_{2}, \mathrm{p}_{3}$ and $\mathrm{p}_{4}$ where the following is true:

$R_{1}\left(p_{1} \& p_{2}\right)$ is sufficient for $A$, and is also sufficient for $B$.

$\mathrm{R}_{2}\left(\mathrm{p}_{3} \& \mathrm{p}_{4}\right)$ is sufficient for $\mathrm{A}$, and is also sufficient for $\mathrm{B}$.

R1 and R2 are compresent relations, meaning they occupy the same spatio-temporal area.

This is possible, as a trope's existence may be overdetermined. Now postulate that, as in our previous example, $\mathrm{p} 1-4$ change such that R1 and R2 are no longer compresent, with new objects $\mathrm{Y}$ and $\mathrm{Z}$ corresponding to $\mathrm{R} 1$ and $\mathrm{R} 2$. In this case, objects $\mathrm{Y}$ and $\mathrm{Z}$ both lay claim to all of the same kinds that $\mathrm{X}$ lays claim to, but are distinct objects: if $\mathrm{Y}$ further transforms to obtain a new trope $\mathrm{C}$, we do not expect this to have any effect on $\mathrm{Z}$. So the question of which of $\mathrm{Y}$ or $\mathrm{Z}$ is the "original" object $\mathrm{X}$ does not have an answer: both fit the criteria for having been X originally. But we can say whether they share a kind with $X$, and whether or not they do so in virtue of a shared history of parts. This question we can answer. So objects changing is then reduced to a matter of tropes becoming compresent or losing compresence, of coming into being and falling out of being, in relation to the movement of the parts on which they supervene. Asking whether or not an object is 'the same' object over time is reduced to questions about whether or not an object has retained a kind in 
virtue of the tropes that compose it, rather than there being some primitive fact about identity.

This account of change and the identity of objects is similar to that given by Peter Simons, but it is importantly distinct. ${ }^{127}$ Namely, this is because Simons has what he deems a 'nuclear theory' of tropes. Simons makes a distinction between those tropes which are essential for an object's continued existence and those which are accidental and says that an object can change yet persist by losing and gaining accidental tropes so long as it retains its essential tropes. The essential tropes are then what make an object the same object over time. Constitutive trope theory does not have an accidental vs. essential trope distinction. Instead, as long as constitutive parts of an object that are sufficient for the continued existence of some of its tropes still exist, the object still exists. Object identity on constitutive trope theory is a matter of identity qua a property or collection of properties, and while we generally predicate those properties or collections of properties that we take to be important, from a metaphysical standpoint these distinctions are arbitrary on constitutive trope theory.

\section{$\underline{\text { 3.2.3 Pseudo-objects }}$}

Just as we eliminated some properties as pseudo-tropes, we may also eliminate some objects from our ontology as pseudo-objects. And we may do so using the same principles. For instance, having eliminated aggregates as tropes, we can eliminate objects whose only distinct properties are aggregate properties. Consider the case of a heap of

\footnotetext{
${ }^{127}$ Simons, Peter. "Particulars in Particular Clothing: Three Trope Theories of Substance." Philosophy and Phenomenological Research 54, no. 3 (1994): 553.
} 
sand. Many if not all of its properties: its weight, its conductivity, its location, all amount to nothing more than the aggregate of the properties of the grains of sand that make up the heap. If the heap will have any property that might be taken as an emergent property, rather than an aggregate, it would be its shape. Spatiotemporal properties such as shape present unique difficulties to be discussed later. But if we assume that all of the properties of the heap are aggregate properties, then the heap itself has no ontological significance in this account: it is a mere collection and not a genuine object. Other objects may be eliminated on similar grounds. For instance, an "object" distinguished from its parts only by historical properties or negative properties will be eliminable in the same manner that objects defined only in terms of aggregates are eliminable, as these were also eliminated from our ontology.

\subsection{Relations}

Now that we have tropes and objects in our picture, we can address relations. While properties are 'monadic' in that they hold of a single object, relations are 'n-adic' in that they hold between entities. Relations do not have to be binary: relations can have 3-places, 4-places, or some other number of places. For the sake of simplicity, most of the examples I will use will involve two-place relations. Relations exist due to the distinguishing features of their relata. For instance, if a car is moving at $90 \mathrm{mph}$ and a person is walking at $2 \mathrm{mph}$, the car has the relation of "faster than" to the person. Relations, of course, need not be of differences between their relata. If a boat and a car are both moving at $90 \mathrm{mph}$, they bear the relation of "moving at equal speed" to each other. 
There are two sorts of relations that need to be considered. Internal relations and external relations. Internal relations hold between properties or objects merely because of the intrinsic nature of those properties. For instance, if I am 6' tall and my friend is 5' tall, that I bear the relation of "taller than" to them is entirely because of the respective natures of our height properties. Compare this to the relation of "being inside of," such as if I am inside of my house. This relation of me being inside of the house is not a fact about my intrinsic properties. Rather, it is a fact about my position in the world relative to the house. Thus, this is an external relation.

Many philosophers have viewed internal relations as not requiring any special metaphysical explanation, with Armstrong referring to them as a "ontological free lunch." I am inclined to agree. We do not need to posit relations as entities to explain how I am taller than my friend given that I am 6' tall and they are 5' tall. The relation is entirely explained by the character of the properties we each possess individually. External relations are not so easy. The relationship between me and my desk where I am "in front of" my desk is not explained by my intrinsic properties or those of the desk. But something must ground this relation ontologically if it is to exist. As we are working with a trope theory, I will posit a specific species of trope, relational properties, to ground external relations of this sort.

At first, we might want to think of relational properties as a kind of emergent property. But there is an important distinction in that while the constitutive parts of an emergent property are a sufficient condition for the existence of the property, the relata of an external relation are a necessary condition for the existence of that relation. If James is older than Tom and his only sibling, then if James did not exist then no one would stand in the 'older brother' relation to Tom. Even in the case where Tom has another older brother, 
that is a different relation than his relation to James. To use a physical case, if modern science is correct and nothing can move faster than the speed of light in a vacuum, then nothing stands in the 'faster than' relationship to any photon in a vacuum. So what distinguishes relations from other emergent properties is that while the constitutive parts of a property are a sufficient condition for that property, the relata of a relation are necessary for that relation.

Now that we have something of a handle on relations and relational properties, we can examine them more fully. First, we must ask what are the allowed relata of relations: are they tropes, objects, or both? Second, we must ask what sort of thing a 'relational property' is on this ontology. Given that this is a single-category ontology, there are four potential solutions one could posit: relational properties may be a kind of trope, a kind of object, ${ }^{128}$ they may be ontologically grounded in tropes, ${ }^{129}$ or they may be eliminated from the ontology. I will immediately reject the last of these four answers. Both compresence and constitution are present in this picture and both can accurately be described as relations. Further, as the relations of compresence and constitution are not internal relations, there must be some relational property to ground them. So we cannot eliminate relations or relational properties from the ontology entirely as this would require some new account of core pieces of the theory. To decide between the other three answers to this second question, we must know what the relata of relations are, particularly external relations.

As our theory currently only has tropes and objects standing in relations, we must ask what are the legitimate relata of relations: tropes or objects? This is best handled by

\footnotetext{
128 This answer is only plausible if it turns out that to explain a relation we must have a collection of compresent tropes.

${ }^{129}$ Recall that this is, broadly, the answer that this theory gives for the question, "What is an object?"
} 
dividing this into two separate questions. The first is whether or not tropes are legitimate relata of relations, and the second is whether or not objects are legitimate relata of relations.

\subsubsection{Relata}

Prima facie, there is a reason to admit tropes as legitimate relata of relations: tropes are the ontological foundation of this theory. If tropes are the sort of thing that can ground objects, then they are capable of grounding something, and so may also be able to ground relations. Further, we use relations between tropes to talk about the world. Take a statement such as "it is colder outside than inside." If we take temperature to be a trope, this appears to be a statement which demonstrates a relation between two tropes: namely, that there is a particular difference between the temperature-trope inside the room and the temperature-trope outside the room such that it is colder outside. We can compare the brightness of two colors, the charge of two particles, or the height of two objects without referencing any of their other properties. So it seems that tropes are legitimate relata of relations.

However, in the case of tropes and their relations, each appears to be an internal relation. The fact that it is colder outside than inside supervenes on the nature of these distinct temperature properties and is entirely explained by them. There are a few exceptions to this, however. For one, the relationship of constitution between a trope and its constitutive parts is not an internal relation. This is because the trope could, in principle, be sustained by different constitutive parts as discussed earlier. As such, it is not a part of that trope's nature that it exist due to the existence of the particular constitutive parts that 
in fact sustain it. Compresence is another external relation in which tropes feature. For many compresent tropes, that those particular tropes are compresent with each other is not a fact which supervenes on the nature of those tropes taken individually. So we have at least two external relations in which tropes feature, meaning that tropes can be the relata of external relations as well as internal relations.

But there is a problem with respect to objects. Compresence is a relation between tropes: the relation of being spatially co-located. If compresence is an external relation, then for a one-category ontology we will need a relational property that grounds it. As an object is just a compresence of tropes, then being an object is either grounded in a relational property (a compresence-relational property) or it is identical with such a property. In the latter case, we end up with the (perhaps counterintuitive) conclusion that objects themselves are a species of relation.

And there are reasons to favor this, as it blocks a potentially vicious regress. If the object is not the relational property of compresence but is instead grounded in that property, we must ask if the relational property of compresence is itself compresent. If it is not, then it is not clear where it is located. If it is, then we are off on a vicious regress. For the compresence relation will then be a the relatum of a second compresence relation between itself and its relata. And the second will lead to a third, and so on and so on. But if the object is identical with the compresence relation then we may block this regress, as it is part of the nature of the object that it be compresent with the tropes it bundles. This makes the compresence relationship for objects an internal relation and thus we need not posit any further relational properties to ground it. So there is good reason to identify objects as identical to the compresence relation between the tropes that they bundle. On this view, 
then, a particular object is identical with the bundling of the particular tropes that compose it. So can an object, as a relation between tropes, be a relata for another relation?

On the sort of intuitive view utilized above, it seems they ought to be. Take a case where Alice and Bob are in two separate parts of the world. For Alice, it is 70 degrees inside and 50 degrees outside. For Bob it is 65 degrees inside but 10 degrees outside. For both Alice and Bob, it is colder outside than it is inside, but for Bob the discrepancy is wider than it is for Alice. This seems to be a case of there being a relation between relations. Further, such statements are used regularly in scientific research and theories. Given that one of our desiderata is to give a metaphysical ground for scientific practice, it seems that relations are able to be relata themselves. As objects are a kind of relation according to this theory, it seems that objects may also be the relata of relations.

Knowing this, what sort of entity is a relational property on this account? The simplest answer is to admit relational properties as tropes, but tropes of a particular kind. We have already made a distinction between emergent tropes and foundational tropes, as emergent tropes are supervenient on other complexes of tropes while foundational tropes are not. Relational properties cannot be foundational tropes as they require other tropes for their existence, but if they are emergent tropes, on what do they depend? The answer is just their relata. But as previously discussed, relational tropes are unlike emergent tropes in that the relata of a relation are existentially necessary rather than existentially sufficient for the existence of that relation. This does not mean that there are no contingent relational facts: which relations emerge from a given set of tropes will be largely dependent on contingent circumstances. But it does mean we have a third species of trope: relational properties. Similar to the relationship between emergent tropes and their constitutive 
parts, relational properties are dependent on their relata for their existence. But the relationship is distinct: constitutive parts are sufficient for the existence of their emergent tropes, while relata are necessary for the existence of their relations.

\subsubsection{Compresence Relations and Objects}

As stated earlier, if each object is just a compresence of tropes and compresence is a relation, this means that objects are relational tropes. This makes this theory of tropes a true one-category ontology thus far, as no entities need be postulated that are not themselves tropes, including the objects which we normally take tropes to belong to.

But even if objects turn out to be simply a kind of relational trope on constitutive trope theory, objects have a special place with respect to emergent tropes and thus, this relation deserves some extra explanation. To start with, compresence is an irreflexive, symmetric, transitive relation. If trope $A$ is in a relation of compresence with trope $B$, then $B$ is in a relation of compresence with trope $\mathrm{A}$, and $\mathrm{A}$ and $\mathrm{B}$ will be non-identical. This also means that if a trope is in a compresence relation with another trope, each trope is also compresent with all other tropes with which the other shares a compresence relation. If Trope $A$ is compresent with trope $B$ and trope $B$ is compresent with trope $C$, then trope $A$ is compresent with trope C. This collection is the 'bundle' of tropes. As all tropes in a bundle are compresent with all other tropes in that same bundle, a trope cannot belong to more than one bundle. If Trope A were to belong, per impossible, to Bundle $\mathrm{X}$ and Bundle $\mathrm{Y}$, then $\mathrm{X}$ and $\mathrm{Y}$ would have to share compresence by the transitivity of the compresence relation.

As an object is this compresent bundle, a single non-relational trope cannot belong to more than two objects. 
But this leaves the question of object parts and compresence relations. Take a set of constitutive parts, $\mathrm{P}$, each of which is an object which give rise to one of a number of tropes $\mathrm{T}$ in an object $\mathrm{O}$. Each of the tropes in $\mathrm{O}$ belongs to $\mathrm{O}$ in virtue of existing in a compresence relation, which it does due to the spatial overlap between $\mathrm{P}$ and $\mathrm{O}$. If $\mathrm{A}$ is an object in $\mathrm{P}$, doesn't this create a contradiction, as A must, as an object in P, overlap with 0 ? This will only be so if the tropes of $\mathrm{O}$ and $\mathrm{P}$ exist in a relation of compresence. But if we assume this, we can derive a contradiction if parts can have contrary properties, which it appears they can and do. An atom will have as its constituent parts some number of protons and electrons. An object cannot be both positively and negatively charged at the same time. Yet protons carry a positive charge and electrons carry a negative charge. If the atom is compresent with its parts, then the atom has contrary properties. It must instead be that compresence is a relation between tropes with identical spatiotemporal locations. The spatiotemporal location of the electrons and protons of an atom is entirely contained by the spatiotemporal location of the atom, but they are non-identical: the atom exists in places the spatiotemporal location does not. This gives us the compresence relation: an irreflexive, symmetric, transitive relation between tropes which occupy identical spatiotemporal locations.

\subsection{The Problem of Universals}

Now that we have given answers to what makes something a trope, object, and relation, we are able to attempt to answer the Problem of Universals. That is, how it is that two tropes can be similar to one another? There are two kinds of similarity we will examine. Recall that in earlier discussion, the distinction between determinables and 
determinates was made, with two determinate tropes which share a determinable sharing this determinable in common, differing only in terms of how they specifically instantiate that determinable. This will be one form of similarity that we will examine. The other will be the case of "total" or "complete" similarity, where two tropes are as similar as is possible for distinct properties to be.

\subsubsection{Similarity}

With the distinction between determinates and determinables, and what it means for a determinate to fall under a particular determinable, we may now attempt to answer the problem of universals. That is, we may answer the question of what it is that makes two tropes similar to each other. For the tropes most similar to each other will always be of a kind: two mass tropes or two charge tropes will always be more alike than a mass trope and a charge trope will be like each other. However, while this works as a minimal condition for two properties being similar, it is not sufficient for describing similarity: A 5' length of rope is more similar, ceteris paribus, to a $6^{\prime}$ length of rope than a $200^{\prime}$ length of rope. So how can we account for similarity by degree, rather than the minimal similarity of belonging to the same determinable?

As we saw in Chapter 2, one weakness of some trope-theoretic accounts is their lack of an analysis of similarity or resemblance, with Campbell and Martin both taking resemblance to be a primitive. As Bacon ${ }^{130}$ notes, one option is to take resemblance to be an equivalence relation. There are two problems for a trope theorist who takes this route, however. The first is spelling out what it means for two tropes to be equivalent. The second

\footnotetext{
${ }^{130}$ John Bacon, “A single primitive trope relation," Journal of Philosophical Logic 18, No. 2 (1989): p.142.
} 
is that while similarity is often a more-or-less notion, equivalence is not. Two objects can be more-or-less similar, but they cannot be more-or-less equivalent.

On one level, we can spell out what is meant by an equivalence relation. To be in a relationship of equivalence is to be in a relation that is reflexive, symmetric, and transitive. But this alone does not tell us what it is for two tropes to be equivalent qua similarity. One version of this would be logical equivalence. Immediately, we are faced with the problem that logical equivalence is a matter of propositions being equivalent, but tropes are not propositions. We may give logical equivalence its best argument here by then saying that it is a matter of how tropes feature in propositions in referentially transparent cases. That is to say, if a reference to one trope in a statement can be replaced with a reference to another trope without altering the truth of the statement, they are equivalent qua that statement. And if two tropes are truly equivalent, we may do so for any statement regarding the tropes in question. Take the property of being red. If the redness of a stop sign and the redness of a coke can are equivalent, we may then take any statement regarding the redness of the coke can and the redness of the stop sign and interchange them. So if the above equivalence holds and the statement, "The stop sign is a brighter red than this apple" then if we replace 'stop sign' with 'coke can' in the above statement, its truth value will remain the same.

There are two problems with this, one minor and one major. The minor problem is that similarity becomes about statements, meaning that statements will feature prominently in a trope ontology that accepts this version of equivalence for similarity. There are reasons to be suspicious of this. ${ }^{131}$ The blow can be softened by stipulating that

${ }^{131}$ Some of these reasons were examined more thoroughly in our examination of Lewis' Best Systems Analysis in Chapter 2. 
while it is true that if two tropes are equivalent, then any statement about one of these tropes, it may be replaced by the other with no change in truth value, this is not what makes two tropes similar. Rather, it is a description of how statements relate to similarity in a trope ontology. I think this strategy has flaws, but I will let that matter drop as I believe another issue is much more pressing. And that is that there are certain statements for which no two distinct tropes will ever be able to replace each other: those of belonging to an object. To use our earlier example-tropes, "The redness of this stop sign is a property of the stop sign," will be true for the redness of the stop sign, but not the redness of the coke can. In fact, the trope theorist must deny this or their theory will collapse into realism about universals. This is true even in the case where there would be no discernable difference between the objects if it were (per impossible) true of the stop sign that it possessed the coke can's redness. So I do not think that any of these strategies work, and we must examine other avenues for understanding similarity.

\subsubsection{Indiscernibility}

But the above notion of indiscernibility may prove more useful to us. That is, that what makes two tropes similar is that there are no relevant differences between them. This is not to say that there are no differences, but that there are no differences which are relevant to the similarity relation. ${ }^{132}$ This overcomes several problems for the earlier 'equivalence' account. First, while logical equivalence is an all-or-nothing concept, having differences is not. Two things can be more or less different to each other than two other

\footnotetext{
132 To keep this from being trivial, one must determine which differences are relevant or irrelevant. This is what distinguishes different versions of this account.
} 
things. Second, talking about differences between properties does not require us to talk about propositions, meaning that propositions need not be added to our ontology. Third, and perhaps most importantly, we can ground the sorts of statements that made equivalence seem appealing in indiscernibility. What makes the terms 'coke can' and 'stop sign' interchangeable in our above example is the indiscernibility of their color properties.

One might object here. If similarity is analyzed as there being no relevant difference between two properties, we seem to have just traded primitives: 'similarity' for 'difference.' One might even charge this account with being circular as in our ordinary language, similar and different may be used as antonyms. I will accept that I have chosen differences as a primitive of my theory rather than similarity, but I believe there are principled reasons for thinking it more primitive. Consider for a moment two things which are very different such as a tune of music and a color. If you are asked how these are different, all you can really do is appeal to the nature of each and point out that there is a distinction between them. If your interlocutor did not understand how they were different at that point, there would be little you could say to them to show them. Even for two very similar things, such as two shades of red, there is little else you can do to explain how they are different. This does not show that there is no potential analysis of how it is that two things can be different, but such an analysis may be difficult to perform because of how fundamental it is to our understanding of the world. At least at this time, I am not prepared to offer such an analysis. And as I am not offering an analysis of what it is for two things to be different, the charge of circularity cannot be brokered at this time. Only if I provided an analysis of difference in terms of similarity could such an account be offered, and I do not have such an account of difference for analysis. 
One plausible version of this, but one that I will reject, is that two tropes are maximally similar if the only differences between them are spatiotemporal. That is, any two tropes are maximally similar iff only their spacetime locations distinguish them. As discussed in Chapter 2, Martin seems to accept this version of indiscernibility when discussing resemblance. While I think this is a plausible version, I think that making this our primary criterion for similarity complicates the account significantly. This is because basing our criteria for similarity primarily on spatiotemporal location requires that we have an account for spatiotemporal location. As we will see, such an account is problematic, in no small part because of the question of whether or not having a particular spatiotemporal location is itself a trope. Further, this requires our trope theory to commit to some such account, which I will argue later it has no reason to do. While spatiotemporal location is a plausible candidate for our difference-maker between tropes, if we have a less philosophically fraught option then we should take it.

In addition, there may be technical reasons to suspect spatio-temporal location is not the true difference-maker for tropes. Consider a case where there is water pressure and there is air pressure (perhaps on different objects) where both are producing the same amount of force. It seems that there are differences between the water pressure and air pressure that are not captured merely by their spatiotemporal location or factors directly related to their spatiotemporal location. For instance, facts about how these properties are instantiated might imply differences in other properties or relations in the objects that instantiate them. For instance, the "burst pressure" or pressure at which a container will rupture, is often different for air and water pressure due to differences in the movement of 
air particles vs. water particles. These differences are difficult to cash out entirely in terms of spatiotemporal differences.

And it is the above notion of the difference in instantiation that I will submit as a better alternative to spatiotemporal location as a difference-maker for maximally similar tropes. In other words, the only difference between two maximally similar tropes is that each has different constitutive parts. This not only has advantages in technical cases such as the one above, it also has the advantage of explanatory simplicity. In the case of emergent tropes, we already have an account of what instantiates them: for each emergent trope there is some relation between its constitutive parts on which it supervenes. As the dependence of emergent tropes on their constitutive parts is already a part of our account, the account need not require an answer to any new problems to invoke it for our purposes here. There may be problems here for foundational tropes, as they lack the sort of supervenience that emergent tropes possess. However, I will have to bracket this issue until we discuss foundational tropes at the end of this chapter.

It is also noteworthy that in many if not all ${ }^{133}$ cases, grounding the difference between tropes in their supervenience gives us tropes that are spatiotemporally distinct. This is because the supervenience relation is a sufficiency relation. Consider the case of two sets of constitutive parts $\mathrm{P}$ and $\mathrm{Q}$, each of which exists in a relation $\mathrm{R}(\mathrm{aP})$ and $\mathrm{R}(\mathrm{aQ})$, where an $\mathrm{R}(\mathrm{aX})$ relation indicates one where $\mathrm{X}$ is sufficient for trope $\mathrm{A}$. As long as the parts overlap, there is only one A trope. This may seem like we are back to a spatial criterion, but the criterion is still one of constitutive parts, as the constitutive parts are a sufficient condition for A rather than a necessary condition for $\mathrm{A}$.

\footnotetext{
133 There is an interesting case here in the case of quantum superposition, which I will discuss in Chapter 5.
} 
As such, both sets of constitutive parts may ground the same A. This may seem counterintuitive as what makes two different tropes distinct is that they have a different set of parts on which they supervene, but in the case where a trope supervenes on more than one relation of constitutive parts there is no distinction. The case where P and Q are identical but $\mathrm{R}(\mathrm{aP})$ and $\mathrm{R}(\mathrm{aQ})$ are not may help. That is, the case where there is only one set of parts but more than one relation between those parts that is sufficient for A. Should the parts change such that $\mathrm{R}(\mathrm{aP})$ does not hold but $\mathrm{R}(\mathrm{aQ})$ still holds, A would still exist, and vice versa. Yet there is only one collection of constitutive parts that grounds A. The case is the same when there are two sets of parts each of which has a relation is sufficient for a particular A trope in a particular location. Each being sufficient leads to there only being one trope that is present. 134

We now have a maximal and minimal similarity distinction between tropes: two tropes are maximally similar iff the only difference between them is a difference in their instantiation, and the minimal condition for being similar if two tropes share a determinable.

\subsection{Problematic Properties}

Having given the basic theory of properties, objects, and relations for the theory of tropes, I will spend some time now on a few properties that are difficult and important cases for the theory. While there may be other properties that we should concern ourselves with, these properties are unique not only in that they present a challenge for our theory of tropes, but that they are in some way central to the ideas presented earlier.

${ }^{134}$ When we consider the case of quantum superposition in Chapter 5, we will see evidence for this claim. 


\section{$\underline{\text { 3.5.1 Accidental and Essential Tropes }}$}

We also need to say what it is about tropes that makes them accidental or essential to an object. To distinguish these, we say that an accidental property is one that an object possesses but is not a part of its essential nature. That is, we think that an object can lose a given property and have it replaced with another while still remaining the same object. Take the case of a particle with properties such as mass, spin, velocity, charge, etc. Some of these tropes may be had accidentally, while others are had essentially. How we distinguish between accidental and essential properties should, at minimum, help us to decide which properties are which.

Given our earlier account of change, where the question of whether or not X is 'the same object' is ill-formed, we might think that there is nothing more to be said about accidental and essential properties. We should ask not about the persistence of objects but of the properties of the object that are relevant to a particular distinction. However, more can be said than this because of the relationship between a trope and its constituent parts, at least in the case of emergent tropes. Take the properties of an isotope, such as its melting point, boiling point, and the Vanderwaals radius of its atoms. A given atom of that element not only has these 'generic' properties, but also others such as a particular velocity. Some of these tropes will be related to the constitutive parts of the atom in such a way that eliminating or replacing that property is not possible without eliminating or replacing every other property in the bundle: one cannot change the atomic weight of an atom of gold without changing the relations between its constituent parts in such a way that it loses all 
of its non-aggregate properties, but one can change the velocity of gold while allowing its other properties to remain stable.

This, provided with our earlier account of change, gives us a way to distinguish between the accidental and essential tropes of an object. The essential tropes are those where the elimination or replacement of the trope will eliminate every other trope in the bundle. The accidental tropes are those where eliminating the trope does not eliminate every other trope in the bundle.

\subsubsection{Spatiotemporal Properties}

As mentioned earlier, spatiotemporal properties may present a unique challenge for our theory. This challenge manifests itself for a few reasons. First, all empirical objects possess spatiotemporal properties. Second, in ordinary cases spatiotemporal properties appear to be aggregates. Short of there being a minimum length or minimum distance, it seems that any spatial or temporal property can be subdivided into a collection of like properties without any ontological loss. And even if there is a minimum length or distance, it would still appear that all non-minimal lengths or distances should then be aggregates. Third, as our theory will deal with compresent tropes when talking about objects, it seems some account of spatiotemporal properties is necessary as compresence seems tied to a particular point in space and time. As compresence occurs for a particular area of space and a particular duration, spatiotemporal properties are important for understanding compresence. It is for this reason I believe that not accounting for spatiotemporal properties adequately would be a mistake for a constitutive trope theory. Fourth, as one of our goals is to account for scientific laws and many scientific laws include statements about 
spatiotemporal properties, it seems that one of our desiderata requires us to give an account of these. Fifth, and perhaps more damningly, whether or not there is such a thing as a 'minimum length' or 'minimum duration' is its own philosophically fraught problem. And there may be good reasons to deny that there is such a 'minimum' for spatiotemporal properties. For there to be a minimal spatiotemporal property, there would have to be some length or duration beyond which it is impossible to divide any further, at least in the sense of physical possibility. However, even if this is the case it does not seem to be the sort of thing that one can discover about the world through a priori philosophical investigation. It might be that physics discovers that beyond a certain length or duration subdividing such spatiotemporal properties cannot occur: particularly if it turns out that spatiotemporal properties are not foundational. But even if this is so, it is suspicious that one might be committed to this from an a priori investigation into the nature of properties, rather than an empirical investigation into what, if anything, underpins spatiotemporal properties.

There are three sorts of spatiotemporal properties. The first such properties are properties such as length, breadth, and duration, that is to say, a spatiotemporal property that an object has in and of itself and independently of the world outside of the object. These are facts regarding the spatial and temporal dimensions an object occupies, such as occupying a 1" cube or existing for a period of 3 minutes. ${ }^{135}$ I will call these area-properties. Another such property is the property of having a particular location: being in New York City or in the year 1845. Accurate descriptions of these properties reference the outside world but do not directly reference objects in the world: the area where New York City is

\footnotetext{
135 It is notable that relativistic physics may come into play here: given sufficient velocity or gravity, two 1" cubes may appear to be of different lengths to an outside observer. However, internally, their lengths are stable.
} 
would exist without New York City, and saying an object was in that location is not to identify it with the position of that object. I will call these location-properties. Finally, some spatiotemporal properties are between objects: the distance between the floor and the ceiling, or between the start of World War II and today. I will call these spatiotemporal relations. ${ }^{136}$

Area-properties are emergent tropes and one of the simplest to account for on this theory. The size and duration of an object that is not entirely composed of foundational tropes $^{137}$ supervenes on the relations between its component parts. This is because the emergent trope, as discussed earlier, exists at the location of its constitutive parts. As such, emergent tropes, and the objects in which they are compresent, must exist at the location of their constituent parts. A chair has the size it does because of the wood that composes it and lasts for as long as the wood that composes it maintains the relations that allow for the existence of the chair. Spatiotemporal relations are similarly simple to account for, being relations of distance between location-properties. If my desk is a foot from the wall, that expresses a relation between the location-property of my desk and the location-property of the wall. That leaves only location-properties, which will turn out to be more difficult to account for.

The primary difficulty for location-properties is that, unlike area-properties, the location properties of each of an object's constitutive parts are not merely sufficient for the

\footnotetext{
${ }^{136}$ Some philosophers have denied one of these properties or attempted to reduce one to the others. Here I will treat them as separate so as to provide some idea of how each will work independently of any such theory about spatiotemporal properties which does not directly derive from this theory. The compatibility of such theories with this one is left as an exercise to the reader.

${ }^{137}$ Even if we do not doubt that there are foundational tropes, we might think that no such tropes are compresent, and so there might not be any objects which are composed of them. Further, it is unclear whether such objects would have area-tropes, so the point may be moot.
} 
object's location property. Rather, the location-property of an object is identical to the location-property of its constitutive parts. The location of a lake's water molecules is not merely sufficient for the lake's having a determinate location: the location of the lake at a given time is identical to the location of the water molecules that compose it at that time. So unlike the case of area-properties, where our standard account provides some explanation to the existence of the property, location-properties cannot be explained merely by supervenience.

There are two facts about location-properties that may help us here. The first is that location-properties are possessed by other properties. We can talk about the location of the color of an object or the time at which an apple is decaying. Even pseudo-tropes such as aggregates have location-properties. The second is that empirically, every object has a location. There is a place and time at which an object exists, if it exists. A theory of universals may posit some form of 'platonic heaven' for its universals to reside in, though not all such theories do. But the trope theorist has no need for such a posit and may stipulate that all known tropes exist at a location.

Given that the sufficiency condition for supervenience is not strong enough to give us an explanation of location-properties but that location-properties are possessed by other properties, we may need a stronger necessity condition. That would make a locationproperty a relation, but an odd one. As each object has its location merely by virtue of its existence, it is an internal relation. And as the only relatum for the location-property is the property or object which holds the relation that it does, the location-property is a reflexive relation. So on this view, location-properties are internal, reflexive relations of the things they relate. 
An objection here exists in the case of an area or location without any objects in it. This would be something such as an empty region of space-time. If area-properties are only to be had in supervenience to some other property and location-properties are only to be had in virtue of internal, one-place relations that other properties possess, where is the room for empty regions of spacetime? There are a few things that can be said to this objection. The first is that there may not be any such regions, as a contingent fact. Empirical science's investigations into the nature of physics have already revealed virtual particles and it is not implausible that at some future point, science will discover some object that occupies or is responsible for so-called 'empty spaces.' It may even turn out that space requires some underlying object or properties, and so empty regions are physically impossible. This response to the objection is purely speculative, however.

A better response comes in the form of a dilemma for one wishing to admit such regions into the ontology as a problem for constitutive trope theory. There are two possible routes one might take for discussing an empty region of space-time. The first is that such empty regions only possess spatio-temporal properties such as a shape, volume, and duration. If so, these properties are either foundational or emergent. If they are foundational, there is no problem for the theory as then empty regions of space-time are a sort of object, consisting of tropes of shape, volume, and duration. If these properties are emergent, there is similarly no problem as the spatio-temporal properties supervene and the region is also, then, an object. But one might argue that the regions are truly empty and devoid of any properties whatsoever. In this case, the regions are mere absences, which we comfortably eliminated from our ontology earlier. As such there are no 'objects' that are themselves merely empty space. 


\section{$\underline{\text { 3.5.3 Constructive Properties }}$}

I'll now turn our attention to the interesting case of constructive properties. A constructive property is the property of being constituted in a particular manner, such as the property of 'being made out of wood.' As any non-foundational trope on this theory exists entirely due to the relations of the constitutive parts that are sufficient for the existence of the trope, what should we make of properties such as 'being made of wood'? In other words, while a table's being made of wood may be responsible for some of its other properties, should the property of 'being made out of wood' be a true trope among the properties in its bundle?

There are two reasons to admit such properties into our ontology as tropes. First is a concern for simplicity. Constructive properties feature prominently in this ontology, as most of the tropes the ontology admits will turn out to be grounded in some such construction. If constructive properties play a prominent role in our ontology then we cannot simply reduce or eliminate them as we could historical properties or negative properties. If we do not admit constructive properties as tropes but do grant them ontological status, then we have increased the number of kinds of things our ontology admits, which would leave this theory open to the same objections that the trope theorist typically levels against the realist about universals. The second reason to admit constructive properties is that they feature prominently in scientific explanations. Facts about a molecule of water may require reference to the relationship between the hydrogen and oxygen atoms that are a part of its construction. If one of our goals is to provide a 
metaphysical grounding for such explanations, allowing the constructive trope may help in this.

There is one reason to be concerned about constructive properties as tropes. Consider an object X which has three tropes, $\mathrm{A}, \mathrm{B}$, and $\mathrm{C}$. A, B, and C are grounded in the relations (which we will call $\mathrm{R}_{\mathrm{P}}$ ) between the constitutive parts of $\mathrm{X}$. If $\mathrm{R}_{\mathrm{P}}$ is a series of tropes, then X actually has more than 3 tropes: A, B, C, and the tropes of Rp. So far, so good. But remember that what it is for an object to exist, on our view, is to be a bundle of compresent tropes. If $\mathrm{R}_{\mathrm{P}}$ is a series of tropes, then there will be an $\mathrm{R}_{\mathrm{p}}$ for any object that exists. While saying that there are relations between objects may not inflate one's ontology, saying that every object is just a collection of tropes and that each relation is itself a trope, we inflate the number of items in our ontology substantially, inviting other problems in as well. To put the problem plainly, we may want to admit a molecule of water into our ontology and we may want to admit 'being made of this hydrogen atom and those oxygen atoms' into our ontology, but objects such as 'my left shoe, the top of the Eiffel tower, and the moon' are unpalatable additions and it would be a negative consequence of the theory if we had to allow that to be an object merely because there was some relation between its supposed 'parts.'

A tempting answer is something like the following: that it takes more than one property to have a bundle. As such, merely having a single property does not make each relation into its own object. But this is insufficient as a response, as in real-world cases two objects will usually share many relations. They may have spatial, temporal, gravitational, electromagnetic, and other relations. In our earlier example, there may be a number of relations between 'my left shoe, the top of the Eiffel tower, and the moon,' not only one. If 
this is so, it would not eliminate such an object from our ontology. It is unlikely we can explain away all of these cases and still leave an account that can adequately accomplish all of our desiderata. Even if we remove negative properties and historical properties here, we are still left with a large, perhaps infinite, number of extra objects in our ontology, many of which are unpalatable. And if those relations can be said to have relations to each other, we invite Bradley's regress and other problems.

An answer is forthcoming, however, if we ask what tropes there are that arise from the series of Rp relations of these illegitimate objects. And the answer can only be that they have no properties aside from their constructive properties. But what makes RP a constructive property rather than simply a relation in our core case is that it results in the existence of new, emergent properties. A molecule of water possesses properties that its constituent atoms alone do not, and the atoms of that molecule possess properties that their subatomic constituents alone do not. For illegitimate objects, Rp does not. As the relation between objects is not itself an emergent property of the relation between those objects but is identical to it, we do not need to accept objects into our ontology if the supposed parts of the object do not result in any emergent trope coming into existence.

\subsubsection{Foundational Tropes}

Finally, we come to what may be the biggest problem for this theory: its treatment, or lack thereof, regarding foundational tropes. Foundational tropes are those tropes which do not depend on anything else, including other tropes, for their existence. While tropes do not, as a kind, depend on anything else for their existence on this account, many if not most individual tropes depend on some other trope for their existence. Modern science does not 
currently make claims of having found such properties: even fundamental physicists posit the existence of layers of properties below the ones they theorize about. Foundational tropes, by contrast, do not depend on any other object for their existence. This is what it is to be a foundational trope.

So far, foundational tropes have been mentioned, but no account of them has been given. One might reasonably present the following sort of criticism to this theory. The theory explains relations and objects in terms of tropes, and it explains emergent tropes in terms of constitutive parts, which are themselves objects, which are themselves composed of emergent tropes. So one may conclude that the theory does not explain anything at all, but instead keeps 'kicking the can down the road.' But the only account I will provide of foundational trope is a definitional one. A foundational trope is a genuine property that does not depend on any other property for its existence. This may seem unsatisfactory, since I am not even committing to the existence of foundational tropes. But there are several good reasons not to do so, as I will explain.

First, there is the possibility of atomless gunk, which has recently been defended in philosophy. ${ }^{138}$ While the typical defenses of 'atomless gunk' come from a mereology that is absent here, that does not mean that atomless gunk is incompatible with it. Personally, I find the idea that the objects of the universe are made of atomless gunk repugnant. However, the fact that this theory works whether or not the universe is composed of atomless gunk is a strength of the theory. If there are theoretical reasons to think that the universe does not divide down into atomless gunk, that eventually the question of "what

\footnotetext{
${ }^{138}$ Dean Zimmerman, "Could Extended Objects Be Made Out of Simple Parts?: An Argument for Atomless Gunk," Philosophy and Phenomenological Research 56, No. 1, (1996).
} 
composes this?" ends with a satisfactory answer, then all the better. But that this theory does not rule on the case of atomless gunk is both a strength and a limitation to the theory. It is notable that if the universe is composed of atomless gunk then this account is complete. For every level is explained in terms of emergent tropes and constitutive objects and there is nothing more to be said. Despite what it would mean for my theory, I still find atomless gunk repugnant, but the theory works whether or not the universe has foundational tropes or not.

Second, there is a matter of what I like to call 'philosophical humility' to consider. To put it simply: I do not know what the most basic properties of the universe are like. I simply have no knowledge of them. As far as I can tell, modern scientists are still speculating about what is below the quantum level, though it appears that even quantum objects may have constitutive parts. And there are reasons to suspect that whatever is on that level will defy our expectations about objects. We saw that in the case of quantum physics itself. For instance, prior to investigations into quantum objects, few philosophers took probabilistic laws seriously in investigations of scientific laws. Now an account of scientific laws is not complete without some talk about fundamentally probabilistic laws. Other ordinary parts of quantum theory such as quantum superposition and quantum entanglement have also informed philosophy and resulted in new problems for philosophers to consider in metaphysics. So when one thinks about what foundational tropes might look like, one needs to at least entertain the notion that they may be wholly unexpected and unlike anything one has thought of. If one has no knowledge of a thing and some reason to expect that the object may defy expectations in unexpected ways, then I think it is irresponsible for one to theorize about it. So as I have no knowledge of foundational tropes, theorizing about 
them is an activity that I take to be irresponsible. Calling whatever is most foundational 'tropes' may even turn out to be a mistake: what is occurring at the most basic level may be so wholly alien that I have no words for it. Whether or not this is true, I cannot say, but I have to acknowledge the possibility. As such, I will wait for the scientist to tell me which objects are foundational, with some reason to think that the level they point to is the foundational one, before I even begin to theorize about the metaphysics implied by such a discovery.

One may push the objection harder at this point. Given my position on what I take to be philosophical humility, one may argue that I am incapable even in principle of giving an account of foundational tropes. The account of emergent tropes is that they are grounded in parts which are sufficient for their existence. Yet there can be no such account for foundational tropes: if they exist, it cannot be due to some set of parts upon which they rely. Thus, there cannot be some set of objects or tropes upon which foundational tropes are grounded. Yet, this theory does not admit of anything else which might ground them. As a result, there appears to be no way of accounting for foundational tropes for constitutive trope theory. As all emergent tropes are grounded in yet-more foundational tropes, at least indirectly, this puts the whole theory in jeopardy.

Here I can only acknowledge the problem: in Chapter 5 when I discuss problems for the theory, I will theorize about the various options which are present and consider what accounts of foundational tropes are most damaging for the constitutive trope theorist. What I will say is that no matter what the account of foundational tropes is, it does not bring that baggage over to the rest of the theory. This is because the constitutive parts of a trope are a sufficient condition for the existence of trope which relies on them, not a necessary 
condition. To see why, consider two possible worlds, $\mathrm{w}_{1}$ and $\mathrm{w}_{2}$, both of which operate under the metaphysics of constitutive trope theory as laid out so far. Call the foundational tropes of $\mathrm{w}_{1} \mathrm{~F}_{1}$ and the foundational tropes of $\mathrm{w}_{2} \mathrm{~F}_{2}$, and the tropes which depend only on those foundational tropes $E_{1}$ and $E_{2}$, respectively. For the sake of simplicity, let's assume that all emergent tropes at $w_{1}$ and $w_{2}$ are either dependent on foundational tropes or emergent tropes, but given the physics of $\mathrm{w}_{1}$ and $\mathrm{w}_{2}$, neither world has any tropes that depend on both foundational and emergent tropes. Further, let's assume that $\mathrm{E}_{1}$ and $\mathrm{E}_{2}$ are indiscernible: the only differences between the emergent tropes at each world is that $\mathrm{E}_{1}$ is at $\mathrm{W}_{1}$ and $\mathrm{E}_{2}$ is at $\mathrm{W}_{2}$, and that $\mathrm{F}_{1}$ is sufficient for $\mathrm{E}_{1}$ and that $\mathrm{F}_{2}$ is sufficient for $\mathrm{E}_{2}$. Further, let's assume that $F_{1}$ and $F_{2}$ are incompatible: it is impossible for a world to both have $F_{1}$ type properties and $\mathrm{F}_{2}$-type properties. We might even assume they are substantially metaphysically different, provided that they are still sufficient for the emergence of $\mathrm{E}_{1}$ and $E_{2}$, respectively. Could such worlds exist? Yes, because $F_{1}$ and $F_{2}$ are each only sufficient conditions for $\mathrm{E}_{1}$ and $\mathrm{E}_{2}$. While $\mathrm{F}_{1}$ is sufficient for the $\mathrm{E}_{1}$ tropes at $\mathrm{w}_{1}$, the $\mathrm{E}_{1}$ tropes would be different only in their constructive properties if they were instead grounded in $\mathrm{F}_{2}$-type tropes. Scientists and philosophers at $\mathrm{w}_{1}$ and $\mathrm{w}_{2}$ could each successfully examine the E-type tropes of their world without knowing whether they even had $\mathrm{F}_{1}$ or $\mathrm{F}_{2}$ properties grounding them, regardless of the ontological baggage of those foundational tropes. And this is part of the power of constitutive trope theory: we may still theorize about the higher-level properties and their relations even if we do not yet understand the more foundational level. So while constitutive trope theory does not provide an account for the most foundational properties, this problem does not impact any other part of the account. 
Constitutive trope theory can handle all non-foundational properties and if that is all that it can do, then I take that to be an acceptable limitation of the theory. 


\section{Chapter 4: Scientific Laws and Constitutive Trope Theory}

The account of tropes provided earlier I will now refer to as Constitutive Trope Theory. Now that we have this theory to reference, we can attempt to design a theory of laws of nature that falls out of it without letting it collapse into either a realist theory by sneaking universals in through the back door or a nominalist theory which the trope theorist should have some reasons to reject. I will begin with a discussion of deterministic laws and from there move to probabilistic laws, in a manner consistent with other investigations into the nature of scientific laws. While probabilistic laws will provide us with an account which works in the case of deterministic laws, deterministic laws are easier to understand and so will provide the first steps toward our theory.

When scientists believe they have discovered a law, the law-statement then uttered is purported to be about the world in a particular sort of way. Law-statements make a claim about the objects in the world that can then be used to describe and predict the behavior of

those objects in a variety of circumstances. But a law-statement is not itself a law of nature. The law of nature is the ontological ground and truthmaker for the law-statement, but is not itself a mere statement. This distinction will be used throughout this chapter as we need to distinguish the utterances of scientists who are talking about laws of nature from the laws themselves.

\subsection{Deterministic Laws}

On first glance, much of the scientific literature focuses on laws that are deterministic in nature. A deterministic law is one where, ceteris paribus, the law applies in 
all instances of the phenomenon in question, and where the properties described by the law are the ones relevant to the phenomenon. As law-statements describe the behavior of objects, we may put this another way: that for any law-governed event, if that law is a deterministic law, then any variation of the behavior of the object is dependent on the properties of the object. Take Newton's Law of Motion, which says that the force on an object is equal to the product of its mass and its acceleration. For this law to be true, then, is for it to be the case that any change in the force on an object requires a change to its mass or acceleration in equal measure, and that as mass or acceleration increases, the force exerted upon an object increases.

The problem for a trope theorist, as discussed earlier, is that a law cannot be a multi-present entity. That is to say, it cannot be a universal. For a law to exist, it must exist as an entity or set of entities. So if tropes are to be the only sort of fundamental entity in our ontology, then laws must either be tropes themselves, be fully dependent on tropes, or be non-entities. I have addressed some of the problems that come out of the first option in discussing Campbell's account of tropes, and the third would violate one of our desiderata. So I will be examining the second option.

\subsubsection{Dependent Laws}

To establish that deterministic laws are completely ontologically dependent on the tropes which apply to them, we will begin with the following principle: that any change in an object requires a change in one or more of its tropes or in the relations between tropes which comprise it. That is, for some facet of an object to change, it must gain tropes, lose tropes, or both. A defense of this principle is relatively simple. As objects are themselves 
just a bundle of tropes which are dependent on a collection of constitutive parts in a particular relation, there is nothing aside from those tropes or the constitutive parts that can amount to a change. There is simply no other entity for a change to take place in. ${ }^{139}$ Now we will consider a claim about deterministic law-statements defended above: that any variation in the behavior of an object is dependent on the properties that object has. To put this in terms of our account of tropes, if there is a law regarding the relationship between two sets of properties, $\mathrm{H}$ and G, in a given object, then any variation in one implies a variation in the other. If an object with a mass of 60 grams and an acceleration of 2 meters per second per second had its acceleration increased to 4 meters per second per second, then the force on it would double along with its acceleration provided that its mass remains the same.

Of course, even if they inhere in the same object at different times, a $2 \mathrm{mps}^{\wedge} 2$ acceleration-trope and a $4 \mathrm{mps}^{\wedge} 2$ acceleration-trope are two different tropes. Our account of change in an object was just that of tropes being replaced because of changes in the relations of their constituent tropes. Law-statements, then, are not statements about tropes but are statements about sorts of tropes. We might then think that laws are a relation between sorts of tropes. This would be straightforward, as Newton's Law of Motion seems to be about force, mass, and acceleration, rather than particular force, mass, and acceleration tropes. But on our earlier account, however, sorts of tropes are not entities. Rather, two tropes are of a kind when they share a determinable. Two acceleration-tropes

\footnotetext{
139 I will be focusing on changes to tropes, rather than aggregates, as explaining aggregates involves a variation on this account that while essentially the same account becomes more complicated for the number of tropes and levels that become involved in such an account. So heretoafter in this discussion of laws of nature, all properties will be treated as tropes for the sake of demonstration.
} 
are 'of a kind' but not equivalent differ from each other in terms of their location and in terms of specificity but are still more alike than either is to a mass trope or a force trope.

Recall that for all emergent tropes, their existence and nature is an emergent property of lower level objects and the relations between them. This means that changes to non-fundamental tropes are due to changes in the lower-level objects from which they emerge. For an object $\mathrm{P}$ to change from having trope $\mathrm{a}$ to trope $\mathrm{b}$ is for the relations between the constitutive parts to change in some way such that $R_{a}\left(p_{a}\right)$ is sufficient for a and $\mathrm{Rb}(\mathrm{pb})$ is sufficient for $b$, noting that $\mathrm{R}_{\mathrm{a}}$ and $\mathrm{Rb}$ may or may not include all of the same members but that there is a spatiotemporal continuity between $R_{a}$ and $R_{b}$ such that $R_{a}$ is prior to $\mathrm{Rb}_{\mathrm{b}}{ }^{140} \mathrm{Law}$-statements are then statements about how changing the conditions that allow for one trope will affect other tropes in the bundle. If one changes the force upon an object, one must change its mass or acceleration in equal measure.

This forms the basis of the conditions necessary for a deterministic law. First, that for two tropes, A and B, the constitutive parts present be sufficient for these tropes to exist. Second, that any variation in those parts sufficient to replace A with $A^{*}$ will also replace B with $\mathrm{B}^{*}$. We can model these changes logically, as shown below. Of note, in the below model " $\supset$ " is material conditional, defined as $\mathrm{p} \supset \mathrm{q}=\mathrm{df} \sim \mathrm{p} \vee \mathrm{q}$. In contrast, " $\rightarrow$ " indicates a change from one thing to another over time.

1. $R_{1}(p)$ occurs $\supset$ A occurs

2. $\mathrm{R}_{1}(\mathrm{p})$ occurs $\supset \mathrm{B}$ occurs

\footnotetext{
140 Given our earlier examination of Newton's Law of Motion, it is important to note that for most objects, a mass is an aggregate rather than a true trope. However, it may still feature in laws as the mass of each individual particle is a part of the whole. As such, while most objects have mass-properties rather than masstropes, we may still talk about them in a lawlike fashion.
} 
3. $\mathrm{R}_{2}(\mathrm{p})$ occurs $\supset \mathrm{A}^{*}$ occurs

4. $\left(A \rightarrow A^{*}\right) \supset\left(B \rightarrow B^{*}\right)$

5. $\left\{\mathrm{R}_{1}(\mathrm{p}) \rightarrow \mathrm{R}_{2}(\mathrm{p})\right\} \supset\left\{\mathrm{A} \rightarrow \mathrm{A}^{*}\right\}$

6. $\left\{\mathrm{R}_{1}(\mathrm{p}) \rightarrow \mathrm{R}_{2}(\mathrm{p})\right\} \supset\left\{\mathrm{B} \rightarrow \mathrm{B}^{*}\right\}$

Lines 1-3 give us the constitutive property to trope relation, while 4 gives us a lawful relation between A-properties and B-properties. From this, we can trivially derive that any change that causes A to be replaced with $\mathrm{A}^{*}$ will also cause B to be replaced with $\mathrm{B}^{*}$.

This by itself is not enough to get us a law of nature, but it gets us there if we utilize the indiscernibility criteria from earlier. Recall that for two properties to be indiscernible is for them to have no differences save for those of which constitutive parts instantiate them. As the above lawful relation between properties is not a difference in instantiation, it will not be different between indiscernible properties. And as the above formulas are not specific with respect to the property A or to any property B, indiscernibility then allows for our rule to extend between multiple objects and their properties provided that the properties in the antecedent and consequent are each themselves of a kind.

This is then the account of what it is for something to be a deterministic law. A deterministic law relates two or more properties in such a way that any change in the constitutive parts resulting in a change to one of the properties determines changes in other properties governed by the law. For indiscernible properties, these laws will then hold between tropes given that indiscernible tropes are qualitatively identical, allowing for descriptive and predictive statements about the lawful relations regarding one property to be said of the descriptive and predictive statements about the lawful relations for the other. 


\subsubsection{Problems for Dependent Laws}

Immediately, there are a few concerns that need to be addressed. The first is a concern that these laws will lack the predictive power of laws on other accounts with respect to unexamined or uninstantiated properties. Second is that laws here will lack a necessity granted to laws on other accounts. I will address each of these in turn.

Let's consider the criticism that laws of this nature explained this way will lack predictive power. There are two versions of this problem, one with respect to scientific practice and the other with respect to metaphysics. With respect to scientific practice, the problem is easier to understand and answer, so I will start there. Take our earlier case of the law " $F=$ ma." Imagine there is some combination of mass and acceleration trope that has yet to be observed. It need not be anything too exotic: it may simply be that no one has ever actually done a measurement of force where a $427 \mathrm{~kg}$ weight was accelerated to 3.64 mps $^{2}{ }^{141}$ We have a law-statement, however, that tells us what the answer should be. There will in fact be an infinite number of such untested combinations. If this is so, then how on this account is the scientist justified in believing " $\mathrm{F}=$ ma" in general when there are an infinite number of scenarios that have never been tested and each test only shows a single dependence between a given mass, acceleration, and force? In other words, how is it that science is justified in making general claims about laws and tropes of a kind when there are in fact no 'universal' properties to be related in the theory?

There are, I think, two answers to this problem. The first is based in scientific practice. While law-statements and theories reference kinds rather than particular

\footnotetext{
141 There will be some masses and accelerations for which this is true, not because of any lack of ability to do so, but merely because we do not set out to measure every such combination in normal scientific practice.
} 
properties, the confirmation of such a law is done through specific instances. In fact, proposed law-statements are tested quite thoroughly before they are adopted by the scientific canon and it is not mere repetition that is required for a law to be adopted, but that the law is tested in a variety of circumstances. " $\mathrm{F}=\mathrm{ma}$ " as a law-statement would not be accepted if the tested mass was always $1 \mathrm{~kg}$ and the acceleration was always $1 \mathrm{mps}^{2}$, even if that test were repeated millions of times with no contradictory result. So in fact, lawstatements are tested against a wide variety of instances before their acceptance. There may always be untested instances, but the generality of law-statements provides the theoretical structure to test any suspected exception to the rule. Further, this process is encouraged by scientific practice: testing instances that one thinks might violate a lawstatement is good scientific procedure.

A further response lies in the relationship of tropes to their constitutive parts. When sorting out the metaphysics, calling the relationship between tropes and their constitutive parts a sufficiency relation is enough for our purposes. But for a given case in scientific practice it is not. A scientist wants to know why a collection hydrogen and oxygen when combined with a certain kind of bond such as to form water molecules freezes at a particular temperature. And in cases where tropes emerge from constitutive parts they may examine what, precisely, it is about the constitutive parts that is sufficient for the existence of the trope under examination, as those parts will also have tropes which operate lawfully. This more precise examination may reveal how these particular constitutive parts lead to this particular trope, revealing a relationship that implies information about unexamined tropes. For instance, if we examine a carbon atom with six protons and five electrons and determine that it has a positive charge, and find in other 
cases that when the atom has a number of protons greater than the number of electrons that it has a positive charge, we may deduce that even in as-yet undiscovered atoms, the presence of more protons than electrons will result in a positive charge. If one discovers how a particular combination of parts is sufficient for a particular trope, that knowledge may imply that if there were a different relationship between the object's parts another trope of the same kind would be present, a supposition which may then itself be tested.

A related worry of ontology is present in the case of uninstantiated properties. Take the case where the case of an uninstantiated property $\mathrm{p}^{*}$ which would be a trope if it were instantiated, and where such a p*-trope would be a p-kind trope if it were instantiated. Say that p-kind tropes exhibit a lawful relation wherein they produce q-kind tropes when in some scenario $\mathrm{S}$, and that given the relationship between $\mathrm{p}$-kind tropes and q-kind tropes in $S$, we would expect that if a $\mathrm{p}^{*}$-trope did exist in $\mathrm{S}$ it would produce trope $\mathrm{q}^{*} .{ }^{142}$ This may be taken to be a stronger case than the epistemic case counted earlier or a mere case of a negative property, because in all other observed cases there is a relationship between pkind tropes and q-kind tropes such that it seems we have very good reasons to predict what a $\mathrm{p}^{*}$ trope will produce but no ontological ground for this prediction. In fact, until there is a $\mathrm{p}^{*}$-trope in $\mathrm{S}$, it's not clear how one is justified in predicting anything about what will happen as there is no state of the world that grounds the prediction. Recall that it is only by supposition that $\mathrm{p}^{*}$-tropes are of $\mathrm{p}$-kind: in this thought-experiment, there are no actual $\mathrm{p}^{*}$-tropes at the time of the supposition. We predict that $\mathrm{p}^{*}$ will result in $\mathrm{q}^{*}$ because of the past success of the general $p \rightarrow q$ hypothesis, but as we do not have universals or

\footnotetext{
${ }^{142} \mathrm{q}^{*}$ may be a kind of trope that exists, if there are other circumstances which could produce a $\mathrm{q}^{*}$ trope, or it may not exist. It does not particularly matter for this thought experiment.
} 
anything like them to be related, we cannot ground $\mathrm{p}^{*} \rightarrow \mathrm{q}^{*}$ ontologically. This may not be a trivial objection either. Consider the case of biological kinds. If biology is a science with laws and if this theory of laws is going to take such laws seriously, as I have argued it should, then there are a good many biological properties that do not and have not existed but which may exist. Take Kleiber's Law, which states that an animal's metabolic rate scales to the $3 / 4$ power of the animal's mass. Say that there is no animal that weighs precisely 2 tons. ${ }^{143}$ We would expect that despite this, Kleiber's Law will hold for an animal that does weigh 2 tons. Yet on this theory, while there may be an epistemic ground for that prediction in the past success of Kleiber's Law, there is no ontological ground for it. The law may fail at precisely 2 tons.

I think that in the end this is a strength of this account, rather than a weakness. If a law holds or does not hold, it is because of some relation between the properties in question. To continue using biological laws, take the case of homozygous mutants parenting a normal offspring as an exception to the laws of Mendelian Inheritance. Here we have a case of a law and its exception. Some philosophers may want to think of laws as exceptionless, but in practice many accepted scientific laws come with ceteris paribus clauses. Even physical laws, such as 'metal will expand when heated' need clauses about things such as outside pressure which would make such laws fail to be universally predictive. And while it may seem odd in the general case, we need to leave an ontological ground for there being cases where a law fails to hold for unexpected reasons. We might not always be able to predict these reasons in advance, but when we are operating at the

\footnotetext{
${ }^{143}$ Even if an animal has weighed precisely 2 tons, there exists a time in which animals have existed but no 2 ton animals, which may still be a problem for the theory.
} 
level of generality of metaphysics I think we need to leave some room for exceptions to laws that as of yet have no grounding, for it may be that the interaction between the properties relevant to the law comes out to some unexpected but no less explicable exception to an otherwise good law.

Finally, there is the question of necessity. Some philosophers defend a notion of laws as having a kind of necessity ${ }^{144}$, be it logical, metaphysical, or a sui generis form of necessity, that this account of laws lacks. This line of thought goes as follows: if it is truly a law that "All p's are q's" or for our trope theory, that "All things with a p-kind property have a q-kind property," then unlike the accidental case where it just happens that each thing with a p-kind property has a q-kind property, there is something necessary about the fact that "All p's are q's." Defenders of this sort of necessity conclude that if we are to separate laws from accidents, then (in some sense) it must not only be the case that every p-kind thing is a q-kind thing, but that it could not be otherwise. ${ }^{145}$ One might think that this is not born out in this account, as the laws are dependent on the interactions of the tropes. Either the necessity of laws must be denied, or it will need to be shown that there is some form of necessity in this view of laws.

There is still a sort of necessity here, although it may not be as strong as one would like. The necessity comes out of the indiscernibility criterion: that for any two tropes that are indiscernible there is no difference between them except for difference in how they are instantiated. So, for any 2 indiscernible tropes, it is trivially the case that from observing

\footnotetext{
144 Examples include Armstrong, who was discussed in more detail in Chapter 2.

${ }^{145}$ A defender of necessity bringing up this sort of objection may have a more developed account of why laws of nature must have necessity built into them than is presented here, but this is enough to get the worry off the ground for our purposes.
} 
how the one trope is replaced in its bundle from a particular circumstance we can determine how an indiscernible trope would change in that circumstance, provided that the law in question is deterministic and our observation attaches itself to the appropriate phenomenon. As the two tropes are indistinguishable in virtue of their natures, this cannot be a mere accident. This gets us necessity for laws in the case where we have an indiscernible property with the indiscernible relevant relations to other properties.

What about cases where the properties differ by some degree? If the difference between two tropes is only a matter of degree, then any law-governed behavior for the object of the trope can (where the trope is relevant to the behavior) fall into one of three sets, all other things being equal. The first is that they exhibit indiscernible behaviors, the second is that they differ only proportionally to their difference in degree. This is because for them to differ only by degree, they must be similar in all respects other than degree. So they might exhibit the same behavior, as this would be consistent with their similarity. Or they might differ with respect to degree, as this would be consistent with the limitations of the difference between them. The third is that the change in degree changes the relations the trope has to other tropes in the bundle, causing a qualitative change after a certain point. For instance, when a solid changes to a liquid this is due to the relative strength of the kinetic energy of the molecules and the intermolecular attractive forces of those same molecules. If the kinetic energy is increased beyond a threshold determined by those attractive forces in a solid it will transform into a liquid because while the former trope's change may have been a matter of degree, its relative change to other relevant tropes was a matter of kind. This allows for three kinds of changes in the object: no change, change by degree, and change by kind, although the last of these is somewhat restricted. 
So while the law might not be directly governing the behavior of tropes, the indiscernibility of tropes combined with the law entails the sort of necessity we want. Further, as there are problems with making laws logically necessary this sort of entailed necessity is still empirical in character: laws of nature are proven to be such through a posterori investigation, and it is contrary to scientific practice to assume that such laws can be known a priori. ${ }^{146}$

\subsection{Probabilistic Laws}

Now that we have dealt with the case of deterministic laws, we can turn to the case of probabilistic laws. Unlike deterministic laws, the outcomes of probabilistic laws are not exceptionless. Instead, while the law is still taken to be exceptionless, the outcomes may be only probabilistic. There are two such categories of probabilistic laws: reducibly and irreducibly probabilistic laws.

Reducibly probabilistic laws are probabilistic only because of uncertainty in the problem. As an analogy, take the case of flipping a fair coin. The coin has a 50\% chance of coming up heads or tails. But we take it that this probability is not because of some irreducible fact about the coin. Rather, the $50 \%$ is a statement about our own ignorance. If we knew all of the facts: the angle and force of the flip, the air pressure and movement, the elasticity of the surface it lands on, etc. we would be able to predict the outcome of the flip with perfect accuracy. It is only our own ignorance that keeps us from knowing the answer.

\footnotetext{
146 The exact importance of empirical investigation to scientific practice, while of intense philosophical debate, is not what is being alluded to here. Rather, it is the mere fact that in practice, scientific laws are not accepted as such until they have gone under intense empirical scrutiny.
} 
Take the stylized facts promoted as a sort of laws by Nicholas Kaldor. ${ }^{147}$ One such stylized fact he proposed was "Output per capita grows over time." Even if true, this law will have numerous, unpredicted exceptions depending on the time scale we look at and it is in no way clear that these exceptions will be easily covered by non-arbitrary ceteris paribus clauses. Further, the behavior the law describes will be reducible to other properties rather than be a matter of the relations between genuine tropes. As such, this law-statement is a good candidate for a reducibly probabilistic law.

In contrast, an irreducibly probabilistic law's probabilities are not merely a matter of ignorance. Instead, while irreducibly probabilistic laws will show a regularity, the regularity they show will not predict one outcome but will instead indicate the chance that any given potential outcome occurs: not as a matter of ignorance but as a base matter of the phenomenon in question. Take the Schrödinger equation, which can predict the probability distribution for potential outcomes of the measurement of the momentum and position of a particle but not the precise result of any such measurement. On most interpretations of quantum mechanics this is not because of some unknown factors as was the case with stylized facts. Instead, the nature of the phenomenon is inherently probabilistic: one cannot predict in advance what the measurement of a phenomenon governed by the Schrödinger equation will yield because there is no answer in advance of one's measurement and the collapse of the wave function. The system is fundamentally probabilistic.

${ }^{147}$ Nicholas Kaldor, “A Model of Economic Growth," The Economic Journal Vol 67, Issue 268: 1 (Dec 1957): 591-624. 


\subsubsection{Irreducibly Probabilistic Laws}

We will begin with the case of irreducibly probabilistic laws, as an examination of these will fundamentally change what needs to be said about laws and will further inform our trope theory. In the case of deterministic laws the relation between the properties governed by the law is one where a change in one property always produces a change in the other in virtue of the constitutive parts responsible for the properties in question. However, in the case of irreducibly probabilistic laws it is not the case that a particular change is always produced. Instead, a particular outcome only has a propensity to occur when its behavior is governed by an irreducibly probabilistic law.

This may represent the simplest solution to the problem of irreducibly probabilistic laws: to simply change the talk from how properties relate to talk of a propensity of properties to relate. In other words, to say that there is a law is to say that there are two or more properties of an object such that changing the constitutive parts in a way that changes one or more of said properties creates a propensity to change other properties governed by the law. Changing the momentum of a particle governed by the Schrödinger equation creates a propensity to change its position, not a guarantee of a particular change in position. In the case of deterministic phenomena, this propensity is simply maximally efficacious: it occurs in all cases, all other things being equal. Probabilistic laws are only different in that this propensity is not as strong or is divided between multiple competing options.

This creates two problems for our theory, one with respect to what it is for a trope to have a propensity, and another with the indiscernibility criterion. The former is that now tropes must be such that they are the sort of thing that can have a propensity and we must 
define what it is for a trope to be such. The latter is a problem as there appears to be a way to distinguish between maximally similar tropes if those tropes have probabilistic laws governing the phenomena to which they take part: namely, in terms of the consequences of their causal relations.

\subsubsection{Dispositional Tropes}

So what would it look like for tropes to be the sort of things to have propensities within them? Dispositional essentialism, as promoted by Martin ${ }^{148}$, Swoyer ${ }^{149}$, Ellis ${ }^{150}$, and Bird $^{151}$ may provide us with an answer. For the dispositional essentialist, a law of nature is to be grounded in 'powerful properties,' that is, properties which themselves are defined as having certain causal powers. On the classic Humean picture, properties do not have causal powers. As Hume himself said:

"What is our idea of necessity, when we say that two objects are necessarily connected together. Upon this head I repeat what I have often had occasion to observe, that as we have no idea, that is not derived from an impression, we must find some impression, that gives rise to this idea of necessity, if we assert we have really such an idea. In order to this I consider, in what objects necessity is commonly supposed to lie; and finding that it is always ascribed to causes and effects, I turn my eye to two objects supposed to be placed in that relation; and examine them in all the situations, of which they are susceptible. I immediately perceive, that they are contiguous in time and place, and that the object we call cause precedes the other we call effect. In no one instance can I go any farther, nor is it possible for me to discover any third relation between these objects." 152

148 C.B. Martin, “Dispositions and Conditionals," The Philosophical Quarterly 44, No. 174, (Jan 1994).

${ }^{149}$ Chris Swoyer, "The Nature of Natural Laws," Australasian Journal of Philosophy 60, No. 3, (1982).

150 Brian Ellis, Scientific Essentialism, (Cambridge, Cambridge University Press, 2007).

151 Alexander Bird, Nature's Metaphysics: Laws and Properties, (Oxford, Oxford University Press, 2009)

152 David Hume, A Treatise of Human Nature, HarperTorch, (2014). 
To say that one thing is a cause and another is an effect is only to say that they are contiguous in time and place and that the cause precedes the effect: there is no power that the cause possesses to produce the effect. This is precisely what the dispositional essentialist denies. According to the dispositional essentialist, what it is for a physical property to exist is precisely for it to have certain causal powers. A dispositional essentialist invites the following sort of thought experiment: imagine a property that is like the property of having a negative charge in all respects but one: having this property confers an attraction to things that have like properties (that is, other 'negative charges') rather than the opposite. The dispositional essentialist wants to say that this property would not be a negative charge with some different entity, a law, governing it. Instead, this property would not be a negative charge at all: it is distinct from negative charges in that it confers a different causal disposition.

There are three questions to be answered if this account is going to take on dispositional essentialism. First, are tropes on this account dispositional? Second, if tropes are dispositional, are these dispositions essential to the nature of the trope? Third, are all tropes dispositional in nature? One might think that an affirmative answer to the second question implies an affirmative answer to the third, as most accounts do. But as has been pointed out by Jaag ${ }^{153}$ as well as Hendry and Rowbottom, ${ }^{154}$ we can separate out these questions. One could hold that some tropes are dispositional and others are not, or one

\footnotetext{
${ }^{153}$ Siegfried Jaag, "Dispositional essentialism and the grounding of natural modality," Philosopher's Imprint (2014).

154 Robin Hendry and Darrell Rowbottom, "Dispositional Essentialism and the Grounding of Laws," Analysis 69, No. 4, (Oct 2009).
} 
could hold that, while each trope carries a disposition, these dispositions are not always essential to the nature of the trope.

First we must ask if tropes on our account are dispositional, and the answer appears to be a resounding yes. We already have spoken of tropes as having a propensity to relate to other properties. To have a propensity to have particular relations with other properties is to have a disposition to behave in a certain manner in the presence of those properties, as if two properties change with respect to one another, they trivially do so via some relation between each other. Further, in discussing deterministic laws we've already committed to those laws being grounded in the behavior of properties rather than in some outside entity that is the 'law of nature.' As such, it appears we are already committed to tropes as dispositional, at least in the case where there are laws regarding them.

In answering the second question of whether or not dispositions are essential to the nature of a trope, we must break this into two questions. The first is if tropes have their dispositions necessarily. The second is if a trope is identical to its dispositions. While dispositional essentialists generally affirm both theses ${ }^{155}$ we should consider them separately here. The first question can be examined with the following: could a trope have a different disposition than the one it has and still be the same trope? If so, then it cannot have that disposition necessarily. If not, then it is impossible to separate the trope from the disposition and we have our necessity.

The question under examination may at first appear intractable, as answering it would require answering what it is that makes one trope 'the same' trope as itself. Such questions are notoriously difficult. However, we can answer the question if we turn to

${ }^{155}$ Choi and Fara (2012) define dispositional essentialism in terms of accepting both of these theses. 
maximally similar tropes. Recall that any two tropes are maximally similar if the only difference between them is what instantiates them. As the question of what dispositions a trope has is not a question of how they are instantiated, we may examine two maximally similar tropes as if they are identical for the purpose of this question. So we can ask: is it possible to have two maximally similar tropes with different dispositions? And then the question becomes easy. If two otherwise maximally similar tropes had different dispositions, they would be distinguishable in a way other than their instantiation. As such, it seems that changing the disposition of a trope would make it a different trope than the one that it is.

An objection to this presents itself, however, in that we may think the above test illegitimate. Some attempting to deny that tropes have their dispositions necessarily may think that changing the disposition of a single trope in our thought experiment is not how one would argue that tropes do not have their dispositions necessarily. Instead, they would argue that in such a thought experiment, all maximally similar tropes would need to be changed. I deny this, as tropes are particularized properties. Each trope is an independent entity which is not dependent on its 'kind' or some other taxonomic partition to be the sort of thing that it is. Changing the nature of one trope does not change anything essential to other tropes: it can at best change those tropes' relations to it. For instance, changing the nature of one trope may lead to other tropes no longer being maximally similar to it. Demanding that all maximally similar tropes be changed merely obfuscates the issue, as tropes each have their nature independent of others of their kind. If tropes do not have their dispositions necessarily, then we can talk of the same trope having a different disposition and this talk is legitimate. So one who holds to a trope ontology and thinks that 
tropes do not have their dispositions essentially is bound to accept all of the parts that lead to this conclusion.

As to the second question, of whether or not tropes are anything over and above their dispositions, we must again answer two questions. First is how we distinguish between a given disposition and a trope. Take the property of exerting a force as examined earlier. A force property has a lawful relationship to mass and acceleration in the same object, as described by Newton's Second Law. A force property also has a lawful relationship to other force properties it interacts with, as described by Newton's Third Law. These are different dispositions: there is no contradiction in Newton's second law being different without the third law being different and vice versa. Given that these dispositions are distinct we can ask if a trope is a disposition, if it is a cluster of dispositions, or if it is something over and above that 'links' the dispositions together?

This problem mirrors the problem between the bundle theory and the substratum theory of tropes and objects. And for similar reasons, I think we can rule out tropes as a substratum for dispositions. If we allow this, then our ontology has two sorts of things: dispositions and tropes. Further, tropes themselves become a sort of bare particular on this view and we are then required to ask what it is to be a 'dispositionless trope.' What is 'Force' over and above its disposition to behave in a certain way with respect to other properties? The only potential answer is that it is what unifies the dispositions. But this gets us into familiar regress problems for bare particulars: does the dispositionless trope have a disposition to unify the dispositions that are a party to it? If so, then it is not dispositionless. If not, then how can it perform the unification if it is not disposed to do so? 
As 'bare tropes' has the same problems as 'bare particulars,' I think they should be abandoned, largely for the same reasons.

Technically, this is enough of an answer to the second question to determine whether a trope theorist who accepts this account should be a dispositional essentialist, as there is nothing over and above the dispositions that makes a trope the sort of thing that it is. However, there is an important question of ontology that has been raised as well and I believe answering it is best. And that's the question of whether or not the true tropes just are the individual dispositions or whether tropes are bundles of dispositions? If the former, then perhaps we shouldn't talk of tropes at all: just dispositions.

However, I do not think we can eliminate talk of tropes this way or merely reduce tropes to bundles of dispositions. There are two reasons for this. The first is that dispositions are more tightly bound to each other than the tropes of an object are. Consider the case of a particle's tropes: mass, spin, charge, etc. As discussed earlier, some of these tropes may be accidental while others will be essential. However, tropes have no 'accidental dispositions.' As shown in the previous example, to change any disposition a trope possesses requires that we change the nature of the trope in question. We have examples such as a particle changing its velocity to consider for whether or not an object can change, but tropes do not change dispositions in this way. That is, every disposition a trope has, it has essentially. The trope is identical to the bundle of dispositions, but tropes are not as easily separated into their distinct dispositions as objects are into their distinct tropes. Further, there are no dispositions distinct from tropes. A trope might consist of a single disposition, but even if it does then that single disposition is itself a trope. Because of 
this, tropes are still the appropriate fundamental entity for our metaphysics, rather than dispositions. $^{156}$

Lastly, we need to ask if all tropes have dispositions: that is, if there are dispositionless tropes. I have already tipped my hand on this somewhat in my earlier examination of 'bare tropes' but there is an important distinction to be made between saying that a trope with dispositions is nothing over and above those dispositions and that all tropes have dispositions. In other words, it may be that if a trope has one or more dispositions that the trope is nothing over and above those dispositions and yet there may be tropes with no dispositions.

So do dispositionless tropes exist? At first glance, there is no a priori reason to rule them out but also no reason to include them. So it would be an empirical matter whether or not dispositionless tropes exist. But this creates a tension. Dispositionless tropes cannot be discovered via the senses, as in order to be sensed a property must have some disposition: to reflect or produce light, to produce or occlude sound, etc. This makes dispositionless tropes immune to empirical investigation. Even the light and sound produced must have dispositions or it cannot interact with our sensory organs: if light had no dispositions, we would never discover its existence. One exception might be the sensation itself: if the qualia that we experience are or are composed of tropes, perhaps we can know (some of) these qualia-tropes directly. But even qualia contain dispositions. In brief, one can either identify

\footnotetext{
${ }^{156}$ Note that this is a methodological point rather than an ontological point. I take it that talk of tropes is more useful than talk of dispositions in many cases. As all tropes have their dispositions essentially and there are no 'dispositionless tropes' in the ontology, we may also talk about dispositions apart from tropes. Tropes will turn out to be identical to their dispositions on this view, so neither is truly a more fundamental entity for our ontology than the other. But for methodological reasons, I will continue to ground the ontology in tropes rather than dispositions. Further, this will help the third part of our account.
} 
qualia as our sensory experiences or as momentary experiences. Our sensory experiences change as our sensory input changes: qualia must then be disposed to change as our inputs change. Our momentary experiences are different, rather than change, they exist for a moment before leaving us. But even these momentary experiences have dispositions: they affect our memory, for instance, and if they did not then we would not be able to recall them. But there may be one other candidate in this chain: the 'I' that has these experiences. Yet even this I is not dispositionless: if nothing else, it has the disposition to receive signals from the relevant sensory apparatus. So there is no point at which a dispositionless trope can be observed in the empirical chain.

This amounts to a weak denial of dispositionless tropes: it is not that there is some reason against admitting them, only that there is no reason to posit their existence. As we have affirmed the first two points in favor of dispositional essentialism but only weakly affirm the third, I will refer to our theory as promoting a pragmatic dispositional essentialism. As all known tropes are dispositional and as all dispositional tropes are nothing over and above their dispositions, the theory tentatively embraces a sort of dispositional essentialism with the caveat that dispositionless tropes are not strictly speaking ruled out, but rather there is no reason to posit them given this theory.

\subsubsection{Indiscernibility and Dispositional Probabilistic Tropes}

This leaves the problem of whether or not two maximally similar tropes can be distinguished on this theory if they possess equivalent fundamentally probabilistic dispositions but have a different history with respect to those dispositions being actualized. We may think that this presents no special issue for our theory, as in Chapter 3 we 
eliminated historical properties from our ontology. While this will go some way to eliminate the problem of discernibility among tropes with fundamentally probabilistic dispositions, it does not fully eliminate the problem. For even if we can eliminate historical properties from the ontology and thus eliminate worries about probabilistic dispositions causing otherwise maximally similar tropes to be distinguished on the basis of their history, we can still distinguish them in the moment of their disposition being actualized.

To see this, take two tropes, A and B. A and B are maximally similar and when exposed to a set of circumstances each has a disposition to change from $A$ to $\mathrm{A}^{*}$ and from $\mathrm{B}$ to $\mathrm{B}^{*}$, with $\mathrm{A}^{*}$ and $\mathrm{B}^{*}$ being maximally similar tropes. Further, this disposition is fundamentally probabilistic with a probability that is neither 0 nor 1 . At time $\mathrm{T}, \mathrm{A}$ and $\mathrm{B}$ are both exposed to the circumstances sufficient to actualize their disposition. As it happens, A enters into the process to change into $A^{*}$ while $B$ does not enter into the process to change into $\mathrm{B}^{*}$. At the time after $\mathrm{A}^{\prime}$ s disposition is actualized but before it transforms to $\mathrm{A}^{*},{ }^{157} \mathrm{~A}$ and B are distinguishable by A's disposition being actualized and B's not. Yet if A and B are maximally similar, then A and B should not be distinguishable.

To analyze this case, we need to recall that for all non-foundational tropes, there are some constitutive parts that are sufficient for their existence and that any change for the trope must be in terms of those parts. In other words, for A to change into $\mathrm{A}^{*}$, the relation between the parts $R_{A}(P)$ must become $R_{A^{*}}(P)$. So to say that $A$ is disposed to change into $A^{*}$ is only to say that the relation between the parts sufficient for its instantiation are changing. So it is not that $A$ is disposed to change into $A^{*}$, but rather than $R_{A}(P)$ is disposed to become $\mathrm{RA}^{*}(\mathrm{P})$ under some circumstances, and that $\mathrm{R}_{A}(\mathrm{P})$ is sufficient for $\mathrm{A}$ but not $\mathrm{A}^{*}$

157 Depending on the nature of the change, this might be instantaneous or a drawn out process. 
and that $\mathrm{R}_{\mathrm{A}^{*}}(\mathrm{P})$ is sufficient for $\mathrm{A}^{*}$ but not $\mathrm{A}$. But what if $\mathrm{A}$ is a foundational trope? Here I will have to continue to remain agnostic about the natures of foundational tropes, for reasons broadly outlined in Chapter 4.158

But this may not be the only sort of case we need to analyze. For it might be not that the trope's probabilistic nature alters the trope, but that it alters some other trope in its bundle. Take again two maximally similar tropes, A and B. Only this time, A is bundled with a trope $\mathrm{C}$ and $\mathrm{B}$ is bundled with a trope $\mathrm{D}$, with $\mathrm{C}$ and $\mathrm{D}$ also being maximally similar tropes. And there is a relation $\mathrm{R}_{(\mathrm{A}, \mathrm{C})}$ between $\mathrm{A}$ and $\mathrm{C}$ such that $\mathrm{A}$ has an irreducibly probabilistic disposition to change $\mathrm{C}$ to $\mathrm{C}^{*}$ when exposed to some set of circumstances. And there is a maximally similar relation $\mathrm{R}_{(\mathrm{B}, \mathrm{D})}$ to change $\mathrm{D}$ to $\mathrm{D}^{*}$, with $\mathrm{C}^{*}$ and $\mathrm{D}^{*}$ also being maximally similar. As it happens, A and B are both exposed to these circumstances at the same time, and while A's disposition actualizes and changes $\mathrm{C}$ to $\mathrm{C}^{*}, \mathrm{~B}^{\prime}$ s does not actualize with respect to D. While our earlier case handles the change from $C$ to $C^{*}$, this is distinct from A's disposition being actualized and B's not. Thus, they are distinguishable in that moment given A's actualized disposition despite being otherwise maximally similar. Further, as the disposition of A was irreducibly probabilistic, we cannot kick the can back to the relations between their parts being responsible for the difference between A and B here.

To account for this case, we need to ask what it is for A's disposition to be actualized while B's is not. As they both have the same disposition, what is supposed to be the distinction? It can only be that the relation between $R_{(A, C)}$ is such $A$ is causally responsible for $C$ changing to $C^{*}$ while $R_{(B, D)}$ is not. But this means that $A$ has a relation to which $B$ has no equivalent, namely, $\mathrm{R}_{\left(\mathrm{A}, \mathrm{C}^{*}\right)}$. That is, its relationship to the property $\mathrm{C}^{*}$. As there is no $\mathrm{D}^{*}$

\footnotetext{
${ }^{158} \mathrm{~A}$ further investigation of foundational tropes will occur in Chapter 5.
} 
for B to be in said relation to, no such relation exists. And we do not require that two otherwise maximally similar tropes share in all of the same external relations: two charge tropes may be maximally similar but share in all kinds of external relations to which the other is not a part. As the difference between A's disposition being actualized while B's is not is wholly cashed out by the presence of the external relation between A and $\mathrm{C}^{*}$, there is no reason to posit any internal difference between A and B. As such, there is no problem with A's disposition being actualized while B's is not.

\subsubsection{Reducibly Probabilistic Laws}

Now that we have discussed the solution to and problems with irreducibly probabilistic laws and some of the problems that arise from them, we may address the question of what it is for a law to be reducibly probabilistic. A reducibly probabilistic law is one where a property or object meets the following three criteria:

1) The property in question has a disposition in a given circumstance.

2) The disposition is probabilistic, which is to say that it does not always actualize in said circumstance.

3) That probability is not due to some facet of the property itself but is instead entirely due to the behavior of its parts.

Take Verdoorn's Law, an economic law which states that productivity generally grows proportionally to the square root of output in the long run. If we admit to such economic properties as 'productivity' and 'output' then the first criterion is met. This law is certainly probabilistic, meeting the second criterion. That leaves only the question of whether or not it meets the third criterion. 
This turns out to be a more problematic question than one might think, because we need to be able to distinguish whether or not a probabilistic disposition is due to a dispositional nature of the property or if it can be reduced to the nature of its parts. In this way, reducibly probabilistic dispositions are much like aggregates: they are not genuine dispositions but are instead descriptions of the aggregate behavior in the constitutive parts of the object. But explicating what it is that makes something a reducibly probabilistic laws as opposed to an irreducibly probabilistic law gives us the answer: irreducibly probabilistic laws regard the behavior of the property in question while reducibly probabilistic law regard the behavior of the parts. Given that a particular collection of relations between constitutive parts is only a sufficient condition for a given property and that properties are able to be multiply realized, this gives us at least a thought experiment we may use to discern the difference.

This thought experiment goes as follows: take a property P with a certain disposition $\mathrm{D}$ at a time $\mathrm{t}_{1}$. Imagine that $\mathrm{P}$ is instantiated by $\mathrm{R}_{\mathrm{p}}(\mathrm{p})$ at $\mathrm{t}_{1}, \mathrm{R}^{*}\left(\mathrm{p}^{*}\right)$ at $\mathrm{t}_{2}$, and that $t_{1}$ and $t_{2}$ overlap such that there is always some sufficient condition for P. If $D$ is an irreducibly probabilistic disposition of $\mathrm{P}$, then $\mathrm{D}$ cannot change merely because the constitutive parts of $\mathrm{P}$ have changed. If $\mathrm{D}$ is a reducibly probabilistic disposition of $\mathrm{P}$ at $\mathrm{t}_{1}$, then unless $\mathrm{R}_{\mathrm{p}} *\left(\mathrm{p}^{*}\right)$ also carries $\mathrm{D}$ then $\mathrm{D}$ does not survive the overlap. Thus, for a given disposition and property to which it attaches itself, if a change of the constitutive parts that is not sufficient to alter the property is sufficient to alter the disposition, then the disposition is a reducibly probabilistic disposition that in truth is attached to the 
constitutive parts with the property in question being at-best related to the disposition rather than indicative of it. ${ }^{159}$

\subsection{Dispositional Essentialism}

Dispositional essentialism is, by the above reasoning, a strong candidate for a trope theorist looking to explain laws of nature without resorting to multipresent entities. But before adopting such a position, we need to consider two different strategies for the trope theorist to use with respect to dispositional essentialism: dispositional monism, or a mixed view where tropes are both dispositional and categorical. Here it must be said that by accepting dispositional essentialism, a constitutive trope theory differs from other views on which properties are merely categorical. For the dispositional essentialist, tropes can themselves be the ground of modal truths. This is a significant bullet for the constitutive trope theorist to bite: a dispositional essentialist will have to defeat Humean intuitions that properties cannot themselves be the ground for modal truths about the world.

Putting that issue aside for the moment, a trope theorist who is a dispositional essentialist will need to decide between dispositional monism and a mixed view. If the former is the case, then either it will turn out that tropes are themselves just dispositions or that tropes reduce to dispositions, while if it is the latter then the trope theorist will need to explain how a trope can be both modal and non-modal in character. Bird notes that

\footnotetext{
159 Given this, whether or not Verdoorn's Law is actually reducibly or irreducibly probabilistic is left to empirical investigation. While it may seem a priori that a law of economics is more likely to be reducibly probabilistic while a law in a "more scientific" field such as quantum mechanics will have irreducibly probabilistic laws, this does not directly follow. As some interpretations of quantum mechanics hold that the laws of quantum mechanics are actually only reducibly probabilistic, it is at least conceivable for quantum mechanical laws to be reducibly probabilistic while economic laws are not.
} 
the primary decider of this debate may be how well one thinks dispositions handle structural properties. ${ }^{160}$ This is because structural properties, such as existing in certain spatial or temporal relations to other properties, are perhaps our best examples of properties where a disposition does not seem central to the property's nature. An object's being red, for instance, may be grounded in how the object is disposed to absorb or produce light. By contrast, a structural property such as being "three feet to the right of" may feature in dispositions but, Bird argues, does not itself appear at first glance to be inherently dispositional. I agree but think that this is due to a deeper question: are there any tropes whose essence is not exhausted by a full, complete listing of their dispositions? Mellor argues for the idea that a property's essence may be exhausted by its dispositions. In one case he uses the example of triangularity, saying that it has the following dispositional character: That $x$ is triangular entails that if someone were to count $x^{\prime}$ s corners correctly, then the result would be three. Barring doubts over whether this is as an ontological disposition, ${ }^{161}$ we need to ask whether or not this, along with whatever other dispositions a triangle may possess, exhaust its character. In other words, we must ask whether the entirety of a trope must either itself be a disposition or reduce to a set of dispositions.

I do not believe this is so. Structural properties may feature in dispositions: after all, it was shown in Chapter 3 that structural properties feature in the constitutive relations of emergent tropes. However, structural properties are not exhausted by these dispositions. Take Mellor's disposition of triangularity. Even if we take Mellor's candidate to be a part of triangularity's disposition and not due to some facet of counting or another analytic or

\footnotetext{
160 Bird, Nature's Metaphysics: 218.

${ }^{161}$ Prior (1982) brings up several problems with Mellor's version here.
} 
epistemic condition, we must ask if this, combined with a full set of dispositions of triangularity, exhaust its character. I do not think this is so. For one, Mellor's triangularity disposition seems to get the nomic grounding backwards: it is not the fact that 'if someone were to count $x^{\prime}$ s corners correctly, then the result would be three' that makes something triangular. ${ }^{162}$ Rather, it is that if something is triangular and someone were to count its corners correctly, that the result would be three. To say otherwise is to ground the nature of something's being triangular in the act of counting. I think this gets at a significant problem for dispositional monism. If all properties are merely dispositions or a collection of dispositions, then what it is for something to be the kind of thing that it is entirely dependent not only on other real properties but also in any possible property which may feature in a dispositional relation to it, which leads us down a dangerous regress.

To see this, assume the truth of dispositional monism and take a given property, $x$. If dispositional monism is correct, then $x^{\prime}$ s nature is exhausted by the sum of dispositions that, on this view, compose it. But this will include not only its disposition to behave when exposed to real properties, but to any possible property or collection of properties for which a stimulus-response manifestation may hold for that property. This will be true even if there are no other such properties. Take a world with only one electron. ${ }^{163}$ This electron's properties of mass and charge will cause it to have a certain dispositional character with respect to other objects with mass and charge, even though no such other objects exist at its world. Because if such objects were to exist and interact with the electron, the electron would act in certain ways. So possibilia necessarily feature in our

\footnotetext{
162 Mellor (1991) affirms this.

163 This may include whatever constitutive parts give rise to an electron, provided that it turns out that mass and charge are had non-derivatively for our example.
} 
ontology if dispositional monism holds: in fact, what it is for an object to be the kind of object it is not only depends on that object but on every other possible object to which it would react in a particular way given the right conditions, if that object were to be actual. Is this so for the mixed view? If the mixed view must also admit possibilia into its ontology in the same sort of strong sense used above, then it does no better than dispositional monism. There are a number of ways to cash out a mixed view of modal- and non-modal aspects of a property, and while some of them may still retain this problem others will not. One strong candidate is, I believe, the view that any genuine property has a core character of being which grounds its modal aspects. In this way, tropes are dispositional but are not themselves merely dispositions. Instead, the dispositions are grounded in the character of the thing to which they belong. This is the opposite of the sort of dependence that the dispositional monist draws: tropes are not dependent on or reducible to some set of dispositions, but rather what dispositions an object has depend on its tropes.

So what is the 'core character of being' which is itself dispositional? For emergent tropes, it is to be just a property that exists due to a sufficient set of relations between its constituent parts. Having an acidity of $13.995 \mathrm{pKa}$ is a property which exists when a configuration of one molecule of hydrogen and two molecules of oxygen with certain molecular bonds exists ${ }^{164}$. This acidity will imply certain dispositions, as will other tropes. That a given ' 13.995 pKa'-property exists is just for there to be conditions sufficient for its existence. The dispositions that result are then dependent on the nature of that property, as they are aspects of that property. But what about foundational tropes? As argued earlier,

\footnotetext{
164 There are, of course, other conditions sufficient for the existence of this property.
} 
I can say little about these: I do not know what they look like or what they will be. I have no direct experience with foundational tropes and I do not believe that science has yet found any good candidates for them. ${ }^{165}$ They might also not exist. As such, I believe any speculation as to the core character of their being would be premature at this time.

The above discussion is not wholly complete, but it is sufficient to indicate the sort of dispositional essentialism argued for here. Laws of nature are grounded in the fact that tropes are inherently dispositional, but tropes are not inherently reducible to dispositions, for their core character is not dispositional. Rather, the character of emergent tropes is grounded in the conditions sufficient for their existence. This character implies certain dispositions, which are then the ontological ground for laws of nature. This also answers Bird's challenge for a 'mixed' theory to account for how a trope can be both modal and nonmodal in character: while tropes are inherently dispositional, their core character is not. Their core character is decided by the constitutive parts that give rise to their existence, and this character will then imply certain dispositions which apply to the trope in question.

\subsubsection{Nomic Generalizations}

We've already showed, in dealing with probabilistic laws, how characterizing those as describing a disposition of the trope is useful. However, it is useful here to then discuss how dispositions can generally speaking be used to explain nomic generalizations. Humean expression of nomic generalizations take the form $\forall x(F x \supset G x)$ or "All F's are G's." As our account is modal, this will not express all that the constitutive trope theorist would want in a nomic generalization. The form is still useful, however, to describe how it is that

\footnotetext{
165 A discussion on why occurs in Chapter 3.
} 
dispositional essentialism differs from other accounts with respect to nomic generalizations. Putting aside the modal changes that will be required, for the dispositional essentialist, translating this Humean expression into talk of dispositions requires it be translated into stimuli and manifestations. This takes the form of $\forall x((F x \& S x) \supset M x)$ which translates more to, "If $\mathrm{x}$ is an $\mathrm{F}$ and is subject to this particular stimulus $\mathrm{S}$, it has a given manifestation M," or "All F's manifest M when subject to S."

This becomes more complicated when we include probabilistic laws, and more complicated yet when we introduce ceteris paribus laws. For the probabilistic law, the question becomes where best to formally indicate the probability. If it replaces the universal quantifier then this creates two problems. First, it looks like for any given trope this brings us back to a mere accidental generalization. There are, after all, a number of accurate accidental generalizations that can be made about how a trope might manifest after being exposed to some stimulus, even if there is no nomic relation between the manifestation and the stimulus. And there is a reason to think such accidental generalizations are not laws even when accurate.

Take the following sort of example: say that a given particle xa has a 50/50 chance to manifest behavior A or behavior B entirely on the basis of its having trope ta, when exposed to a stimulus $\mathrm{S}$, where $\mathrm{A}$ and $\mathrm{B}$ are exclusive and exhaustive manifestations of xa when exposed to S. Call all tropes indiscernible from ta t-tropes and all particles composed of tropes indiscernible from those in particle xa x-particles. Thus, all x-particles bear a ttrope. If we run a number of $\mathrm{x}$-particles through an experiment where each is exposed to $\mathrm{S}$, we may find that they all exhibit behavior A for purely probabilistic reasons, or we may 
find some ratio other than 50/50 for their manifestation. ${ }^{166}$ Nonetheless, we might have good theoretical reasons for believing that the disposition of $\mathrm{x}$-particles to manifest $\mathrm{A}$ is $50 \%$ and not some other probability. If the probability replaces the universal quantifier above, this creates two problems. First, it is unclear what there is to say about the nomic relation when we talk about individual tropes and their dispositions rather than groups of maximally similar tropes. Second, it's unclear why we should accept the theoretical reasons for believing that the nomic relation has the probability it does rather than some observed probability.

Thus, I propose further altering the nomic generalization. Where ' $\mathrm{p}$ ' is the probability that an $\mathrm{x}$ which is an $\mathrm{F}$ natured trope will have manifestation $\mathrm{M}$ after $\mathrm{x}$ is exposed to an s-stimulus, I write the following :

$$
\begin{aligned}
& \forall x\left\{(F x \& S x) \supset_{p} M x\right\} \\
& \forall x(\operatorname{Prob}\{M(x) /(F x \& S x)\}=p)
\end{aligned}
$$

The first line preserves our nomic relation, adding the probability $\mathrm{p}$. This probability $\mathrm{p}$ is defined by the second line. This second line says that for all $\mathrm{x}$ the probability of $\mathrm{x}$ manifesting $\mathrm{M}$, given that $\mathrm{x}$ possesses a maximally similar F-trope and $\mathrm{x}$ is exposed to stimulus $\mathrm{S}$, is equal to $\mathrm{p}$.

Finally, we can introduce the modal aspect of nomic generalizations for the dispositional essentialist. But this will require more than simply putting a " $\square$ " in front of the statement to symbolize necessity: we must be sure that we adequately capture the modal character of these laws. For the dispositional essentialist, the modality of laws of

\footnotetext{
166 If the given disposition of t-tropes when exposed to $S$ is accurate, we will of course expect these odds to trend towards $50 / 50$ as the sample size grows, but this is no guarantee that it will hit $50 \%$ and it may in fact be impossible for it to do so if, for instance, we use an odd number of particles.
} 
nature given probabilistic laws is of the following sort: given the sort of thing that $F$ is, when exposed to $\mathrm{S}$, the probability of $\mathrm{M}$ manifesting must be equal to $\mathrm{p}$. In the case where $\mathrm{p}=1$, that is, for deterministic laws, this translates to that given the sort of thing $\mathrm{F}$ is, when exposed to S, it must manifest M. But what does 'must' mean in these statements? It means that in being $\mathrm{F}$, F could not be exposed to $\mathrm{S}$ without having probability $\mathrm{p}$ of manifesting $\mathrm{M}$. Of course, this is still using modal talk. ("could," “must," etc.) But the goal here is not to provide an analysis of modality without using modal terms. Instead, it is to provide a way to understand the particular sort of modality that is present here and what grounds that modal nature. And it is the modal character of $\mathrm{F}$ that grounds the modality.

To symbolize this, I will use $\square_{F}$. This will change our nomic dispositional relation to the following notation:

$$
\forall \mathrm{X} \square \mathrm{F}\left\{(\mathrm{Fx} \& \mathrm{Sx}) \supset_{\mathrm{p}} \mathrm{Mx}\right\}
$$

This says for all $\mathrm{x}$ and for all worlds in which the modal character of trope $\mathrm{F}$ is respected, the probability of $\mathrm{x}$ manifesting $\mathrm{M}$, given $\mathrm{x}$ possesses a maximally similar F-trope and $\mathrm{x}$ is exposed to stimulus $\mathrm{S}$, is $\mathrm{p}$, i.e.:

$$
\forall \mathrm{x} \square_{\mathrm{F}}(\operatorname{Prob}\{\mathrm{M}(\mathrm{x}) /(\mathrm{Fx} \& \mathrm{Sx})\}=\mathrm{p})
$$

The chief difference here is to ensure that the content of dispositional essentialism is reflected in our formalism. By moving the symbolism from $\square$ to $\square_{\mathrm{F}}$ we are saying that the modal character of $\mathrm{F}$ is what grounds the necessity of the probabilistic conditional. To provide an example, the following notation of: $\forall x \square_{G}\left((F x \& S x) \supset_{p} M x\right)$ does not reflect any disposition, as this would claim that the modality of F is grounded in some other G. 


\subsubsection{Stimuli and Manifestations}

We must then ask what constitutes a stimulus and what constitutes a manifestation. Let's start with stimuli. At first glance, it is tempting to say something like "all stimuli are tropes or collections of tropes." While this will indeed be the case, we should examine some hard cases to see why. Take the following law: 'Caesium-137 has a half-life of 30 years." At the atomic level, this translates to, "For a collection of Caesium-137 atoms, on average each will release energy and decay into a lower-state particle in 30 years." Given this law, what is the stimulus here? It appears to be a spatiotemporal property and in particular the sort previously referred to as an area-property. This is notable because it is far from the typical sort of thing that we naively think of when discussing a stimulus. For in the case of radioactive decay it is not that there is some lower-level observable process that accounts for this average. It is instead a fact of Caesium-137 atoms. So it is not just true that tropes are stimuli, but that virtually any trope may be a stimulus for some other trope provided that the two interact in some way. A more problematic example of stimuli will be the case of where a law relies on a pseudo-trope such as an aggregate or an absence, particularly in the case of absences. As this occurs primarily in the case of ceteris paribus laws and ceteris paribus laws will provide us with a model of how to handle such cases, I will delay this issue until we can discuss ceteris paribus cases in more depth as they will be independently problematic. For now, it is enough to note that stimuli are tropes.

So what about manifestations? Using the idea that all manifestations are tropes creates its own problems. For while a manifestation may be a trope, there will be clear cases where we do not want to say that it is: particularly when the manifestation is the loss of a trope. Take the following sort of law, "Humans die from drinking arsenic." If there are 
tropes which constitute a human person, then at least some of those tropes are not present on the death of that person. As such, the drinking of arsenic has a manifestation which appears to not be the replacement of one trope with another, but which is rather the removal of a trope or set of tropes from existence. This creates a problem for manifestations as the manifestation is not itself a trope in this case but a lack of tropes.

To solve this problem, we need to examine how a trope, particularly an emergent trope, can be eliminated on this ontology. ${ }^{167}$ Remember that for emergent tropes there is some collection of lower-level objects which exist in particular relations that are sufficient for the existence of the trope in question. If the properties of a human person that are eliminated upon death are such tropes, then eliminating these tropes is a matter of eliminating either the constitutive parts or the relations between these parts in such a way that there is no structure that is sufficient for the existence of these tropes.

Consider the particular case of arsenic poisoning and the properties that make something a human person. While we may argue over some particulars, generally what makes something a human person rather than something else is thought to be things such as consciousness, autonomy, moral judgement, and the ability to meaningfully feel pain and pleasure are all thought to be important traits. Arsenic disrupts the production of ATP, resulting in damage to cardiovascular and neurological systems and, in higher doses, organ failure. Note that none of what arsenic does directly impacts any of the properties that we might think are important to one's being a human person. Instead, it disrupts the production of a chemical that is a part of the system that allows for those properties to

\footnotetext{
${ }^{167}$ I will remain quiet on the matter of whether or not foundational tropes can be eliminated for reasons mentioned earlier.
} 
exist. If one has taken a sufficient amount of dimercaprol, which acts to block the mechanisms that make arsenic toxic, arsenic has no effect on the tropes as it does nothing to disrupt the lower-level relations between parts that are sufficient for those tropes existence. So as long as we can refer to some set of lower-level manifestations that are not themselves absences, we may handle these cases while only using extant tropes. I know of no counterexample here, so this can handle the case of tropes leaving existence as a result of a disposition in response to a stimulus.

\subsubsection{Finks and Antidotes}

While antidotes were useful for demonstrating how one can explain manifesting something out of existence, antidotes have their own problems for the dispositional essentialist, as shown by Bird. ${ }^{168}$ The problem, roughly, is that antidotes only have a disposition when in a particular context. Take the following as the description of a genuine disposition: "dimercaprol prevents arsenic poisoning." Dimercaprol does not contain nor is it produced by arsenic, so there is no logical problem with imagining the existence of dimercaprol without the existence of arsenic. One can at least imagine that the toxic versions of chemical arsenic responsible for arsenic poisoning did not exist but dimercaprol does, or that due to spatiotemporal separation that there was dimercaprol but that none of it could effectively prevent arsenic poisoning. In what sense, in these cases, can it be said to have the disposition of preventing arsenic poisoning?

While metaphysical discussions generally do not need to go to the level of the physical sciences to undertake a description, in this case I think it can help provide us with

${ }^{168}$ Alexander Bird, “Dispositions and Antidotes,” The Philosophical Quarterly 48, No. 191, (Apr. 1998). 
useful principles. Take the case of a body that has been given a sufficient dose of dimercaprol prior to the introduction of arsenic. Once introduced arsenic normally chemically binds to thiols in the body resulting in the toxic effects described earlier. Dimercaprol prevents this as arsenic preferentially binds with the dimercaprol rather than the thiols and can then pass through the body harmlessly. So saying that dimercaprol has a disposition to prevent arsenic poisoning is a bit of a misstatement. A more accurate statement is that arsenic has a disposition to bind with dimercaprol preferentially over thiols and that once bonded, the arsenic-dimercaptrol does not meaningfully impact human anatomy. This implies that dimercaptrol is an antidote to arsenic, as when it is present in sufficient quantities the chemical reactions that make arsenic toxic to a human body do not occur. Of course, other sorts of antidotes exist. An antidote might affect the body to prevent the outside object from harmful interaction or it might disrupt the lower-level structures of the harmful object that make it so harmful.

But in each of these cases, it is not that the objects involved have some direct disposition to act as an antidote. Rather, it is that an object has a disposition to react with some other object, and that it just so happens that this disposition prevents another disposition from actualizing. To put it in terms of dispositional essentialism, the antidote has a disposition, when in the presence of a stimulus, to create a manifestation which in turn alters the environment such that the object to which it is an antidote is incapable of the effect it would have were the antidote not present. None of this requires that the substance to which it is an antidote actually be present or even exist: even if chemical arsenic did not exist or were not toxic to humans, dimercaprol would still react to certain structures in the way that it does and it would lose no disposition by virtue of a lack of 
arsenic: it would merely have an unmanifested disposition. And I know of no counterexample where an 'antidote' effect exists but where it does not either affect the environment to make the original stimulus not manifest or simply change the sufficient conditions that allow for one or more of the relevant properties to exist in the first place. So while some may think that antidotes are problematic for a dispositional essentialist, the dispositions of antidotes appear to be no different than other dispositions that are not considered problematic.

The problem of finkish dispositions, introduced by C.B. Martin ${ }^{169}$ can be solved by the combination of dispositional essentialism with constitutive trope theory in the same manner. A 'fink' is an object which gains a disposition whenever that disposition would be made manifest but does not have it otherwise. Martin uses the example of a machine which makes a wire live only when a conductor is touching it. Take it as given that a wire is live if and only if it is the case that if the wire is touched by a conductor electrical current flows from the wire to the conductor. And now we have a problem for the dispositional essentialist because the disposition of 'being live' can now be ascribed to the 'electro-fink' even when there is no current presently running through it. This is because if the conductor touched it then electrical current would flow from the wire to the conductor. As such, the electro-fink's wire 'is live' even though there is no current running through it on a standard conditional analysis of the causal power. But this seems absurd: the wire only becomes live when the conductor touches it, given the nature of the electro-fink.

As I stated, constitutive trope theory can handle this through examination of what it is that makes an object have the dispositional properties that it does. In the above sort of

${ }^{169}$ Martin, "Dispositions and Conditionals." 
case, what makes the electro-fink have the disposition to send electric charge to the conductor when activated has to do with the amount and structure of the electrons moving through it while it is active. That structure is not present when the conductor is not present. As the disposition is dependent on the underlying structure, there is no problem with saying that it is present when the conductor is present and absent when the conductor is absent. In fact, our intuition here is preserved: it is the current running through the wire that gives it the disposition at the time the wire is live. So a fink operates only through having a lower-level structure that gives it a disposition at some times and not at others. Here I not only know of no counterexample, a counterexample seems impossible. Finks exist by changing from lacking a disposition to having one at the moment of the stimulus that activates the disposition. Changes for emergent tropes are always a matter of changes to the underlying structures that are sufficient for the disposition, and so something's being a fink requires some change to those underlying structures. I suppose that it is possible that foundational tropes could be finkish. I cannot rule it out as I have no knowledge of what foundational tropes might look like. If this is a weakness of the theory, that it cannot explain foundational tropes that are finkish, then that is a bullet I have to bite.

\subsubsection{Ceteris Paribus Laws}

Ceteris Paribus laws create a different sort of problem for dispositions. As Bird helpfully points out, there are two kinds of ceteris paribus laws that are of concern to the dispositional essentialist ${ }^{170}$. The first sort is best described in terms of the phrase, 'all other things being equal.' For instance, water boils at $100^{\circ}$ Celsius, provided that the atmospheric

\footnotetext{
170 Bird, "The Dispositionalist Conception of Laws."
} 
pressure is $760 \mathrm{mmHg}$, or the average atmospheric pressure at sea level. So saying that water has a disposition to boil at $100^{\circ} \mathrm{C}$ is only true, 'all other things being equal.' The other sort is an exclusive sort of ceteris paribus law. For instance, mass causes objects to be drawn closer via gravitational forces. But this is only true in the absence of stronger forces, including stronger gravitational forces and also other forces such as electromagnetic and nuclear forces which may counteract the gravitational force of a given mass. So this second sort of ceteris paribus law is true 'in the absence of inhibiting factors.' The former is about other parameters being constant, while the latter is about there being no outside factor that contravenes the factors related in the law. For our theory, these are different insofar as one refers to other tropes whose interactions are necessary for the interaction but unmentioned by the law-statement, and the other refers to tropes which, if they were present, would alter the probability that the manifestation occurs. One place where this distinction becomes apparent is in the semantics of law-statements. A law-statement regarding the boiling of water should actually include both the temperature and pressure to be fully accurate, and the omission of pressure from its usual statement is only due to background beliefs about the pressure being sufficiently close to sea-level as to make no appreciable difference in our observation of the phenomenon. But in the example of gravitational force, the law describes what the situation would be like without any influence from other forces.

'All things being equal'-laws create one sort of problem for dispositional essentialism, while 'in the absence of inhibiting factors'-laws create another. The former is with the proper conditions being in place for the disposition to exist, while the latter is about conflict between dispositions. For the first, to have a disposition is to be disposed to 
behave in a certain way given certain conditions. If tropes are powerful properties in the sense that they are dispositional, then spelling out what those dispositions amount to is difficult if a trope only has a disposition given some set of 'all things being equal' (ATBE) conditions.

In order to solve the problem of ATBE-laws we need to separate out the ontological from the epistemic. In the case of ATBE-laws, there is a question about the stated law, such as "water boils at 100 degrees Celsius" and the actual properties of boiling, temperature, and pressure and their relations. The former is not of any real concern to metaphysics unless it is an accurate statement of the law. And while it may be true that in many cases water boils at 100 degrees Celsius, it is not accurate. In reality, while the property of water boiling is tied closely to temperature, it is also tied to pressure and other properties that are present for any given boiling event. To illustrate this, imagine an alien creature that lives on a world where the temperature is a constant 100 degrees Celsius, but where air pressure is not only much more varied than it is on earth but also that the alien creatures of this world are as adept at manipulating air pressure as we are at manipulating temperature. These creatures might state a law that "water boils at $760 \mathrm{mmHg}$ " and not mention temperature at all. Because the law-statement is about the features that are most often manipulated to create the property and not a statement of all of the properties that are a part of the sufficiency conditions for the supervening property to exist. That water's boiling is dependent on more than one property is no more a problem than its being dependent on a single property. That these properties must relate in certain ways is no particular challenge for this theory: it only means that accurate law-statements may require more complicated stories than merely functional law-statements. So the problem of 
ATBE-laws is merely a matter of law-statements, rather than of laws in the ontological sense.

'In the absence of inhibiting factors' (IAIF) laws create a different sort of problem. While the ceteris paribus clauses of ATBE-laws may simply be explained by law-statements not making mention of rarely manipulated properties, the ceteris paribus clauses of IAIF laws cannot be specified merely by adding conditions to a law-statement to make it more accurate. This is because in many if not most cases, the 'inhibiting factors' are not present in the standard property-interactions mentioned under the law. Take the law that 'gravitational forces pull objects together.' Now take the IAIF clause 'except when a stronger force overcomes this.' The former may describe a good many interactions without the clause being relevant. This is more problematic for a theory of tropes as the relevant interactions where the IAIF-clause is irrelevant, that is, where the disposition actually leads to its standard effect, by their nature do not have the factors relevant to the IAIF-clause. Given that properties are particularized, how can we account for tropes being dispositional if these dispositions are not efficacious?

To answer this, let's consider a standard law-statement form of 'all F's are G's' and add the IAIF-clause 'in the absence of H.' H might have one of two ways of producing this IAIF-clause. First, $\mathrm{H}$ might transform F so that $\mathrm{F}$ does not produce a $\mathrm{G}$. Consider the lawstatement, "When two animals of the same species copulate they will produce an offspring ${ }^{171 "}$ and add the IAIF-clause, "provided one of them is not sterile." In this case, the relevant H-property transforms the F such that it does not produce G. This case creates no

\footnotetext{
${ }^{171}$ Quite obviously, this would be problematic as an actual law, but for a given species it may be altered so as to be a law, if only a probabilistic one
} 
real problem for the dispositional essentialist who is also a trope theorist, I think. This is because in this case, the relevant F-property is not actually present. In our earlier example, the relevant property of fertility is not present in both animals. So on analysis, there is no problem. This would only be a problem if an H-property could transform F without changing its nature as an F. But to transform F is just to change its nature. So this creates no significant problem.

The other way that an H-property might alter the above is by interfering with the interactions that allow for the law. Take the case of gravitational attraction used earlier. Here it is not that the mass or some other relevant F-property of an object has been altered, but that there is some other force present that prevents the G-property from manifesting. To respond to this, I think we need to invoke a relation between $F$ and $H$ qua $G$ in a case where $\mathrm{H}$ prevents the manifestation of G. While we cannot invoke G itself without invoking negative properties, which we should want to avoid for reasons given earlier, we can invoke the interactions that allow $\mathrm{F}$ to give rise to $\mathrm{G}$. Consider the example of mass and attraction. Two distinct mass-properties may, through gravitational forces, cause their objects to approach one another if there are no stronger forces that keep them apart. What interaction accounts for this law? Given what we know from relativity theory, it is that these properties have the effect of distorting the space-time around their object, narrowing their trajectories. So let's consider the case where our IAIF-clause is invoked and the 'stronger force' is itself gravitational. Consider two masses, A and B, such that if there were no contravening forces their distortion of space-time would be sufficient for their attraction, but where there are two other masses, $C$ and $D$, such that $C$ attracts $A$ and D attracts B and where these pulls are enough to keep A and B apart. In this case, the 
interaction that is indicative of the law is not present: the space-time distortion around $\mathrm{A}$ that would provide its attraction to B is superseded by the space-time distortion that attracts it to $\mathrm{C}$, and similarly for B and D. Thus, once we analyze the mechanism for the interaction there is no problem. So in these sorts of cases, where for a $\forall \mathrm{X} \square \mathrm{F}\left((\mathrm{Fx} \& \mathrm{Sx}) \supset_{\mathrm{p}}\right.$ Gx), provided $\sim \mathrm{Hx}$ )' where $\mathrm{F}$ and $\mathrm{H}$ are similar properties, the problem dissolves under analysis of the interaction that gives rise to G.

Now consider the case where $\mathrm{F}$ and $\mathrm{H}$ are not similar properties. Take the case of an iron sphere atop an incline which is magnetized at the top, so that it fails to roll down the incline because of the magnetic nature of the incline it is placed upon. This sphere has two dispositions: one to roll down the incline owing to its mass, and another to stay in one place owing to its magnetic attraction. A scenario such as this, with two dispositions with contrary manifestations, can be formally represented as follows:

1) $\forall x \square_{F}\left((F x \& S x) \supset_{1} G x\right)$

For all $\mathrm{x}$ and all worlds containing $\mathrm{F}$, if $\mathrm{x}$ possesses an $\mathrm{F}$-trope and is exposed to stimulus $\mathrm{S}$, x manifests G with a probability of 1 .

2) $\forall x \square_{H}\left((H x \& S x) \supset_{1} I x\right)$

For all $\mathrm{x}$ and all worlds containing $\mathrm{H}$, if $\mathrm{x}$ possesses an $\mathrm{H}$-trope and is exposed to stimulus $\mathrm{S}, \mathrm{x}$ manifests I with a probability of 1 .

3) $\forall x \square_{G} \square_{I} \sim(G x \& I x)$

In all worlds containing G and I, G-tropes and I-tropes do not manifest together.

4) $\forall x \square_{F} \square_{H}\left(\{(F x \& H x) \& S x\} \supset_{1} I x\right)$

For all $\mathrm{x}$ and all worlds containing $\mathrm{F}$ and $\mathrm{H}$, if $\mathrm{x}$ possesses $\mathrm{F}$ and $\mathrm{H}$ and is exposed to $\mathrm{S}$, $\mathrm{x}$ manifests I with a probability of 1 . 
5) $\exists x(F x \& H x)$

There exists some object that has both F and $\mathrm{H}$ tropes.

6) $\diamond s \exists x\{(F x \& H x) \& S x\}$

Finally, given the nature of stimulus $\mathrm{S}$, it is possible for an object to have $\mathrm{F}$ and $\mathrm{H}$ and be exposed to $\mathrm{S}$.

From here, we get a contradiction, provided that an object which possesses $\mathrm{F}$ and $\mathrm{H}$ is exposed to $\mathrm{S}$. This is because there is an object with tropes $\mathrm{F}$ and $\mathrm{H}$, and should this object be exposed to $S$ it will, by 2 (as well as 4) manifest I. But by 1 , it will manifest G. But G and I cannot manifest together. We might attempt to avoid the contradiction by stipulating that no such cases may occur, but as this is based in actual cases this does not seem a feasible strategy. Instead, the key lies in point 4, above, which shows that the disposition of $\mathrm{H}$ dominates the disposition of $\mathrm{F}$ with respect to $\mathrm{S}$. "With respect to $\mathrm{S}$ " is important here. Consider the example with the iron sphere. If the top of the incline were magnetic but lacked sufficient pull, it may not serve to restrict the sphere from rolling down the incline. Yet without the incline, the magnetic force might be sufficient to keep the sphere in place. We can express this if we include the following our formal understanding of the modal nature of the disposition:

$\forall \mathrm{x}\left(\left(\left(\mathrm{Sx}_{\Phi} \& \mathrm{Sx} \Psi\right) \&\left(\Phi_{\square}>\Psi_{\square}\right)\right) \& \forall \mathrm{y}\left(\sim \diamond\left(\mathrm{My}_{\Phi} \& \mathrm{My}\right)\right) \supset \mathrm{My}_{\Phi}\right)$

This says that given two stimuli, $\mathrm{x}$-phi and $\mathrm{x}$-psi, where phi necessarily overpowers psi, and for a manifestation y such that it cannot manifest both manifestation y-phi and 
manifestation y-psi, only the manifestation of y-phi will manifest. 172 Of course, which disposition dominates for a given stimulus or manifestation will depend on the particular dispositions and stimuli in question, as we have seen. But our goal was not to solve the problem of which dispositions dominate when, but rather to give a formal explication of the modality of one disposition dominating another.

\subsubsection{Weak Realism About Laws}

In Chapter 2's discussion of laws, I distinguished between three views on the ontological status of laws. To reiterate these positions:

Strong Realism of Laws: Laws of Nature are unique ontological entities in their own right.

Weak Realism of Laws: Laws of Nature are grounded in actual ontological entities but are dependent for their nature on them.

Antirealism of Laws: Laws of Nature are not ontologically grounded, but may be grounded in some other way (epistemology, methodology, sociology, etc.)

As discussed earlier, both Strong and Weak Realism will grant us ontologically significant laws of nature. We can see now that the theory presented here is a Weak Realism rather than a Strong Realism. Laws on my account are not an entity: they are not even a kind of trope. However, given the similarity between tropes, the granting of tropes to be powerful

\footnotetext{
172 We could include versions of this for cases of increasing numbers of dispositions, but this is not necessary as one disposition being dominant over another is transitive: if disposition a dominates disposition $b$ with respect to $\mathrm{s}$, and disposition $\mathrm{b}$ dominates disposition $\mathrm{c}$ with respect to $\mathrm{s}$, then disposition a dominates disposition $\mathrm{c}$ with respect to $\mathrm{s}$.
} 
properties, and the actual interactions of tropes with each other, we can derive laws of nature from the behavior of tropes. As this derivation is not grounded in an epistemic or methodological consideration but is instead grounded in the actual similarity and dispositional nature of tropes, this amounts to a Weak Realism of Laws.

One may object that only Strong Realism about laws grants us ontologically significant laws of nature. Mumford makes this argument in his eliminative account of laws of nature:

What is essential for a theory to be nomological realist is that laws are understood as an addition of being. They must be something more than the regularities or patterns that are to be found in the world. If these anti-Humeans are right, and the necessary connections in nature are laws, then such an account has good credentials for being a theory of laws: a theory of nomological realism. Laws are seen as an addition of being that, it might be maintained, play some role in determining their instances rather than being entirely constituted and exhausted by their instances. ... Furthermore... (these theories) must be arguments for something more than regularities or patterns. Nomological realism is a metaphysically stronger claim than Humeanism. It claims that there is something in the world that Humeanism denies. ${ }^{173}$

In response I will address what I take to be two different points of the argument. The first is that laws of nature are not necessary. The second is that their inclusion is primarily historical. I take this second point to be the weaker of the two. Even accepting that laws of nature have been utilized primarily for the sake of explaining the success of science for categorical properties and not for modal properties, ${ }^{174}$ that in and of itself does not indicate that they have no place in a dispositional essentialist account of properties. At best, this

\footnotetext{
173 Stephen Mumford, Laws in Nature, (Routledge, Routledge Studies in Contemporary Philosophy, 2002): 678.

${ }^{174}$ Mumford talks of 'powerful properties' as being modal, rather than 'categorical' as their dispositional nature allows them to ground modal truths.
} 
may cause us to question if such an account of properties needs laws, given that in other accounts laws fill a gap that is not needed for the dispositional essentialist.

For the first point, we must distinguish between laws of nature being logically necessary, ontologically necessary, or epistemologically necessary. Given other arguments he makes in favor of examining laws of nature in terms of ontological necessity, I take this to be Mumford's primary line of attack. The claim then is that to justify laws of nature in our ontology those laws must be de re necessary for some facet of the world. For instance, one may think that certain regularities could not be the case without some law of nature to make the regularity occur. As Mumford accepts 'powerful properties' 175 but rejects laws we may take his account to be particularly damning. If powerful properties sans laws can perform the ontological work of grounding scientific practice without talk of laws, then why include laws, particularly if the laws are not themselves ontologically basic but only exist as a consequence of the dispositional nature of other properties?

Here we need a further distinction between two different notions of a law of nature: ontological and methodological. I take it that Mumford is right about ontological laws of nature, that is, laws of nature as entities. Postulating an entity that is a law of nature if one is unneeded for a particular account should be avoided on grounds of simplicity. However, this is not the notion of law of nature that has been defended here. What has been argued for in our desiderata is only that an account of properties should be able to account for laws of nature and ground them in some ontologically significant entities. It has not been argued and is in fact an opposing position to this account that laws of nature themselves

\footnotetext{
${ }^{175}$ Although he is quiet on whether these properties are tropes or universals and at some points seems to embrace the idea that they are or could be universals, making his account notably distinct from this one.
} 
need be ontologically significant entities. Instead, much as we can talk about an 'object' wherever we have a colocation of properties, we can talk about a 'law of nature' whenever we are describing the dispositional nature of some property. As laws of nature are not independent entities on this account but are instead grounded in the existence of ontologically extant entities, namely tropes, there is a place for their inclusion in one's ontology even if they are not themselves extant. It is the methodological laws of nature that need be justified on this account and while one way of doing so would be to show that those laws of nature are themselves ontological entities, it is as-good a solution that accurate law-statements are grounded ontologically.

\subsubsection{Problems for Dispositional Tropes}

If a trope theorist takes the above position there are a few bullets that he will need to bite. As mentioned earlier, the trope theorist will have to dismiss Humean intuitions. This means that the trope theorist who wants to utilize dispositional essentialism will have to admit that rather than tropes being merely categorical that for each trope there is something inherent in the nature of the property itself that gives it lawful relations to other tropes. To use the language of Bird, tropes on this view will have to be 'powerful properties.' Notably, on this theory tropes are categorical but ground modal dispositions. In this way, they are 'powerful properties' though not in the way that a dispositional monist such as Bird would have it. Second, a general worry for dispositional essentialism has to do with properties which are intimately tied but have different 'essential natures.' Take the example of inertial mass and gravitational mass. An object's inertial mass and its gravitational mass are always equal and one might be tempted to say they are identical. 
However, this creates a problem for the dispositional essentialist as there appears to be no logical reason that this is so, nor any bridge law that explains this equivalence. ${ }^{176}$ While this is a general problem for the dispositional essentialist, I believe this version of the 'mixed view' resolves it as tropes do not reduce to dispositions but instead have a dispositional aspect. So a single trope of 'mass' can lead to both certain inertial dispositions and certain gravitational dispositions. As there are not two properties but one, the problem dissolves.

A further problem specific to this theory can be raised, given that this is a mixed categorical-dispositional theory of properties. That is to say, that tropes have both a modal and non-modal nature. While a precise version of this has been given, one might still wonder what it is about the categorical tropes that grants them a modal character. Similar to Lewis' objection to Armstrong that merely calling something 'nomic necessitation' does not impart any amount of necessity, one could worry that merely saying that tropes are dispositional does not resolve the problem of how they can ground modal facts without giving way entirely to dispositional monism. That is to say, if tropes ground modal facts but are not merely dispositions, how do they do so?

To begin to address this problem, let's consider, in addition to mixed tropes, the possibility of purely categorical or purely dispositional tropes. The problems of the latter have already been discussed, and on the current theory seem ruled-out as dispositions are not taken to be some entity but are instead a facet of a trope. So let's consider the possibility of a purely categorical trope. By a purely categorical trope I mean a trope with no dispositional character. Interestingly, there is no way to show that such tropes exist. For

\footnotetext{
176 While the equivalence principle of general relativity assumes that they are the same, this is an assumption of general relativity rather than a proof that comes out of it.
} 
any evidence of the existence of a trope occurs in some manifestation. ${ }^{177}$ A purely

categorical trope, one with no dispositions whatsoever, would at minimum be empirically undetectable. Further, such tropes could not act as a part of the constitutive basis for any further tropes, as there would be no stimulation:manifestation-relation that would allow ${ }^{178}$ them to be relevant to the constitutive parts of a trope. There is no reason they could not exist as emergent tropes: we could imagine a set of mixed tropes giving rise to a categorical trope, but such a trope would be an ontological dead-end. It would result in nothing, be impossible to notice, and have no interaction with the world beyond those that result in its constitution. A purely categorical foundational trope would lack even this. As such, while there is no real harm in admitting such tropes, there is also no reason to accept them into one's ontology. Given Occamist concerns, we may then harmlessly eliminate purely categorical tropes from our theory. Combined with the problems that arise from purely dispositional tropes, this means we may restrict ourselves to talking about mixed tropes.

But even in the case of dispositional tropes, we can imagine that they manifest no dispositions. Imagine a world where there exist a number of fundamental, mixed tropes: [A, B, C... N], such that A-N lists all tropes present in the world. ${ }^{179}$ For each trope in this world,

\footnotetext{
177 One may object that this only works for physical tropes, but not for properties attached to entities such as numbers, propositions, etc. I am generally suspicious of bringing such entities into my metaphysical landscape, but if we take them to be genuine properties than they will still carry dispositions. As an example, take the numerical property of being odd. Odd numbers, when added to non-zero even numbers, produce a new odd number, and when added to an odd number produce an even number. Thus, odd numbers are disposed to behave in certain ways in addition. Similar examples can be crafted for propositions and other abstracta. One may question whether or not these count as genuine dispositions: I am sympathetic to this worry, but believe it is the same sort of worry that leads me to be suspicious of numbers, propositions, and other abstracta as entities at all.

178 They could be a part of an object that is a constitutive part, but they could not feature prominently in any of the relations that give rise to an emergent trope, as this would amount to having a disposition. 179 [A... N] must be foundational, as if any were emergent there would be some manifested disposition: namely, the disposition to manifest a particular trope when in the presence of other tropes.
} 
call its disposition $d$ such that the exhaustive list of dispositions is [dA, $d \mathrm{~B} \ldots d \mathrm{~N}]$. For each $d$, take its stimuli to be drawn exclusively from a list of tropes of the set [a, b, c... k], all of which at this world are possible but not actual. Thus, no $d$ ever leads to a manifestation, as the only stimuli that 'activate' the dispositional nature of the tropes in this world are themselves non-actual. This not only means this world lacks emergent tropes, but it lacks any lawful interactions between tropes whatsoever.

Such a world may be possible, in the sense that there is no contradiction or conflict with natural laws in assuming that such a world exists. But it cannot be actual, or at least, it cannot be actual for any world containing human beings, as human beings require emergent tropes for the biology, chemistry, and physics that make up our being. This gives us anthropic reasons to find ourselves in a world of mixed tropes with active dispositions. For even if a mind could exist as a fundamental entity rather than an emergent entity, such an entity would exercise no causal powers or interactivity with anything else in its world. ${ }^{180}$ So while tropes with no dispositions or whose dispositions never manifest may be possible, in our own world they are ontological dead-ends at best, and a world composed of nothing but these entities could not resemble our actual world. Thus, for our actual world the simplest answer is to treat all tropes as mixed tropes, as any tropes without dispositions which may manifest given the other properties of the world matter nothing to anything else in the world they occupy.

\footnotetext{
180 It may seem odd to think of a mind as lacking any disposition, or for tropes to compose a mind without any of those tropes being dispositional. If you believe this to be problematic or impossible, I am happy to accept that and only include it here to address a potential objection to an anthropic reason to favor a mixed-trope world.
} 
This reduces the requirement for the mixed-view. As both in-world purely categorical tropes and worlds composed of nothing but purely categorical tropes may be discounted as possible but unable to produce something like a human person, it only needs to be possible that tropes have a dispositional character that is grounded in the sort of thing that they are. And there seems to be no good reason to deny this: in fact, this follows the pattern of how we find things in the world. Properties exist and are disposed to act in certain ways, and there is no contradiction in them doing so. Their possibility is evidenced by the fact that we do observe objects to have properties, and that the having of a property leads one in some cases to accurately predict the dispositions that the object possesses. As mixed-tropes are possible, purely categorical tropes are uninteresting and purely dispositional tropes are problematic, we may assume the world to consist in tropes with a mixed dispositional and categorical nature with their modal aspect grounded in a nonmodal nature that each trope possesses. 


\section{Chapter 5: Problems and Final Considerations}

Now that we have a theory of properties and how to ground the truth of scientific law-statements, we may examine in greater detail some of the unanswered problems for the theory. As a reminder, our core theory is an ontology in which tropes feature as the only irreducible and ineliminable elements of the theory. Each trope is either foundational or emergent. Little has been said about foundational tropes, but more can be said about emergent tropes, which represent most if not all of the tropes we are actually familiar with. Emergent tropes are dependent on relations between parts which are sufficient for their existence. As these structures are often responsible for more than one property, this explains why it is that emergent tropes 'bundle' together into objects. In the actual world many parts arrange in qualitatively identical configurations. Further, there are occasions where more than one structure may be sufficient for the presence of a given trope. While it is possible to get a world like this without constitutive trope theory, this does explain why we get many exactly resembling tropes. The parts sufficient for tropes are themselves objects composed of trope bundles. If the tropes which compose the bundle are also emergent there will be yet another layer upon which the parts are dependent. Further, tropes are not mere categories on this theory: not merely a charge or mass or something else without any modal character. Tropes are inherently dispositional: a disposition is a part of the nature of a trope, rather than an independent entity of some kind. Accurate scientific law-statements describe the dispositions of tropes. Hence, the truth of scientific laws is grounded in the dispositional nature of tropes. 
There are at least three matters that still need to be addressed. First, there are problems which must be answered if this theory is not to fail painfully with respect to our fifth desideratum. Second, this theory needs to be placed within context of the greater trope literature so that it may be accurately assessed. Third, I would like to show one advantage constitutive trope theory may have over its competitors in that it is in principle empirically falsifiable.

\subsection{Problems for the Account}

It is impossible to be sure that every single problem for a theory has been examined. Even if one believes they have examined a full accounting of the problems for a theory, some new problem might be discovered by others in the future. However, we can look at the problems and failings of other theories and look for potential similar weaknesses in a theory that we examine. There are four objections that I believe constitute the major objections to this theory. First is its treatment, or lack thereof, of foundational tropes. Second is that the possibility of single-trope objects. Third is a complaint the realist can raise against the dispositional account of laws of nature. Fourth, there is an objection that can be raised regarding this theory's explanation for similarity. Lastly, Michael Loux brings several objections against the bundle trope theorist which may be relevant here. While I do not think that any of these are knock-down objections against the trope theory presented here, I do think that each needs to be addressed for the theory not to be overwhelmed by the fifth desideratum. 


\subsubsection{Foundational Tropes}

The most difficult objection, I believe, regards this theory's lack of treatment of foundational tropes. The objection may go something like this: even if the theory does all of the work we desire a trope theory to do for emergent tropes, it does not handle foundational tropes at all. As emergent tropes are dependent on foundational tropes for their very being, not providing an account of foundational tropes makes the whole account fall apart. Without this foundation, the whole edifice is unstable, and it is sorely lacking. Moreover, the explanation of compresence that works for emergent tropes does not work for foundational tropes. Compresent emergent tropes are explained by their dependence on lower-level objects, but compresent foundational tropes cannot be explained in this way. So there are at least two major problems for constitutive trope theory on this objection. To combat these objections, I will bring in three lines of defense. First is that there might not be any actual foundational tropes. Second is that if there are, we ought to be hesitant about attaching beliefs about them. Lastly, that the sufficiency criterion for trope instantiation takes care of most if not all of the problems with this version of the theory.

The first point is one of the possibility of the world being composed of 'atomless gunk.' This view is defended in places by Tahko, ${ }^{181}$ Zimmerman,, ${ }^{182}$ and others. The basic conclusion of these arguments is that the objects of the world may always be sub-divided into smaller proper parts and that reality does not 'bottom out' on any level. In a gunky world there would be no foundational tropes, as each trope would have a lower level on which it supervenes. Thus, in a gunky world, the problems involving foundational tropes

\footnotetext{
181 Tuomas Tahko, “Boring Infinite Descent," Metaphilosophy, 45, No. 2, (Apr. 2014).

182 Dean W. Zimmerman, "Could Extended Objects Be Made Out of Simple Parts? An Argument for "Atomless Gunk"," Philosophy and Phenomenological Research, 56, No. 1 (Mar. 1996).
} 
can be discounted as there are no such entities. This does not mean that a defender of constitutive trope theory needs to believe in a gunky world: all that is necessary is that the gunky world is possible and that constitutive trope theory does not itself rule it out. If gunky worlds are possible, then constitutive trope theory need not be prejudiced against them. If gunky worlds are impossible for reasons unrelated to constitutive trope theory, then they are still possible qua constitutive trope theory and so that is still not a reason for constitutive trope theory to be prejudiced against them. Opponents of gunk might think that constitutive trope theory would be better if it could rule out the possibility that we live in a gunky world, but provided this possibility is alive there is no reason for the constitutive trope theorist to be committed to foundational tropes existing at all, much less provide an explanation of them.

But there is a problem for the constitutive trope theorist who wishes to accept gunky worlds. Gunky worlds are typically defended by taking objects to be mereologically divisible regions of spacetime, as Zimmerman does. ${ }^{183}$ But the objects of our theory are not of this kind: they are defined in terms of compresent properties, not as regions of spacetime. If the justification for gunky worlds relies on objects as defined by this mereology, then that defense will fall flat for the constitutive trope theorist. This I will grant. But theories of a gunky world do more than just assert the possibility of gunky worlds: they attack the notion of ontological well-foundedness, as Schaffer puts it. ${ }^{184}$ Ontological wellfoundedness is the notion that chains of dependence must terminate in a fundamental supervenience base. And as defenders of gunky worlds have noted, ontological well-

\footnotetext{
183 Ibid.

${ }^{184}$ Jonathan Schaffer, “Monism: The Priority of the Whole," Philosophical Review 119, No. 1, (2010).
} 
foundedness is hard to defend as more than a mere intuition. ${ }^{185}$ Without such a defense, a world entirely composed of emergent tropes with no bottom level is still a possibility even if objects are not defined terms of spacetime regions for constitutive trope theory.

Even though this line of defense for constitutive trope theory is promising, I do not think it is likely that we live in a gunky world. Thus I prefer defending that foundational tropes are not objects of which we can speak, as we lack knowledge of them. This comes down to a matter of justification. If we have justified beliefs about foundational tropes, these believes will be justified either on a priori grounds, a posteriori grounds, or some combination of the two. These beliefs cannot currently be justified a posteriori, as a posteriori justification is based on our experiences and we have no experience with any properties that might be foundational. In past eras when scientists took various objects and their properties to be foundational (the atomic level, the sub-atomic level, etc.) each time scientists have discovered yet another layer below the one they took to be foundational. This provides some reason to be skeptical that the quantum level is the lowest one, even if we have yet to discover what underlies it. Scientists seem to be taking this approach as well. Even now, there is significant research into what lies underneath quantum phenomena. Physicists are actively considering the possibility that spatial and temporal properties may be grounded in some yet more primitive properties. And there is no guarantee that the level below the quantum will be the foundational level either. This being the case, we have no experience with foundational tropes and so cannot be justify our beliefs about them a posteriori.

185 Tahko (2014) presents such an argument, as does Zimmerman (1996) 
This leaves a priori justification, but I do not think this is promising either. What sorts of properties exist is not the sort of thing one can merely determine a priori. While I believe a priori reasoning has its place in metaphysics and fundamental physics both, the nature of extant properties is not something that can be determined solely through a priori methods. It is not clear how one could discover properties such as tensile strength or light reflection through purely a priori processes, or even more fundamental properties such as charge. And there are reasons to think that the sorts of properties that underlie the quantum level will be novel, as the quantum level itself is novel. Prior to the scientific discoveries of quantum mechanics, philosophers did not a priori discover that quantum laws were probabilistic. Yet today, any philosopher attempting to ground nomological truths cannot ignore probabilistic laws in their account. If these sorts of novel properties and novel relationships between properties are possible, then that gives us even more reason to adopt a position of epistemic humility regarding foundational tropes, which may be entirely unlike the properties we are used to.

But a philosopher accepting epistemic humility here invites a criticism that we might need to adopt epistemic humility with the whole project of finding properties. If undiscovered properties are suspected to be novel and unlike known properties, then we may not be justified in reasoning about properties at all. It is not even a matter of foundational tropes at this point: there may be layers between the known and the foundational which operate in a manner such that we may rule out this entire project. There are two responses to this worry. The first is that it is not a worry unique to constitutive trope theory: future discoveries threaten any enterprise which may depend on their results, and this is unique neither to a theory of constitutive tropes or even ontological 
theories in general. But even if constitutive trope theory fails at some future date, I believe the theory can account for all currently known properties and this is all one can ask of a theory.

\section{$\underline{\text { 5.1.2 Single-Trope Objects }}$}

A puzzle arises for bundle theories with respect to single-trope objects. If an object can consist of a single trope, this calls into question what the distinction is between a single-trope object and the trope that composes it. The trope theorist can either admit or deny the existence of single-trope objects. If an object cannot exist as a single trope, the trope theorist must defend against this possibility. If single-object tropes can exist, what makes them such must be defended. While, as we will see later, constitutive trope theory is not quite a bundle theory, it may be vulnerable to this puzzle in two different ways: singletrope objects consisting of a foundational trope, and single-trope objects consisting of an emergent trope. I will address the puzzle of emergent tropes first as I take it to be the more pressing objection.

On constitutive trope theory, many properties of non-foundational objects are not strictly speaking tropes but are instead derived from the properties of their parts. For instance, the masses of objects other than elementary particles are aggregates of the masses of their parts and the location of an object is derived from the location of its parts. This opens the door for objects consisting of a single emergent trope. In fact, such objects seem likely on constitutive trope theory: all that is required is for the constitutive parts in question to be sufficient for the instantiation of one property but no others. And there seems to be nothing in the theory to prevent the existence of such objects. But if 
constitutive trope theory allows for such objects, what separates the abstract particular, the trope, from the concrete particular, the object?

To answer this, let's assume the existence of a single-trope object. Call this object $Q$, its only trope q, and the parts and relations sufficient for q P. As a concrete particular, $Q$ cannot share its substrata with any other object. Further, as an object is merely a compresence of tropes, $Q$ is the compresence of the set of its tropes, whose sole member is q. ${ }^{186}$ But let's imagine that $\mathrm{P}$ were arranged slightly differently, call this $\mathrm{P}^{*}$. Like $\mathrm{P}, \mathrm{P} *$ is sufficient for the existence of q. But it is also sufficient for the existence of another trope, $r$. If $\mathrm{P}$ is rearranged as $\mathrm{P}^{*}$, this change results in $\mathrm{Q}$ adopting the trope $\mathrm{r}$. This change does not destroy $Q$, as it is still a single compresence of tropes. This shows that $Q$ cannot share all and only P with another object, but q can. This distinguishes between $\mathrm{q}$ and $\mathrm{Q}$ while allowing for single-trope objects. As the compresence relation does not require a bundle of multiple tropes on constitutive trope theory, there is no problem with having single-trope objects exist in this way.

But can a single-trope object consist of a foundational trope? This is actually less problematic for constitutive trope theory than multi-trope objects consisting of foundational tropes. Constitutive trope theory has no mechanism for explaining the case of foundational trope compresence: like other trope theories, such a compresence would have to be taken as a primitive and would call into question whether or not constitutive trope theory is superior to its alternatives. However, if objects composed of foundational tropes

\footnotetext{
${ }^{186}$ One may argue that $\mathrm{q}$ cannot be in a relation of compresence if there are no other tropes for it to be compresent with.There are problems for this view, as it requires that objects have at least 2 genuine tropes per object and may allow for "free floating" tropes: tropes without objects. However, if one does not think that a single trope by itself can be an object, then the problem of single-trope objects for constitutive trope theory dissolves.
} 
are each composed of a single foundational trope, this problem dissolves. But as our previous allowance for single-trope objects depended on differences in substrata, the problem arises anew for single-foundational trope objects. Further, it may be a necessary truth about foundational tropes. As constitutive trope theory has not done much to characterize foundational tropes, it is possible (perhaps even likely) that if they compose single-trope objects they do so necessarily. This would force constitutive trope theory to accept primitive compresence relations, if it holds.

This might not be an indictment of constitutive trope theory. For instance, it may turn out that either there are no foundational tropes or that the objects of foundational tropes are multi-trope objects, saving constitutive trope theory from this problem. But as stated, single-foundational trope objects otherwise solve problems for the theory and so an answer here would be helpful. This problem can be solved provided that single-trope objects of foundational tropes are not metaphysically necessary. That is, if there is no contradiction in positing a world where multi-trope objects form at the foundational level, where there are no foundational tropes, or some other option for foundational tropes is present. It might still be physically necessary: a world like ours, with the physics that our world has, might be required to have single-foundational trope objects at its most basic level. But as long as the fact that this sort of object is foundational is a contingent fact, this problem for the constitutive trope theorist is held at bay, as the distinction between trope and object can still be maintained. So single-property objects, while problematic for bundle theories generally, are not problematic for constitutive trope theories qua emergent tropes and the problem qua foundational tropes can be mitigated in a number of ways. 


\section{$\underline{\text { 5.1.3 Dispositions vs. Universals }}$}

There is another objection the realist can raise against constitutive trope theory given the theory's reliance on dispositions to handle our third desideratum. Universals were rejected as unnecessary: the relevant issues in philosophy and science regarding properties can be handled quite neatly without positing them. However, tropes ended up being dispositional on this account. Yet this creates a problem of theory choice: why accept dispositions and reject universals? Both appear to add to the complexity of the account, and there may be reasons in favor of universals. Even if universals are not more desirable than tropes and dispositions, at minimum it appears that the trope theory loses its advantage of being a single-category ontology.

The clearest response to this is in that unlike the addition of universals, dispositions are not taken as an entity independent of tropes. Rather, it is taken as a part of a trope's nature that it is dispositional: that tropes are such that they are predisposed to causal changes if certain environmental conditions are met. As dispositions are not an independent entity on this thesis, but merely a characteristic of tropes, the objection is diffused.

But the objection may prove problematic if dispositions can be located in nontropes. While constitutive trope theory only admits tropes as fundamental, irreducible entities of its ontology, there are entities that exist as constructions of tropes (objects) as well as pseudo-tropes. (such as aggregates) While non-entities do not 'exist' ontologically, there are some (such as absences) which can be descriptive of parts of reality. If these entities can also bear dispositions, then the problem can be pressed as dispositions can exist apart from tropes, indicating that they are a different sort of entity. For instance, if 
absence can be properly said to bear a disposition, or if an object can bear a disposition that its tropes do not bear, then constitutive trope theory will have a significant problem.

Objects as the bearers of dispositions may prove a particularly tricky case as typically we speak of objects, not properties, having dispositions. For instance, we normally would say a wheel may have a disposition to roll if left on a slope, not that its roundness does. That it is intuitively plausible therefore that objects and not their properties are the proper bearers of dispositions is something that constitutive trope theory must answer. This case may be made more plausible as the having of a disposition might require several properties. A wheel left on a slope won't roll without the mass of the wheel, another mass in an appropriate relation to the slope, the roundness of the wheel, etc. But if the object can bear a disposition that its tropes do not, this indicates there is something more to the object than a mere bundle of tropes.

A constitutive trope theorist can respond by pointing to what grounds the disposition. The disposition of a wheel to roll when left on a slope is not merely taken to be a fact about the object simpliciter. It is because it has various properties: mass, roundness, etc.; and because it is in a particular set of circumstances that it has this disposition. So while the object may be what actually acts, any dispositions are entirely grounded in its properties. We can further press this by showing how a single object may have competing dispositions. Assume for a moment that our wheel is made of iron and at the top of the slope there is a powerful magnet holding it in place. The magnetic susceptibility of the wheel makes it disposed to be attracted to the magnet at the top of the hill, while its mass and shape give it the disposition to roll down the hill. Depending on the particular strengths 
of the forces involved, the wheel may or may not roll down the hill. ${ }^{187}$ If the dispositions are grounded in the object, it is less clear how an object can have competing dispositions in this way. But dispositional tropes can explain competing dispositions in a way that dispositions simpliciter grounded in objects cannot.

But what of pseudo-tropes and nonentities? These are not tropes, so if they bear genuine dispositions a problem arises. A color aggregate is an example of an entity that is a pseudo-trope that may pose a problem here. The colors of macro-level objects, such as a table, are not genuine tropes. Rather, they are aggregates of lower-level collections of tropes at the level of the color absorption of the constitutive molecules of the object. If a herpetologist describes the coloration of a snake as giving it a disposition to be hidden in its native environment, then straightforwardly this would involve a pseudo-property being the bearer of a disposition. Clearly, this is an untoward consequence. But the constitutive trope theorist can block this by use of the individual, smaller color tropes. Each color trope has its own effect on the eyesight of predators, and the combination of color tropes in the patterns they are in is what masks the snake's presence in its native biome. So it is each of the color tropes, in conjunction with the system of spatial relations that they find themselves in and combined with the shape of the snake that bears the disposition. As the relation between these properties is itself a trope, that relation is capable of conferring the disposition towards stealth to our snake.. As pseudo-tropes are themselves grounded in tropes, the relations between the tropes that ground them can ground laws which reference the pseudo-trope.

${ }^{187}$ For a larger discussion of conflict between dispositions of varying strengths, see Chapter 4. 
Lastly, nonentities such as absences are perhaps the easiest of the kinds to deal with. While the pseudo-property of being an absence has already been discussed, we have not considered the case of a total absence: an object with no properties. Consider the case of an empty region of space, which is perhaps the prototypical example we would point to of an absence. ${ }^{188}$ There are only two options: either this space has tropes or it does not. Having a disposition implies having some sort of trope: a genuine property. If the absence does not have any tropes, therefore, there is no problem of absences bearing dispositions. If an empty region does bear tropes, however, then it is not a true absence. Its only properties may be geometric or similar in character, but if these are tropes it will not be an absence according to constitutive trope theory. If empty regions of space are not absences, it is not clear what could count: if a region that is defined by a lack of anything has tropes and every region with things in it is ultimately composed of tropes, then there is no place in the universe that is empty of tropes. As such, either absences do not exist to be the bearers of dispositions or they exist but cannot have dispositions.

\subsubsection{Loux's Objections}

Michael Loux presents four problems for bundle trope theories. Constitutive trope theory is not strictly speaking a bundle theory but may be vulnerable to the same criticisms. Recall that while objects on constitutive trope theory are bundles of tropes, the constitutive parts of non-foundational operate as a substratum. This renders constitutive

\footnotetext{
${ }^{188}$ According to physics, the implications of virtual particles and quantum fields may lead us to doubt the existence of true 'empty space.' If there are no such spaces, then absences may not exist and the problem dissolves. Therefore, we will assume that such spaces exist for the sake of providing the best argument against constitutive trope theory.
} 
trope theory close enough to a typical bundle theory that it requires addressing these problems, as I will attempt to do here.

Michael Loux presents these problems in the following manner:

(1) That the theory entails the truth of the Identity of Indiscernibles;

(2) That the theory commits us to excessive essentialism (what I have called ultraessentialism);

(3) That the theory cannot accommodate our pre-philosophical idea that things persist through change;

(4) That the theory cannot explain the individuality or concreteness of familiar particulars. ${ }^{189}$

(3) has already been addressed, and treated with seriousness, as the idea that things persist through change is present in scientific law-statements and so is treated as more than merely a pre-philosophical idea. To recount it briefly: as emergent tropes are grounded in the relations between their parts and these parts may persist even if one of the tropes they previously instantiated fails to due to a change in their relations, an object may persist despite change provided that the constituent parts which instantiate its tropes retain some relations that result in some emergent tropes. This provides us with an answer to Loux's (2) as well. Loux rephrases this second problem clearly when he says of bundle theory, “... it commits us to an excessive form of essentialism where every property associated with a familiar object turns out to be essential to it." 190 As the object exists so long as parts sufficient for some of its constitutive tropes exist, it is not the case for constitutive trope theory that every property associated with a familiar object turns out to be essential. For objects existentially reliant on constitutive parts, what is essential to an object's continued existence is only that some number of emergent tropes of its parts obtain.

189 Michael Loux, "An Exercise in Constituent Ontology" In The Problem of Universals in Contemporary Philosophy, ed. by Gabrielle Galluzzo and Michael Loux, (Cambridge, Cambridge University Press, 2015): p. 945. 190 Ibid. 
This leaves (1) and (4). Loux's phrasing of the Identity of Indiscernibles shows why he thinks a bundle theorist ought to be committed to it when he states it as, "necessarily if a thing, $x$, and a thing, $y$, have all and only the same properties, then $x$ is identical with $y$." As the bundle trope theorist is committed to non-repeating properties, it then stands that anything with the same properties as $x$ is identical with $x$ : metaphysically, nothing else could share those properties. But I think that this reading of the Identity of Indiscernibles does disservice to the trope theorist. Consider how Black frames his objection to the Identity of Indiscernibles with his radially symmetrical universes example:

Isn't it logically possible that the universe should have contained nothing but two exactly similar spheres? We might suppose that each was made of chemically pure iron, had a diameter of one mile, that they had the same temperature, colour, and so on, and that nothing else existed. Then every quality and relational characteristic of the one would also be a property of the other. Now. if what I am describing is logically possible, it is not impossible for two things to have all their properties in common. ${ }^{191}$

A trope theorist who wished to affirm the Identity of Indiscernibles could deny the thoughtexperiment's conclusion that the two spheres have "all of their properties in common." The tropes of one iron sphere are different tropes than those of the other. As Loux relies on such thought-experiments to put the bundle theorist in a predicament here, his objection may fall flat. ${ }^{192}$ But more charitably to Black, one can note that he is describing 'exactly similar spheres.' And the trope theorist should at least allow a universe which is composed of nothing but two spheres of iron which maximally resemble one another. On this reading, the experiment only affirms the qualitative identity of the spheres: that their properties are maximally similar to one another. This reading would require a reframing of Loux's version

\footnotetext{
${ }^{191}$ Max Black, "The Identity of Indiscernibles," Mind, Vol. 61, No. 242 (Apr. 1952).

192 Loux, "An Exercise in Constituent Ontology."
} 
of the Identity of Indiscernibles to something like the following: necessarily if a thing, $x$, and a thing, $y$, have all and only qualitatively identical properties, then $x$ is identical with $y$. But a trope theorist can easily deny this, allowing for objects with qualitatively identical but not strictly identical properties. So either the thought experiments which make the Identity of Indiscernibles implausible are unproblematically rejected or a modified version of the Identity of Indiscernibles is easily denied by the trope theorist.

(4) is formulated by Loux in the following question:

But how is it that this theory makes the transition from property to thing having the property? ... Confronted with this question, bundle theorists will likely respond that we ... begin with one first-order property, add another first-order property, add still another. What ultimately emerges is an individual having all those firstorder properties. But why should we suppose what results from this agglomeration yields an individual? Why not suppose instead that what results from this agglomeration is just a conjunctive property whose conjuncts are the various first-order properties? ${ }^{193}$

To put it another way, if there is a distinction between abstract particulars and concrete particulars, with the former being properties and the latter being objects, how is it that a bundle of abstract particulars becomes a concrete particular? The heart of this objection is in what it means to be an object, as opposed to a conjunctive property. But constitutive trope theory can answer this objection, as it is not merely the conjunction of tropes that makes an object what it is: it is that said tropes each emerge from the same parts. By emerging from the same parts, the collection is grounded as a concrete object in a manner which is distinguishable from the mere conjunction tropes, much in the same manner as the substratum theories. As Loux shows that this grants substratum theories immunity to this

193 Ibid. 
objection, ${ }^{194}$ so too is constitutive trope theory immune. So as we can see, Loux's problems for the bundle theory do not apply to constitutive trope theory.

\subsubsection{Justifying Commonality}

One final objection may be brought forward, in that constitutive trope theory has not done enough to satisfy our second desideratum: it has not adequately explained the similarity we see in the world. To see how this objection can run, consider the possibility of a world where constitutive trope theory is true, but there is no actual similarity within that world. Foundational tropes in this world do not resemble each other in any way other than their being foundational, and when these dissimilar tropes enter into relations sufficient for some new property's instantiation, the resulting tropes are dissimilar to any other foundational or emergent trope. This world, which lacks any similarity, may be possible given constituent trope theory. ${ }^{195}$ As constitutive trope theory does not rule out such a world, it does not adequately explain similarity.

There are two responses to this objection, I think. The first is that other ontologies also fail to rule out the dissimilar world as possible. The second is that while constitutive trope theory does not itself rule out the dissimilar world, it can add to a contingent explanation of why the dissimilar world is not actual. To see that the dissimilar world is

\footnotetext{
194 Ibid.

195 One might think that such a world is not truly possible, as each trope at this world hold the relational property of 'being dissimilar to any other trope.' As such, these relational properties between tropes will be similar to each other and, as a result, there are some properties at this world which are similar. This objection to the scenario follows through if this relational property is a genuine property: a trope. The objection may still follow through if the world only has two foundational tropes or a single foundational trope and a single emergent trope which emerges from the foundational trope. However, if one finds the scenario of this objection implausible, so much the better for constitutive trope theory.
} 
possible on other theories, let's first turn to realism. For simplicity's sake, imagine a world with only two objects. One of these objects has mass and spin but no charge or velocity ${ }^{196}$. The other has no mass, or spin but a velocity and a charge. Further, imagine that each of these properties is a singly-instantiated universal. Insofar as our first possible world is consistent with constitutive trope theory, this world is consistent both with the dissimilar world and the realist picture. For a resemblance-nominalist world, imagine a world with some number of objects, none of which enter into resemblance relations with the others. This world is as possible for the nominalist picture as our first was for constitutive trope theory. So generally speaking, such a world is not ruled impossible by other theories either. But constitutive trope theory can give us a contingent explanation for why the actual world is not a dissimilar world. According to constitutive trope theory, the relations between constitutive parts that form the structures which ground tropes are spatiotemporal relations and causal relations. This leads to there being commonality in the structures that give rise to properties: they are of a kind. Further, in the actual world, as we have gone into lower-levels of physics we have discovered fewer kinds of objects in each more basic category: from an uncountable number of kinds of molecules to 118 known elements of the periodic table to the 17 sub-atomic particles of the current standard model. ${ }^{197}$ Lastly, at each level we find kinds that are disposed to create new, emergent properties when in the appropriate relations, with some of these being easier to instantiate

\footnotetext{
${ }^{196}$ We might worry that such an object isn't possible for the properties in question. But we need not use these four properties: they are merely inserted to be more illustrative.

197 One may cast doubt here, as there may be undiscovered particles that would call this into jeopardy: dark matter or other discoveries may add more to a layer: there may turn out to be more kinds of sub-atomic particles than atoms, for instance. I don't think this line of speculation is more than that. Trying to determine the number and kinds of entities that exist through speculation alone has not historically worked out.
} 
in natural conditions than others. As an example, helium and hydrogen do not require atomic fusion to be formed (most helium and hydrogen having been formed by Big Bang nucleosynthesis) while more complex particles do. Further, many emergent properties can be instantiated in more than one way. Given that these are the sorts of properties and the sorts of objects we find populating our universe, constitutive trope theory can explain the commonality we find in the world in virtue of the common structures and similarity between elementary kinds that pervades our world. Given that other theories may also allow for the possibility of dissimilar worlds, constitutive trope theory gives us tools to explain why our world shows so much similarity is an advantage for the theory.

\subsection{Placing Within the Debate}

With some potential objections to constitutive trope theory now out of the way, we may attempt to place it within the greater context of ontological debates: both those with respect to tropes and with respect to grounding the truth of laws of nature. Some of this is immediately obvious. Constitutive trope theory is a theory of tropes, as opposed to realist or nominalist accounts of properties. But beyond this simplistic categorization, some distinctions may be unclear and others have yet to be made.

Among trope theories, the primary division in classification is based in the relationship between tropes and their objects. Most trope theories are either bundle theories, which state that objects are nothing over and above a colocation of tropes, and substratum theories, which state that there is a bare particular at the 'core' of the object to which its tropes belong. A few exceptions evade this classification, such as Simon's nuclear theory. Constitutive trope theory is likewise such an exception. While our primary analysis 
of objects is consistent with the bundle theory and bare particulars are not postulated, compresence relations are not taken as a primitive relation of the theory. Instead, compresence between tropes occurs due to relations between lower-level objects that are sufficient for the existence of the tropes. The tropes that result from these relations are compresent because they have the same parts. As such, for non-foundational tropes, the theory admits of substrata as the basis for tropes. So while constitutive trope theory resembles a bundle theory with respect to the relationship between tropes and the objects to which they belong, it resembles a substratum theory in how those tropes are grounded. For these reasons, it neither fits well as a bundle theory nor a substratum theory.

But there are other categorizations that may help further, such as Garcia's division between module and modular tropes. ${ }^{198}$ Garcia divides trope theories along a lines similar to but distinct from the bundle/substratum distinction. Module tropes are what Garcia refers to as "tropes as objects." That is, the tropes are considered entities that exist without relying on some other entity. Modular tropes instead act as modifiers on some other extant entity. An illustration of this distinction may be useful here. Consider 'red' as a property. On module trope theories, a particular case of redness is an entity in its own right. On modular trope theories, a particular case of redness is not an entity in its own right but is an aspect of the nature of another entity such as a bare particular. Garcia makes the case that classic bundle theories of tropes take tropes to be module tropes while classic substratum theories take tropes to be modular tropes. For constitutive trope theory, while the existence of a given trope is grounded in the relations of its parts, the resulting trope does not modify the nature of those parts: the resulting trope is an entity in its own right. And thus, using

\footnotetext{
${ }^{198}$ Loux refers to these as 'tropes' and 'tropers,' respectively.
} 
Garcia's distinction, constitutive trope theory takes tropes to be module tropes. This does open constitutive trope theory to certain objections normally pressed against bundle theories, such as those of Loux, which have already been addressed.

\subsubsection{Number of Properties}

In the metaphysics of properties, one prominent debate that has not been touched on here at much length is with respect to the number of properties that are admitted as genuine. Typically, theories are characterized as either sparse or abundant, although exactly what these terms mean varies slightly from philosopher to philosopher. Both for the sake of clarity and to make further distinctions, I will define the terms in the following way:

Sparse- A sparse theory of properties admits only properties which fit a particular demarcation, such as only the properties of physics or those of ordinary experience.

Abundant- An abundant theory of properties has no particular demarcation criteria but may not admit certain 'problematic' properties, as defined by the theory.

I will also introduce two new distinctions: eliminitivist and profuse theories of properties. They are as follows:

Eliminitivist- An eliminitivist theory of properties admits only (some) foundational properties as genuine properties.

Profuse- A profuse theory of properties admits of all properties into its ontology, including those considered problematic on many other ontologies. 
While this latter distinction is not made in the literature, it is present at points. Armstrong's version of 'sparse' and 'abundant' properties may more neatly fit the eliminitivist vs. profuse distinction here, and Armstrong himself seems to prefer an eliminitivist ontology, saying that the only properties he is truly willing to admit are those of the most fundamental physics, whatever they may be. ${ }^{199}$ Campbell's field theory is also an eliminitivist theory, ultimately declaring that the properties we are familiar with are not tropes but are instead pseudo-tropes which result from variations in the fields he maintains are the true tropes. The abundant vs. profuse distinction also allows us to distinguish between theories which have no particular demarcating feature from those which are committed to the existence of every property.

Making the distinctions this way, constitutive trope theory is clearly neither an eliminitivist nor a profuse theory of properties. It is not eliminitivist as it admits emergent tropes and is not profuse as it does not admit of absences or aggregates as genuine properties. This leaves only whether it is committed to an abundant or a sparse theory of properties. This boils down to whether or not there is a specific demarcating criterion for genuine properties for constitutive trope theory or not.

I believe that while it is good for a holder of constitutive trope theory to accept such a demarcating criterion, it is not necessary that they do so. The clearest demarcating criteria for constitutive trope theory, given the theory of grounding for scientific lawstatements attached to it, is one where only those tropes which have proper dispositions are genuine tropes. Given that dispositions act as the grounding for scientific lawstatements, this would also mean that all tropes are properties for which there can be an

${ }^{199}$ Armstrong, "A Theory of Universals." 
accurate scientific law. But this commitment is not necessary for the constitutive trope theorist. Dispositionless tropes have not been specifically ruled out: there are simply no laws regarding them. If a constitutive trope theorist wishes to admit dispositionless tropes, three things may be said of them. First, there can be no laws regarding them. Second, they cannot be relevant to any relations for emergent tropes, although objects which possess them may still serve as constitutive parts. Third, the existence of any such trope is inherently unverifiable a posteriori and impossible to observe. ${ }^{200}$ This does give Occamist reasons to eliminate them from one's ontology: the ontology is cleaner if all tropes are dispositional rather than if merely some tropes are dispositional. But while I prefer this line of demarcation, if one prefers an abundant ontology to a sparse one then constituent trope theory does not explicitly decide between these two views.

\subsection{Falsification of Constitutive Trope Theory}

There is one final implication of constitutive trope theory that I would like to address: its falsifiability. Unlike many competing theories, constitutive trope theory makes predictions about the world and the kinds of things that one can find in it which allow the theory, at least in principle, to be empirically falsified. I take this to be a further reason, beyond the desiderata given in Chapter 1, to accept constitutive trope theory. As the theory can be empirically falsified, it presents an opportunity for theorists to challenge it in a way that other theories of properties do not.

While the virtues of a theory's being falsifiable are well accepted by philosophers with respect to scientific practice, fewer philosophers admit that theories of metaphysics

200 This was handled in more detail in Chapter 3. 
ought to be falsifiable as well. Before showing how constitutive trope theory can be falsified, I would like to present several arguments against the practice of making one's ontology falsifiable and address them here. The first argument I will consider is that metaphysics deals with necessary truths while science deals with contingent truths. The second is that metaphysics is not akin to science and thus not subject to the same sorts of proofs. The third is that the entities of metaphysics are more fundamental than those of scientific practice. The fourth is that philosophical theories are linguistic or conceptual truths, not suitable for falsification. Lastly, I will consider the argument that falsification is not even the standard for scientific theories in the modern era, much less metaphysical theories.

The first argument against a falsifiable metaphysics goes as follows: the truths of metaphysics are necessary while the truths of science, even fundamental physics, are merely contingent. To use possible worlds talk, we may posit a world with different physics with no contradiction, but not a world with different metaphysics. Consider, for instance, Lewis' possible worlds realism. If it is true, it must be true at every world. If it is false, it must be false at the only world, the actual world. But for falsifiable theories, there is some world at which they are false. As such, presenting metaphysical truths as falsifiable is making a category mistake.

While I agree that there is a difficulty here if one assumes possible world realism, we can instead consider philosophical theories as models for describing events, actual or not. We can compare a realist theory and a nominalist theory, see what predictions they make about the structure of the world, and ask what the differences would be between the worlds each predicts. We can compare an account of possibilia or identity against the actual 
world and ask if the picture the account gives us matches what we observe, or if there are inconsistencies between what the theory predicts and what we observe. This regularly occurs in philosophy already. For instance, one may criticize Lewis' possible worlds realism because we cannot observe other possible worlds and so it is not clear how they can serve as the truthmakers of possibility claims. ${ }^{201}$ Even Lewis himself addresses this criticism. ${ }^{202}$ Regardless of whether or not the criticism fails, this is an attempt to falsify the claim given our observations about the world. It says that Lewis' theory is wrong because it fails to give an account where we can have knowledge of the truth of possibility claims.

Further, if the claims are purported to be necessary truths, I argue this ought to make them more open to falsification, not less. If a claim is not merely true but necessarily true, it follows that it can never be false in the actual world. Further, scientific theories are normally limited by their subject matter: the claims of a scientific theory may only pertain to particles or molecules or animals of a particular kind. A metaphysical theory, on its face, has a much broader domain: all of being. As such, metaphysical theories ought to be more open to falsification precisely because they purport to have a higher level of necessity. Just as if one purports that $2+2=5$, rejecting the claim requires only that I find an instance where 2 and 2 combined additively do not equal 5. For instance, I may provide 2 piles of 2 books and have them count the number of books. But it does not matter if I use books or cups or rocks, the falsification will be the same. Similarly, metaphysical theories should be more open to rejection through falsification than theories of science.

201 Both Richards (1975) and Lycan (1979) bring forward this objection.

${ }^{202}$ Lewis, "On The Plurality of Worlds,": 112. 
Another argument presents itself in the claim that a posteriori investigation is only applicable to the sciences, and that metaphysical entities are more primitive than scientific entities. ${ }^{203}$ This is to say, that the kinds of entities described by the various sciences must also be more broadly handled by a metaphysical theory if the scientific entities are to be genuine objects in the world. With metaphysical entities thus being more primitive than the observed kinds of science, they can only be analyzed a priori. I do not see much merit in this argument. I think that it stems entirely from a desire to keep philosophical theories and scientific theories demarcated from each other. First, it is not clear that being more primitive entails that only a priori investigations will be possible or desirable. Second, even if metaphysical entities are more primitive, the entities of metaphysics are still in the world: the same as entities studied through science. As both metaphysics and science make claims about the external world, a posteriori investigations ought to be able to verify their claims. Lastly, even if philosophical theories are or ought to be distinguished in terms of a priori rather than a posteriori study, this would only indicate that metaphysical theses ought to be taken as scientific. As metaphysical theses are about entities in the world, and entities in the world are capable of being studied a posteriori, this means that the objects of metaphysics are capable of a posteriori study. If the demarcation between science and philosophy is that science is a posteriori and philosophy is a priori, then this would make metaphysical entities the subject of scientific study. If some metaphysical questions cannot undergo this sort of investigation, then perhaps the discipline is split between science and

\footnotetext{
203 One might wonder, rightly, about the distinction between metaphysical entities and scientific entities. This is a fair worry. However, given that I am arguing against the claim that metaphysical proofs cannot be subject to the same empirical investigations as scientific proofs, this is not for me to demarcate. Rather, it is the person wishing to use this distinction who needs to draw this line.
} 
philosophy: but those questions of metaphysics which regard the world and its entities ought then be a part of the sciences. But I don't think we need to go this far, as philosophers check their theories against a posteriori results regularly, and it is not an uncommon criticism of a theory that it implies some answer that is contrary to what has been observed. Take, for instance, this argument advanced by Prior, Pargetter, and Jackson: We cannot say both that being fragile $=$ having molecular bonding a, and that being fragile $=$ having crystalline structure B; because by transitivity we would be led to the manifestly false conclusion that having molecular bonding a = having crystalline structure B. ${ }^{204}$

It is the a posterori fact that having molecular bonding a and crystalline structure B are distinct that grants this argument any weight at all. In fact, if we could find no case where the same disposition is present in multiple kinds of material structures, her argument against this would fail. The theory that they attack is rejected precisely because it is empirically falsified. Philosophers commonly make use of such devices, and so implicitly accept that metaphysical theories may have empirical consequences that are falsifiable.

The third argument is similar to the second. It argues that metaphysical posits are necessary for scientific investigation: that some metaphysics must underlie scientific claims. Given this, the argument goes, metaphysical entities cannot be subject to the same sorts of investigation as scientific ones. I think this argument falls much into the same criticisms as the previous one: if the entities of metaphysics are of the world, it is not clear why they should be immune to a posteriori investigation merely because they underlie the entities of scientific theories. But additionally, it is worth noting that the claim that

${ }^{204}$ Elizabeth W. Prior, Robert Pargetter and Frank Jackson, "Three Theses About Dispositions," American Philosophical Quarterly 19, No. 3 (Jul. 1982). 
scientific investigation requires a particular metaphysics is dubious. Scientists in practice appear to be able to do their investigations just fine without underlying metaphysical posits, just as chemists and biologists seem able to do good work without relying with any strength on the physical sciences which underlie their disciplines, save where the theories of one directly interact with the theories of the other. It does not appear that any investigation into the nature of the world actually requires that we go to whatever is most basic to understand it: merely understanding the objects of the theory and how they are brought about and interact with other objects in the theory is sufficient. Scientists may even disagree about metaphysical truths, but this does not seem to affect their investigations: there are no cases of a Humean about properties finding different empirical results than a realist or any similar result. The claim is made more dubious by the fact that our investigations into the world appear to start at a very surface level of immediate perception and venture into more basic entities from there, rather than starting with the most basic entities and trying to build a science from the bottom up. So the idea that metaphysical posits are necessary for scientific investigation does not match scientific practice either: we start with the observable and move to the theoretical, not the other way around. The mere fact that scientific investigation can continue without answers to deeper metaphysical questions tells us that answers to these questions are not required for empirical research. As such, it is not clear that metaphysical posits are necessary for scientific investigation and there are good reasons to deny this claim.

A fourth critique of a posteriori investigation comes from those who would say that philosophy itself is not, by and large, concerned with truths about the world at all in the manner that science is. Instead, philosophy is concerned primarily with linguistic, 
conceptual, or analytic truths which are not directly about the world. This sort of criticism might come from a logical positivist such as Carnap. Being about linguistic, conceptual, or analytic truths, philosophy is not the sort of thing that is subject to a posteriori analysis. If the premise of this critique is true about the status of philosophy then I would agree. Only I think that such a critique jumps off the boat long before we are at the point of a critique of falsification in metaphysics, as it is hard to see metaphysics as even a tenable discipline at that point. Metaphysical claims are claims about the world, so if philosophy is not in the business of making claims about the world, then metaphysics either does not belong to philosophy or should be eliminated entirely as a discipline. Indeed, philosophers such as Carnap would generally want to do away with metaphysical talk entirely. I tried to show in Chapter 1 why I think this work needs to be done and, if I have succeeded, then I do not think it requires a second defense here. But if metaphysics is a discipline worthy of study at all, its posits are not merely linguistic, conceptual, or analytic.

One final critique is not against a posteriori investigation in metaphysics, but that falsification is the wrong track on which to do it. There are two versions of this criticism. The first comes from a Kuhnian direction, which advocates that science is advanced in terms of paradigms and not falsification, that while small-scale examples of theory falsification can occur within a scientific paradigm, it is ultimately the shifting of paradigms that leads to change in science. Another is that more sophisticated methods, such as Bayesian inference methods, have overtaken falsification as the standard for a posteriori investigation. For the former criticism I would argue that while falsification may not be sufficient for a paradigm shift that it can be a step in it. Even Kuhn advocated that data 
which falsifies a set theory is a step in the process of a paradigm shift. ${ }^{205}$ Paradigm shifts are, in part, motivated by the existence of data which currently accepted theories do not adequately explain. This indicates either that the theory is incomplete, or that the data contradicts some aspect of the theory. The former case allows for mere modification of the theory and need not necessitate a paradigm shift provided that the additions to the theory are compatible with the theory as it stands. If such modifications are incompatible with the theory or the data contradicts the theory outright, we have cases of falsification. If this is so, the ability of the theory to generate predictions that can in-principle be falsified is a step prior to this: an unfalsifiable theory cannot generate such predictions. To the Bayesian, I would point out that confirmation of constitutive trope theory is possible, and perhaps even trivial: it exists in every case of successful scientific explanation where a property's existence and character is explained through the relations of the parts of its object. This combined with falsification allows for the theory to be given a standard likelihood ratio which allows for a Bayesian analysis. But the falsifying instances are, I think, both more damning for the theory if they exist and more unusual for a metaphysical theory. And so I include them here.

Having addressed some criticisms of the method, I would like to say one more thing before going into the ways the theory might be falsified. Some of the methods suggested may seem impossible to a reader, either in a physical or metaphysical sense. I will endeavor to show that they are not, although they might seem implausible. If they seem implausible to the reader then that only shows that the theory matches your common sense. This may be a mark for the theory, but I would hesitate to consider it a strong mark in favor of it: a

205 Thomas Kuhn, The Structure of Scientific Revolutions, (Chicago, University of Chicago Press, 1970). 
good many things that are actual may seem implausible, as theoretical physics

demonstrates. The fact that the theory offers many paths to falsification that will be explored but none which are fulfilled is a much stronger argument in its favor.

\subsubsection{Falsifying the Constitutive}

The first place where constitutive trope theory may be falsified in its analysis of constitution and how parts are related to tropes. Here, I will demonstrate at least some of the ways that constitutive trope theory can be falsified on these grounds. One way would be through part-independent emergent tropes. Another would be through part-identical emergent tropes. A third is through part-removed emergent tropes. A fourth would be through non-objects acting as constituents of the theory. These methods of falsification center on there being certain sorts of trope that exist in the world, and so one looking to question constituent trope theory's commitment to falsificationism might claim that the constituent trope theorist can simply deny that anything that meets these criteria is a trope. However, such denials would only call the theory into question, particularly if the theory requires that we eliminate properties which are useful to scientific inquiry or which otherwise fit the description of tropes on this theory.

By a part-independent emergent trope, I mean an emergent trope which exists apart from a supervenience base. This may seem implausible on its face: if the trope has a supervenience base, then how can it be independent of it? But we can imagine parts acting as 'trope generators' yet not 'trope sustainers': where tropes were generated by their underlying parts but required no parts to sustain them, operating freely once brought into the world. This is different than the tropes finding some new supervenience base, as can 
happen in cases of overdetermination. Instead, such a trope would no longer need a supervenience base at all, floating freely once generated. If a number of such tropes were generated together and maintained cohesion, they could even form a particular object. If any such tropes existed, it would call into question the entire case for emergent tropes requiring some supervenience base for their existence: a devastating blow for the theory. Part-identical emergent tropes would be trickier and may require a more global rejection of the metaphysics. But straightforwardly, if tropes were identical to their supervenience base ${ }^{206}$ the theory would fall apart. Showing how this rejection would work may help us understand it. First, one would have to show that in cases of overdetermination, multiple tropes actually exist. Take a case where what is currently thought to be one trope has three parts, A, B, and C, where any two of these parts would be sufficient for the existence of the trope in question. If in these cases it could be shown that there were three distinct, overlapping tropes and not one overdetermined trope then this would be a significant blow to constitutive trope theory. But in addition, you would need to show that having a different kind of supervenience base resulted in a different kind of trope. This is because the parts themselves are different. The tropes A and B and the tropes $\mathrm{B}$ and $\mathrm{C}$ are non-identical: they are distinct sets of parts. Thus, if the trope is to be identical with its supervenience base, the previously thought to be overdetermined tropes that arise from A, B, and C must also be different tropes. This would mean that cases currently thought to be cases of overdetermination would need a way to be properly distinguished.

\footnotetext{
206 Of course, if they are identical it is not actually a supervenience base as the collective parts are identical to the trope(s) in question. However, adequately describing the distinction is difficult and would require switching between the two paradigms at once. As such, I refer to the parts and their relations as a supervenience base in either case for the sake of clarity.
} 
Part-removed emergent tropes would be tropes which have a supervenience base and, unlike part-independent emergent tropes, do rely on their parts for their continued existence. However, these tropes are spatiotemporally distinct from their parts: they exist in a different region of spacetime. So a collection of atoms here may result in a property being instantiated in another point in space or time. ${ }^{207}$ As spatiotemporal location is derived from parts, at least down to a level where spatiotemporal properties exist, constitutive trope theory is committed to their location being identical. Unlike other cases, this one may not falsify the theory as a whole: only the theory's conclusions about spatiotemporal properties would need to be adjusted. But it would require significant modifications to the theory, and those consequences could be significant enough to call into question the derivation of other properties from their parts which could prove more devastating.

Finally, we can take the case of non-objects acting as parts in the theory. This is different than pseudo-objects such as aggregates acting as parts. Our clearest example of a non-object on this theory would be an absence. For simplicity, imagine an object which consists of three object-parts, A, B, and C, and relations $r 1(A B), r 2(B C), r 3(A C)$, and r4(A\{\}) where \{\} represents an absence. So in addition to $A, B$, and $C$ being related to each other in particular ways, A must also be related to an absence for the resulting tropes of the object to exist. This would either call into question that objects are the proper parts of a theory or

\footnotetext{
${ }^{207}$ An interesting case is that of quantum entanglement, where changes to one particle may impact a particle that is spatiotemporally removed without any communication between them. This does not qualify as a counter-example, however, as the entangled particles are not constitutive parts of each other. It is more accurate to analyze entanglement as a relation between particles or as a single object which is composed of the entangled particles.
} 
that non-entities are not objects. In either case, it would be disastrous for the picture of emergent tropes given here.

\subsubsection{Falsifying via Foundations}

Foundational entities have perhaps had the fewest things said about them of anything in the theory. Nevertheless, there are several ways that foundational entities could turn out to be that would call the theory into question. The weakest of these is if foundational entities turned out to be multi-charactered. Another would be for foundational entities to be multiply present. A third would be for foundational entities to be uncharactered. A final way would be for foundational entities to be absences. Unlike some of the other ways of falsifying constitutive trope theory, this might be a long time coming: we would first need to definitively find foundational entities. After finding them, proving them to have a particular character and proving that they really are the bottom-most level would take work in the sciences. But once found, any of these might offer a way to falsify constitutive trope theory.

The weakest of these would be if foundational entities are multi-trope objects. Constitutive trope theory offers us an explanation of compresent tropes in terms of their substrata. For foundational tropes, this explanation would not suffice as they have no substrata. This would require invoking something like a primitive compresence relation, which would make constitutive trope theory, at least at the most foundational level, no different than other bundle theories and subject to many criticisms that it has so-far avoided. A caveat here is if the tropes existing compresently can be shown to be physically necessary there is no problem for constitutive trope theory. In other words, if given the 
kinds of properties they are, the most foundational objects must have the properties that they do, that it would be impossible to have foundational tropes of that kind exist without the other foundational tropes they are compresent with, then constitutive trope theory can use this necessity to get out of the problem. If they must necessarily exist together, then this can explain their compresence in a way that is unique to foundational tropes but which does not invoke the problems of a basic compresence relation and as such presents fewer problems for constitutive trope theory. More would have to be said to defend constitutive trope theory, but without specifics as to the foundational tropes and why it is that their natures preclude them from existing without one another, such an account cannot be advanced.

A bigger problem would be if foundational entities are multiply instantiated. This would, in effect, mean that reality at the foundational level consists of universals. While the story of emergence in constitutive trope theory might still be useful, the entire ontology may well need to be replaced with a realist ontology at this point, unless it could simultaneously be shown that non-foundational entities are not multiply instantiated. In any case, any a posteriori proof of this would be devastating for the theory. One might think that such a proof would be impossible. However, imagine that it turned out that foundational objects could be destroyed, but that by destroying one you destroyed all others of the same kind in disparate locations across space-time, or that any change in the one results in all others of that kind changing. If there was no apparent mechanism for this change, but rather it appeared that these disparate entities were in fact changing because they were the same entity, this would effectively show that the property is multiply instantiated in the absence of some other mechanism for that change. 
Similarly, an uncharactered foundational entity, a sort of bare particular with no tropes attached, would prove problematic for constitutive trope theory if discovered to be at the foundations of existence. Imagine such entities as uncharactered points that lack any properties themselves but which possess external relations between them. These entities, lacking any inherent properties, would be problematic for constitutive trope theory as it would force one to admit the existence of objects without tropes, calling the whole enterprise into question.

A perhaps more disturbing possibility is if below the level of emergent tropes there was simply nothing. That is, if it turned out at the layer closest to the foundational, emergent tropes supervened on an absence. This may seem wildly implausible, but I can think of no proof that it is impossible. Here is an argument that might work in favor of it. As we go down in levels of supervenience, we've found that the objects we find are simultaneously more alien to our understanding, particularly at the quantum level, and that they are simpler, with fewer and fewer properties. Thus, objects at the most basic level are likely to have the fewest number of properties. The fewest properties something can have is none. There may be uncharactered objects: bare particulars of some kind. But even an uncharactered object, if one can exist, is still an object. It is simpler still for an absence to exist. Thus at the most basic level, all of the properties may supervene on an absence. I do not think this argument is particularly convincing: I am only trying to make the idea that reality rests on an absence as plausible as possible. And if it does, it would call into question the assumptions of constitutive trope theory. 


\subsubsection{Falsifying via Dispositions}

The relationship between dispositions and tropes is one last place the account may be falsified. This will not have so much to do with the presence or absence of dispositions as with their locus. Constitutive trope theory, taking tropes as its most basic entities, posits that dispositions are not independent entities but rather are qualities of a trope. Accordingly, constitutive trope theory could be falsified by either the existence of tropefree dispositions or trope-independent dispositions. A trope-free disposition is just that: a disposition without an accompanying trope. A trope-independent disposition is akin to a part-independent trope: it is brought into existence by a trope, but once brought into existence may exist apart from that trope.

To talk of trope-free dispositions, we do not need to use absences as our example case. Aggregates will do fine and may seem more plausible. If a true aggregate, a collection of objects which does not result in any emergent property, could have a disposition that is not merely the collective dispositions of the objects constituting the aggregate, it would show that constitutive trope theory is incorrect. ${ }^{208}$ This may seem paradoxical: if the collective objects of the aggregate result in a disposition, wouldn't that disposition be a property? Perhaps not. Imagine that it turns out that if you get exactly 5,400 grains of sand within a 3" radius they all begin to move east at a rate of $1 \mathrm{mph}$, regardless of how they are arranged otherwise. Further, they do this as a group and not due to the motion of any particular grain: they move as a unit or not at all. And it is not a matter of 5,400 grains allowing for some lower-level force to push through: 5,401 grains do not get the motion

\footnotetext{
${ }^{208}$ For an example of a collective disposition, consider previous cases examined, such as that of the snake's coloration examined in Chapter 4.
} 
going. It is, to all appearances, merely a brute fact about this particular amount of sand. This propensity to move east would be mysterious and would certainly count as a disposition. But I do not see a way for it to count as a trope: there appears to be nothing about the sand that causes the motion other than the mere presence of enough grains. The behavior emerges from the aggregate, but no property facilitates it. If true, this would violate the grounding of dispositions in tropes, allowing them to be grounded in aggregates as well.

We can also imagine trope-independent dispositions. Imagine an object consisting of two tropes, A and B. Now imagine that the parts sufficient for A and B change such that where $A$ and $B$ are still instantiated, but now a new trope $C$ is also instantiated. Let's stipulate that $\mathrm{C}$ has disposition $\mathrm{d}(\mathrm{C})$. Now imagine that the parts sufficient for $\mathrm{A}, \mathrm{B}$, and $\mathrm{C}$ change again so as to eliminate $\mathrm{C}$. Yet in this, $\mathrm{d}(\mathrm{C})$ survives despite the elimination of $\mathrm{C}$. Further, let's stipulate that neither A nor B have disposition $\mathrm{d}(\mathrm{C})$ nor any disposition like it, and their object had no disposition like $\mathrm{d}(\mathrm{C})$ prior to the arrangement that allowed for $\mathrm{C}$. If such a trope were to exist, it would call into question the premise that dispositions are located as qualities of the trope, as the disposition could apparently continue to exist after the trope that brought it into being ceased to exist. Such a case would violate the theory in question, calling into question the grounding of laws of nature in the dispositional character of tropes.

The ten ways given in this section to falsify constitutive trope theory may not prove the full number, but that was not the goal. The goal was to illustrate a number of ways in which the theory can be falsified. One might worry that the methods given are too abstract or general to be put into practice. But it is precisely because they are abstract and general that they work so well as falsification methods. Constitutive trope theory does not purport 
to only be about a yet undiscovered foundational level of physics: it claims to be of properties from the most foundational physics all the way up to properties of chemistry, biology, psychology, and any other discovered by science. As such, it may be falsified at any of these levels, although the methods utilizing foundational properties must obviously wait until such properties are discovered.

\subsection{Conclusions}

We began this investigation with five desiderata. They were:

1) Answer what it is for an object to have a property;

2) Answer how it is that two different objects can have similar properties, that is, the problem of universals;

3) Give an ontological basis for scientific laws;

4) Assume only the minimal number of theoretical entities needed to provide an account; and

5) Do so with the least amount of unsolved philosophical baggage

I believe I have shown how constitutive trope theory does well with respect to all five criteria in question. It answers the questions in the first three desiderata, only postulates one kind of entity, and does so with little unsolved philosophical baggage. But in addition to these original criteria, I would like to add a sixth now that was not added previously as no other account I know of has met it:

6) Have clear methods of falsification if the theory does not correspond to the actual world. 
And unlike other theories, constitutive trope theory also meets this final desideratum. Other theories fail: either by purporting to be concerned only with foundational entities that are as-yet undiscovered or by not making clear predictions about the relations between properties that are observed in the actual world. Constitutive trope theory does these things, allowing it to be falsified. As such, the theory 'sticks its neck out' in a way that, should it survive, proves it to be a better alternative than competing theories of properties. Of course, this final criteria may backfire: in exposing the theory to the possibility of empirical falsification, I have opened the door for an opponent to discredit the theory quickly should a counter example be forthcoming. I welcome this result, as I believe such a counter example could only serve to increase our knowledge of the world and bring us closer to solving problems in metaphysics. 


\section{Bibliography}

Armstrong, D.M. A Theory of Universals: Volume 2: Universals and Scientific Realism. Cambridge University Press, 1978.

Armstrong, D.M. "A World of States of Affairs." The Journal of Philosophy, vol. 95, no. 10, 1998, p. 535.

Armstrong, D.M. “Against Ostrich Nominalism." Pacific Philosophical Quarterly, vol. 61, 1980.

Armstrong, D.M. "Four Disputes About Properties." Synthese, vol. 144, no. 3, 2005, pp. 309-320.

Armstrong, D.M. Nominalism and Realism: Universals and Scientific Realism, Volume 1. Cambridge University Press, 1980.

Armstrong, D.M. "Reply to Fales." in Ontology, Causality, and Mind: Essays in Honour of D.M. Armstrong. Edited by John Bacon, Keith Campbell, and Lloyd Reinhardt. Cabridge University Press, 1993.

Armstrong, D.M. Universals: An Opinionated Introduction. Westview Press, 1989.

Armstrong, D.M. Nominalism and Realism. Cambridge Univ. Press, 2009.

Armstrong, D.M., et al. Dispositions: A Debate. Routledge, 1996.

Armstrong, D.M. What is a Law of Nature? Cambridge University Press, 1983.

Bacon, John. "A Single Primitive Trope Relation." Journal of Philosophical Logic, vol. 18, no. 2, 1989.

Barker, S., and B. Smart. "The Ultimate Argument Against Dispositional Monist Accounts of Laws." Analysis, vol. 72, no. 4, 2012, pp. 714-722.

Bird, Alexander. "Dispositions and Antidotes." The Philosophical Quarterly, vol. 48, no. 191, April 1998. 
Bird, Alexander. Nature's Metaphysics: Laws and Properties. Oxford University Press, 2009.

Bird, Alexander. "The Dispositionalist Conception of Laws." Foundations of Science, vol. 10, no. 4, 2005, pp. 353-370.

Black, Max. “The Identity of Indiscernibles." Mind, vol. 61, no. 242, April 1952.

Broad, C.D. Scientific Thought. Harcourt, Brace, \& Company Inc., 1923.

Burke, Micheal, "Preserving the Principle of One Object to a Place: A Novel Account of the Relations Among Objects, Sorts, Sortals, and Persistence Conditions." Philosophy and Phenomenological Research, vol. 54, no. 3, Sep. 1994, pp. 591-624.

Campbell, Keith. Abstract Particulars. Basil Blackwell, 1990.

Caplan, Ben, and Bob Bright. "Fusions and Ordinary Physical Objects." Philosophical Studies, vol. 125, no. 1, 2005, pp. 61-83.

Cartwright, Nancy. Hunting Causes and Using Them: Approaches in Philosophy and Economics. Cambridge Univ. Press, 2007.

Cartwright, Nancy. How the Laws of Physics Lie. Oxford University Press, 1983.

Correia, Fabrice. Metaphysical Grounding: Understanding the Structure of Reality. Cambridge University Press, 2012.

Dretske, Fred I. "Laws of Nature.” Readings On Laws Of Nature, pp. 16-37.

Ellis, Brian. Scientific Essentialism. Cambridge University Press, 2007.

Fales, Evan. "Are Causal Laws Contingent?" in Ontology, Causality, and Mind: Essays in Honour of D.M. Armstrong. Edited by John Bacon, Keith Campbell, and Lloyd Reinhardt. Cabridge University Press, 1993.

Fales, Evan. Causation and Universals. Routledge, 1990.

Fuhrmann, Andre. “Tropes and Laws." Philosophical Studies, vol. 63, 1991, pp. 57-82. 
Galluzzo, Gabriele, and Michael J. Loux. The Problem of Universals in Contemporary Philosophy. Cambridge University Press, 2015.

Garcia, Robert K. "Tropes and Dependency Profiles: Problems for the Nuclear Theory of Substance." American Philosophical Quarterly, vol. 51, no. 2, 2014, pp. 167-176.

Hendry, Robin and Rowbottom, Darrell. "Dispositional Essentialism and the Grounding of Laws." Analysis, vol. 69, no. 4, October 2009.

Hintikka, Jaakko. Rudolf Carnap, Logical Empiricist: Materials and Perspectives. D. Reidel Publishing Company, 1975.

Huemer, Michael. "When is Parsimony a Virtue?” Philosophical Quarterly, vol. 59, no. 203, April 2014.

Hume, David. A Treatise on Human Nature. Reprinted by HarperTouch, 2014.

Hume, David. An Enquiry Concerning Human Understanding. Edited by L.A. Selby-Bigge, 2nd edition. Clarendon Press, 1902.

Jaag, Siegfried. "Dispositional Essentialisim and the Grounding of Natural Modality." Philosophers Imprint, 2014.

Kaldor, Nicholas. "A Model of Economic Growth.” The Economic Journal, vol. 67, no. 68, 1957.

Kaplan, David. "Significance and Analyticity: A Comment on Some Recent Proposals of Carnap," in Rudolf Carnap, Logical Emiricist: Materials and Perspectives, Edited by Jaakko Hintikka. Dordrecht, 1975.

Lewis, David. "Against Structural Universals." Australasian Journal of Philosophy, vol. 64, no. 1, March 1986, pp. 25-46.

Lewis, David. Counterfactuals. B. Blackwell, 1973.

Lewis, David. "Humean Supervenience Debugged." Mind, Vol. 103, No. 412, October 1994, pp. 473-490.

Lewis, David. On the Plurality of Worlds. Oxford, Basil, Blackwell, 1986. 
Lewis, David. "New Work for a Theory of Universals." Australasian Journal of Philosophy, vol. 61, no. 4, December 1983.

Locke, John. An Essay Concerning Human Understanding. Ed. by Kenneth P. Winkler, Hackett Publishing Inc., 1996.

Loux, Michael. "An Exercise in Constituent Ontology, in The Problem of Universals in Contemporary Philosophy, Edited by Gabrielle Galluzzo and Michael Loux. Cambridge University Press, 2015.

Lowe, E.J. The Four-Category Ontology: A Metaphysical Foundation for Natural Science. Clarendon Press, 2005.

Lycan, William. "The Trouble with Possible Worlds," in The Possible and the Actual, Edited by Michael Loux. Cornell University Press, 1979.

Martin, C. B. "Dispositions and Conditionals." The Philosophical Quarterly, vol. 44, no. 174,1994

Martin, C. B. “Identity and Exact Similarity." Analysis, vol. 18, no. 4, 1958, p. 83.

Martin, C.B. "The Need for Ontology: Some Choices." Philosophy, vol. 68, no. 266, Oct. 1993, pp. 502-522.

Martin, C.B. "On the Need for Properties: The Road to Pythagoreanism and Back." Synthese, vol. 112, no. 2, Aug. 1997, pp. 193-231.

Martin, C.B. "Properties and Dispositions," in Dispositions, Edited by Tim Crane. Routledge, 1996.

Martin, C.B. "Substance Substantiated." Australasian Journal of Philosophy, vol. 58, no. 1, 1980, pp. 3-10.

Maurin, Anna-Sofia. "Trope Theory and the Bradley Regress." Synthese, vol. 175, no. 3, Jan. 2009, pp. 311-326.

Maurin, Anna-Sofia. If tropes. Springer, 2002.

Mellor, D. H, and Alex Oliver. Properties. Oxford University Pres, 2002. 
Mumford, Stephen. Laws in Nature. Routledge, 2002.

Mumford, Stephen, and Anjum, Rani Lill. Getting Causes From Powers. Oxford University Press, 2014.

Nolan, David. "Quantitative Parsimony." The British Journal for the Philosophy of Science, vol. 48, no. 3, September 1997.

Price, Henry Habberley. Thinking and Experience. Hutchinson University Library, 1953.

Prior, Elizabeth, Pargetter, Robert, and Jackson, Frank. "Three Theses About Dispositions." American Philosophical Quarterly, vol. 19, no. 3, July 1982.

Quine, W. V. "Soft Impeachment Disowned." Pacific Philosophical Quarterly, vol. 61, no. 4, 1980, pp. 450-451.

Quine, Willard V. "On What There Is." The Review of Metaphysics, vol. 2, no. 5, Sept. 1948, pp. 21-38.

Ramsey, Frank Plumpton. The Foundations of Mathematics and Other Logical Essays. Humanities Press, 1931.

Russell, Bertrand. Why I am not a Christian and Other Essays on Religion and Related Topics. George Allen \& Unwin Ltd, 1957.

Richards, Tom. "The Worlds of David Lewis." Australasian Journal of Philosophy, vol. 53, no. 2, 1975. pp. $105-118$.

Schaffer, Jonathan. "Monism: The Priority of the Whole." Philosophical Review, vol. 119, no. $1,2010$.

Simons, Peter. "Identity Through Time and Trope Bundles," Topoi, vol. 19, 2000, pp. 147-155.

Simons, Peter. "Particulars in Particular Clothing," Philosophy and Phenomenological Research, vol. 54, no. 3, 1994.

Sider, Theodore. Writing the Book of the World. Clarendon Press, 2013. 
Sober, Eliot. "The Principle of Parsimony." The British Journal for the Philosophy of Science, vol. 32, no. 2, June 1981.

Sosa, Ernest. Causation and Conditionals. Oxford University Press, 2004.

Stout, George Frederick. Mind \& Matter. Cambridge University Press, 1931.

Swoyer, Chris. "THe Nature of Natural Laws." Australasian Journal of Philosophy, vol. 60, no. $3,1982$.

Tahko, Tuomas, “Boring Infinite Descent.” Metaphilosophy, vol. 45, no. 2, April 2014.

Tooley, Michael. "The Nature of Laws." Canadian Journal of Philosophy, vol. 7, no. 4, Dec. 1977, pp. 667-698.

Van Frassen, Bas. Laws and Symmetry. Oxford, 1989.

Williams, D.C. The Ground of Induction. Harvard University Press, 1947.

Zimmerman, Dean W. “Could Extended Objects Be Made Out of Simple Parts? An Argument for "Atomless Gunk"." Philosophy and Phenomenological Research, vol. 56, no. 1, March 1996. 